\title{
Access to Diverse Oxygen Heterocycles via Oxidative Rearrangement of Benzylic Tertiary Alcohols
}

\author{
Brandon T. Kelley, Jennifer C. Walters, Sarah E. Wengryniuk* \\ Department of Chemistry, Temple University 1901 N. $13^{\text {th }}$ Street, Philadelphia, Pennsylvania 19122, United States \\ sarahw@temple.edu
}

\section{Supporting Information}

Table of contents

Instrumentation and General Notes

Page

General Procedure for Tertiary Alcohol Synthesis and Experimental Data (7, S-20 - S-30)

$\mathrm{SI2}$

General Procedure for Oxidative Rearrangement and Experimental Data

SI2-6

Experimental data for $35-3813,18$, and 19

SI6-10

Table of Failed Reagents

SI10-12

${ }^{1} \mathrm{H}$ NMR and ${ }^{13} \mathrm{C}$ NMR (APT) Data of all Unknown Compounds

SI13

Crystal Structure Report for Acetal 22

SI14-73

Crystal Structure Report for HVI 18

SI74-79

References

SI80-86

SI12 


\section{General Information:}

${ }^{1} \mathrm{H}$ and ${ }^{13} \mathrm{C}$ NMR spectra were recorded at $500 \mathrm{MHz}$ and $125 \mathrm{MHz}$ on a Bruker Advance 500 or $400 \mathrm{MHz}$ and $100 \mathrm{MHz}$ on a Bruker Advance 400. ${ }^{1} \mathrm{H}$ NMR chemical shifts were reported in part per million (ppm) from the solvent resonance $\left(\mathrm{CDCl}_{3}\right.$ $7.26 \mathrm{ppm}$ ). The data was reported as follows: chemical shift number, multiplicity $(\mathrm{s}=$ singlet, $\mathrm{d}=\mathrm{doublet}, \mathrm{t}=$ triplet, sept $=$ septet, $d d=$ doublet of doublets, $t d=$ triplet of doublets, $m=$ multiplet. Proton decoupled attached proton test (APT) ${ }^{13} \mathrm{C}$ NMR shifts were reported in ppm from the solvent resonance $\left(\mathrm{CDCl}_{3} 77.0 \mathrm{ppm}\right)$. The reaction solvents used were anhydrous (HPLC-grade solvent passed through an activated-alumina column). HFIP was purchased from Oakwood Chemicals, distilled over $\mathrm{CaH}_{2}$ and stored over activated $3 \AA$ molecular sieves under an atmosphere of argon. All other reagents were used without further purification. [( $\left.\left.\mathrm{Py}^{+}\right)_{2} \mathrm{IPh}\right] 2 \mathrm{OTf}$ was synthesized and used without further purification. Flash chromatography was carried out using Sorbent Technologies silica gel $60 \AA(40-63 \mu \mathrm{m})$ in the solvent system listed in the individual experiments. The reactions were monitored using analytical thin-layer chromatography (TLC) on Merck silica gel (60 $\mathrm{F}_{254}$ ) plates. GCMS analysis, was done using an Aglient 7980B GC and a 5977A MS. The products were separated on a crossbond $5 \%$ diphenyl-95\% dimethyl polysiloxane column (30 m length by $0.25 \mathrm{~mm}$ i.d., $0.25 \mu \mathrm{m}$ df). Helium was employed as the carrier gas, with a constant column flow of $1.2 \mathrm{~mL} / \mathrm{min}$. The injector temperature was held constant at 250 ${ }^{\circ} \mathrm{C}$. The GC oven temperature program was as follows: $40^{\circ} \mathrm{C}$, hold for $1 \mathrm{~min}$, ramp $25^{\circ} \mathrm{C} / \mathrm{min}$ to $300{ }^{\circ} \mathrm{C}$, and hold for 2 mins. Accurate mass spectra were recorded on an Agilent 6520 Accurate-Mass Q-TOF LC/MS. Infrared spectra were obtained using a Thermo Nicolet Nexus 670 FTIR spectrophotometer. Melting points are uncorrected.

\section{General Procedure for Synthesis of Tertiary Alcohols:}

The ketone ( 1 equiv.) was added to a flame-dried flask, under argon, and was dissolved in dry THF (0.2 M). The solution was cooled to $0{ }^{\circ} \mathrm{C}$ and the appropriate Grignard was added to the solution (2.5 equiv) and the solution was slowly warmed to room temperature, monitored by TLC and allowed to stir overnight. Upon completion, the reaction was cooled to $0{ }^{\circ} \mathrm{C}$ and quenched with sat. $\mathrm{NH}_{4} \mathrm{Cl}$. The partition was warmed to room temperature and the two layers were separated. The aqueous layer was extracted twice with diethyl ether and the combined organic layers were dried over $\mathrm{Na}_{2} \mathrm{SO}_{4}$, filtered and concentrated. The crude alcohols were purified by flash chromatography to give the desired $3^{\circ}$-alcohol.

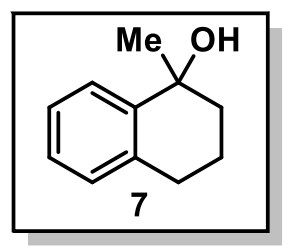

1-methyl-1,2,3,4-tetrahydronaphthalen-1-ol (7). The general procedure was followed to give the product as a white solid (3.03 $\mathrm{g}, 62 \%)$. Spectral data is identical to that previously reported. ${ }^{1}{ }^{1} \mathbf{H}$ NMR $\left(500 \mathrm{MHz}, \mathrm{CDCl}_{3}\right) \delta 7.60(\mathrm{dd}, J=7.8,1.1 \mathrm{~Hz}, 1 \mathrm{H}), 7.25-7.20(\mathrm{~m}, 1 \mathrm{H}), 7.17(\mathrm{td}, J=7.4,1.5 \mathrm{~Hz}, 1 \mathrm{H})$, $7.10-7.05(\mathrm{~m}, 1 \mathrm{H}), 2.87-2.72(\mathrm{~m}, 2 \mathrm{H}), 2.01-1.89(\mathrm{~m}, 3 \mathrm{H}), 1.88-1.78(\mathrm{~m}, 1 \mathrm{H}), 1.77(\mathrm{~s}, 1 \mathrm{H}), 1.57$ $(\mathrm{s}, 3 \mathrm{H})$.

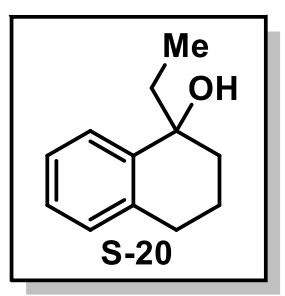

1-ethyl-1,2,3,4-tetrahydronaphthalen-1-ol (S-20). The general procedure was followed to give the product as a white solid $(2.04 \mathrm{~g}, 29 \%)$. Spectral data is identical to that previously reported. ${ }^{2}{ }^{1} \mathbf{H}$ NMR $\left(500 \mathrm{MHz}, \mathrm{CDCl}_{3}\right) \delta 7.53(\mathrm{dd}, J=7.8,1.3 \mathrm{~Hz}, 1 \mathrm{H}), 7.24-7.19(\mathrm{~m}, 1 \mathrm{H}), 7.17(\mathrm{td}, J=7.4,1.5 \mathrm{~Hz}, 1 \mathrm{H})$, 7.09 (dd, $J=7.5,0.9 \mathrm{~Hz}, 1 \mathrm{H}), 2.87-2.70(\mathrm{~m}, 2 \mathrm{H}), 2.08-1.98(\mathrm{~m}, 1 \mathrm{H}), 1.94-1.77(\mathrm{~m}, 6 \mathrm{H}), 1.75(\mathrm{~s}$, $1 \mathrm{H}), 0.90$ (t, $J=7.5 \mathrm{~Hz}, 3 \mathrm{H}$ ). 


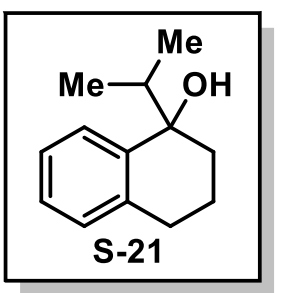

1-isopropyl-1,2,3,4-tetrahydronaphthalen-1-ol (S-21). A modified version of the known procedure was followed. $\mathrm{CeCl}_{3} \cdot 7 \mathrm{H}_{2} \mathrm{O}(3.7 \mathrm{~g}, 10.0 \mathrm{mmol})$ was placed in a flame-dried round bottom flask under argon. The flask was placed in an oil bath and heated to $85^{\circ} \mathrm{C}$ under vacuum overnight. The next day, the powder was heated to $140{ }^{\circ} \mathrm{C}$ for $1 \mathrm{~h}$. While still hot, the flask was back filled with argon, placed in an ice bath, and suspended in THF $(20 \mathrm{~mL})$. The suspension was slowly warmed to room temperature and stirred overnight. The following day, the suspension was cooled to $0^{\circ} \mathrm{C}$ and isopropylmagnesium chloride $\left(5.0 \mathrm{~mL}, 10.0 \mathrm{mmol} ; 2.0 \mathrm{M}\right.$ in THF) was added to the suspension and stirred for $1.5 \mathrm{~h}$ at $0{ }^{\circ} \mathrm{C}$. Next, $\alpha$-tetralone $(665 \mu \mathrm{L}, 5.0 \mathrm{mmol})$ was added to the suspension and the reaction was stirred at $0{ }^{\circ} \mathrm{C}$ and monitored by TLC. Once complete, the reaction was quenched with $10 \% \mathrm{AcOH}(15 \mathrm{~mL})$ and the two layers were separated. The aqueous layer was extracted with EtOAc (2x $15 \mathrm{~mL})$. The combined organic layers were dried over $\mathrm{Na}_{2} \mathrm{SO}_{4}$, filtered, and concentrated. The crude product was purified by flash chromatography to give the desired product as a colorless oil (655 $\mathrm{mg}, 69 \%)$. Spectral data is identical to that previously. ${ }^{3} \mathrm{H}$ NMR $\left(400 \mathrm{MHz}, \mathrm{CDCl}_{3}\right) \delta 7.45(\mathrm{dd}, J=7.8,1.4 \mathrm{~Hz}, 1 \mathrm{H}), 7.17-7.11(\mathrm{~m}, 1 \mathrm{H}), 7.09(\mathrm{td}, J=7.4,1.5$ $\mathrm{Hz}, 1 \mathrm{H}), 7.03-6.99(\mathrm{~m}, 1 \mathrm{H}), 2.74-2.64(\mathrm{~m}, 1 \mathrm{H}), 2.64-2.53(\mathrm{~m}, 1 \mathrm{H}), 2.33($ sept, $J=6.8 \mathrm{~Hz}, 1 \mathrm{H}), 1.85-1.62(\mathrm{~m}, 5 \mathrm{H})$, $1.02(\mathrm{~d}, J=6.8 \mathrm{~Hz}, 3 \mathrm{H}), 0.58(\mathrm{~d}, J=6.9 \mathrm{~Hz}, 3 \mathrm{H})$.
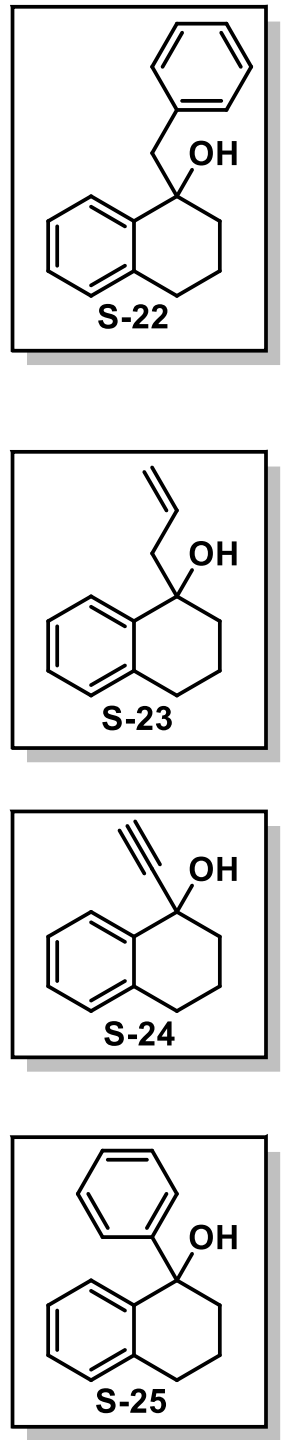

1-benzyl-1,2,3,4-tetrahydronaphthalen-1-ol (S-22). The general procedure was followed to give the product as a colorless oil $(870 \mathrm{mg}, 73 \%)$. Spectral data is identical to that previously. ${ }^{4}{ }^{1} \mathbf{H}$ NMR (500 $\left.\mathrm{MHz}, \mathrm{CDCl}_{3}\right) \delta 7.64(\mathrm{dd}, J=7.8,1.3 \mathrm{~Hz}, 1 \mathrm{H}), 7.35-7.30(\mathrm{~m}, 2 \mathrm{H}), 7.30-7.20(\mathrm{~m}, 6 \mathrm{H}), 7.12(\mathrm{dd}, J=$ $7.5,0.9 \mathrm{~Hz}, 1 \mathrm{H}), 3.19(\mathrm{~d}, J=13.8 \mathrm{~Hz}, 1 \mathrm{H}), 3.07(\mathrm{~d}, J=13.7 \mathrm{~Hz}, 1 \mathrm{H}), 2.90-2.75(\mathrm{~m}, 2 \mathrm{H}), 2.00-1.93$ $(\mathrm{m}, 1 \mathrm{H}), 1.93-1.85(\mathrm{~m}, 3 \mathrm{H}), 1.70-1.61(\mathrm{~m}, 1 \mathrm{H})$.

1-allyl-1,2,3,4-tetrahydronaphthalen-1-ol (S-23). The general procedure was followed to give the product as a colorless oil $(6.81 \mathrm{~g}, 90 \%)$. Spectral data is identical to that previously. ${ }^{5}{ }^{1} \mathbf{H}$ NMR (500 $\left.\mathrm{MHz} \mathrm{CDCl}_{3}\right) \delta 7.56(\mathrm{dd}, J=7.8,1.3 \mathrm{~Hz}, 1 \mathrm{H}), 7.25-7.20(\mathrm{~m}, 1 \mathrm{H}), 7.18(\mathrm{td}, J=7.4,1.5 \mathrm{~Hz}, 1 \mathrm{H}), 5.87$ $-5.77(\mathrm{~m}, 1 \mathrm{H}), 5.17-5.14(\mathrm{~m}, 1 \mathrm{H}), 5.14-5.11(\mathrm{~m}, 1 \mathrm{H}), 2.87-2.71(\mathrm{~m}, 2 \mathrm{H}), 2.61(\mathrm{dt}, J=7.3,1.1 \mathrm{~Hz}$, $2 \mathrm{H}), 2.10-2.01(\mathrm{~m}, 1 \mathrm{H}), 1.96(\mathrm{~s}, 1 \mathrm{H}), 1.93-1.77(\mathrm{~m}, 3 \mathrm{H})$.

1-ethynyl-1,2,3,4-tetrahydronaphthalen-1-ol (S-24). The general procedure was followed to give the product as a white solid (637 mg, 45\%). Spectral data is identical to that previously. ${ }^{6} \mathbf{H}$ NMR (500 $\left.\mathrm{MHz}, \mathrm{CDCl}_{3}\right) \delta 7.78-7.75(\mathrm{~m}, 1 \mathrm{H}), 7.28-7.21(\mathrm{~m}, 3 \mathrm{H}), 7.12-7.09(\mathrm{~m}, 1 \mathrm{H}), 2.89-2.75(\mathrm{~m}, 2 \mathrm{H}), 2.60$ (s, 1H), $2.27(\mathrm{~s}, 1 \mathrm{H}), 2.22(\mathrm{dd}, J=7.3,4.7 \mathrm{~Hz}, 2 \mathrm{H}), 2.08-1.98(\mathrm{~m}, 1 \mathrm{H}), 1.98-1.89(\mathrm{~m}, 1 \mathrm{H})$.

1-phenyl-1,2,3,4-tetrahydronaphthalen-1-ol (S-25). The general procedure was followed to give the product as a colorless oil $(3.59 \mathrm{~g}, 51 \%)$. Spectral data is identical to that previously. ${ }^{7}{ }^{1} \mathbf{H}$ NMR (500 $\left.\mathrm{MHz}, \mathrm{CDCl}_{3}\right) \delta 7.36-7.28(\mathrm{~m}, 4 \mathrm{H}), 7.27-7.16(\mathrm{~m}, 3 \mathrm{H}), 7.15-7.10(\mathrm{~m}, 1 \mathrm{H}), 7.05(\mathrm{dd}, J=7.8,1.0 \mathrm{~Hz}$, $1 \mathrm{H}), 2.96-2.85(\mathrm{~m}, 2 \mathrm{H}), 2.18(\mathrm{~d}, J=5.0 \mathrm{~Hz}, 1 \mathrm{H}), 2.18-2.09(\mathrm{~m}, 2 \mathrm{H}), 2.05-1.96(\mathrm{~m}, 1 \mathrm{H}), 1.85-1.76$ $(\mathrm{m}, 1 \mathrm{H})$. 


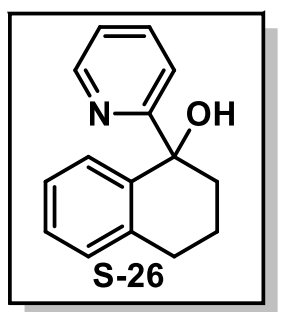

1-(pyridin-2-yl)-1,2,3,4-tetrahydronaphthalen-1-ol (S-26). 2-Bromopyridine (1.2 mL, $12.5 \mathrm{mmol}$ ) was added to a flame-dried flask under argon and dissolved in dry THF $(20 \mathrm{~mL})$. The solution was cooled to $-78^{\circ} \mathrm{C}$ then $n$-BuLi (5.8 mL, 14.5; $2.5 \mathrm{M}$ in hexanes) was added and the solution was stirred for $2 \mathrm{~h}$. Next, $\alpha$-tetralone $(665 \mu \mathrm{L}, 5.0 \mathrm{mmol})$ in THF $(5 \mathrm{~mL})$ was added to the solution. The reaction was slowly warmed to room temperature and stirred overnight. After the reaction was complete by TLC, the solution was cooled to $0^{\circ} \mathrm{C}$ and the reaction was quenched by the addition of water $(15 \mathrm{~mL})$. The two layers were separated and the aqueous layer was extracted with EtOAc (2x $20 \mathrm{~mL})$. The combined organic layers were dried over $\mathrm{Na}_{2} \mathrm{SO}_{4}$, filtered, and concentrated. The crude oil was purified by column chromatography (9;1 $\rightarrow$ 8:2 EtOAc:hexanes) to give $\mathbf{S - 2 6}$ as a yellow solid (987 mg, 88\%). Mp: $72-76^{\circ} \mathrm{C}$; IR (film): 3345, 2932, 2860, 1591, 1086, $769 \mathrm{~cm}^{-1}$; $\mathbf{R}_{\mathrm{f}} 0.34$ (3:7 EtOAc-hexanes); ${ }^{1} \mathrm{H}$ NMR $\left(500 \mathrm{MHz}, \mathrm{CDCl}_{3}\right) \delta 8.58(\mathrm{ddd}, J=4.9,1.7,1.0 \mathrm{~Hz}, 1 \mathrm{H}), 7.61-7.57$ (td, $\left.J=5.0,2.0 \mathrm{~Hz}\right), 7.23-7.19(\mathrm{~m}, 1 \mathrm{H}), 7.18$ - $7.14(\mathrm{~m}, J=6.8,3.6 \mathrm{~Hz}, 2 \mathrm{H}), 7.10-7.05(\mathrm{~m}, 1 \mathrm{H}), 6.98(\mathrm{dt}, J=8.0,1.0 \mathrm{~Hz}, 1 \mathrm{H}), 6.91-6.87(\mathrm{~m}, 1 \mathrm{H}), 5.85(\mathrm{~s}, 1 \mathrm{H}), 3.02-$ $2.84(\mathrm{~m}, 2 \mathrm{H}), 2.19-2.01(\mathrm{~m}, 3 \mathrm{H}), 1.92-1.82(\mathrm{~m}, 1 \mathrm{H}) ;{ }^{13} \mathrm{C}$ NMR $\left(125 \mathrm{MHz}, \mathrm{CDCl}_{3}\right) \delta$ 165.7, 146.9, 140.4, 137.8, 136.4, 129.1, 128.7, 127.2, 126.2, 121.9, 121.5, 73.9, 40.5, 29.7, 19.6; HRMS (ESI) $\mathrm{m} / \mathrm{z}$ calcd. for $\mathrm{C}_{15} \mathrm{H}_{14} \mathrm{~N}^{+}(\mathrm{M}-\mathrm{OH})^{+} 208.1126$; found: 208.1129 .

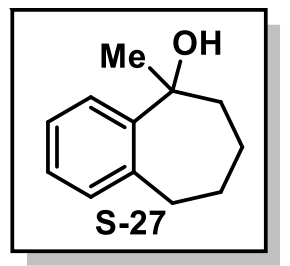

5-methyl-6,7,8,9-tetrahydro-5H-benzo[b]annulen-5-ol (S-27). The general procedure was followed to give S-27 as a white solid (859 mg, 75\%). Mp: $47-51^{\circ} \mathrm{C}$; IR (neat): 3388, 3334, 2929, 2855, 1439, 1102, $756 \mathrm{~cm}^{-1} ; \mathbf{R}_{\mathbf{f}} 0.33$ (3:7 EtOAc-hexanes); ${ }^{1} \mathbf{H} \mathbf{N M R}\left(500 \mathrm{MHz}, \mathrm{CDCl}_{3}\right) \delta 7.69$ (dd, $J=7.8,1.0 \mathrm{~Hz}$, $1 \mathrm{H}), 7.22(\mathrm{td}, J=7.6,1.4 \mathrm{~Hz}, 1 \mathrm{H}), 7.15(\mathrm{td}, J=7.3,1.4 \mathrm{~Hz}, 1 \mathrm{H}), 7.09(\mathrm{~d}, J=7.3 \mathrm{~Hz}, 1 \mathrm{H}), 3.02-2.85$ (m, 2H), $2.03-1.74(\mathrm{~m}, 6 \mathrm{H}), 1.61(\mathrm{~s}, 4 \mathrm{H}) ;{ }^{13} \mathrm{C}$ NMR $\left(125 \mathrm{MHz}, \mathrm{CDCl}_{3}\right) \delta 147.1,140.0,130.8,126.9$, 126.1, 125.1, 75.6, 42.5, 36.8, 28.7, 27.9, 26.1; HRMS (ESI) $\mathrm{m} / z$ calcd. for $\mathrm{C}_{12} \mathrm{H}_{15}{ }^{+}(\mathrm{M}-\mathrm{OH})^{+}$159.1174; found: 159.1174.

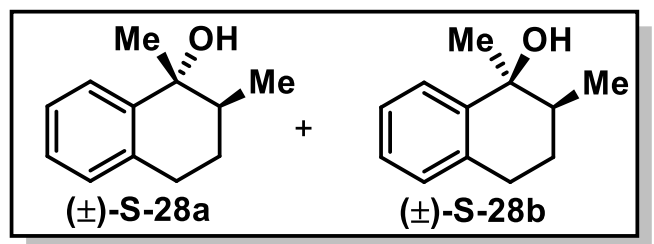

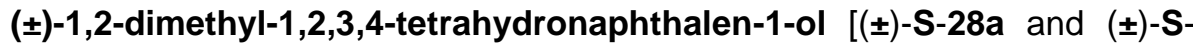
28b). The general procedure was followed to give ( $\mathbf{\pm})$-S-28a and ( $\mathbf{\mathbf { t }})-\mathbf{S}-\mathbf{2 8 b}$ (360 mg, 55\%). Mp $59-61^{\circ} \mathrm{C}$; IR (neat): 3303, 2972, 2923, 1487, 1457, 1433, 917, 760, $729 \mathrm{~cm}^{-1} ; \mathbf{R}_{\mathbf{f}} 0.30$ (3:7 EtOAc-hexanes); ${ }^{1} \mathbf{H} \mathbf{~ N M R}\left(500 \mathrm{MHz}, \mathrm{CDCl}_{3}\right)$ $\delta 7.67-7.62(\mathrm{~m}, 2 \mathrm{H}), 7.25-7.20(\mathrm{~m}, 2 \mathrm{H}), 7.17(\mathrm{qd}, J=7.5,1.5 \mathrm{~Hz}, 2 \mathrm{H}), 7.10$ $-7.04(\mathrm{~m}, 2 \mathrm{H}), 2.93-2.81(\mathrm{~m}, 3 \mathrm{H}), 2.81-2.72(\mathrm{~m}, 1 \mathrm{H}), 1.97-1.83(\mathrm{~m}, 4 \mathrm{H}), 1.75-1.59(\mathrm{~m}, 4 \mathrm{H}), 1.58(\mathrm{~s}, 3 \mathrm{H}), 1.37(\mathrm{~s}$, $3 \mathrm{H}), 1.11(\mathrm{~d}, J=4.6 \mathrm{~Hz}, 3 \mathrm{H}), 1.10(\mathrm{~d}, J=4.7 \mathrm{~Hz}, 3 \mathrm{H}) ;{ }^{13} \mathrm{C}$ NMR $\left(125 \mathrm{MHz}, \mathrm{CDCl}_{3}\right) \delta$ 144.6, 142.4, 135.9, 135.6, 128.6, 128.4, 126.9, 126.8, 126.7, 126.3, 126.2, 126.1, 73.9, 72.2, 41.0, 39.3, 29.3, 28.8, 28.5, 27.7, 27.2, 24.8, 15.4, 14.7; HRMS (ESI) $\mathrm{m} / z$ calcd. for $\mathrm{C}_{12} \mathrm{H}_{15^{+}}(\mathrm{M}-\mathrm{OH})^{+}$159.1174; found: 159.1186 .

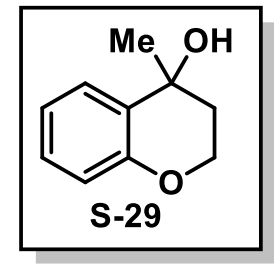

4-methylchroman-4-ol (S-29). The general procedure was followed to give S-29 (779 mg, 84\%) was a white solid. $\mathbf{m p}$ : $93-95^{\circ} \mathrm{C}$; IR (neat): 3294, 2982, 1488, 1223, 1134, 1053, $754 \mathrm{~cm}^{-1} ; \mathbf{R}_{\mathbf{f}} 0.34$ (3:7 EtOAc-hexanes); ${ }^{1} \mathbf{H}$ NMR $\left(500 \mathrm{MHz}, \mathrm{CDCl}_{3}\right) \delta 7.48$ (dd, $\left.J=7.8,1.7 \mathrm{~Hz}, 1 \mathrm{H}\right), 7.17$ (ddd, $J=8.3,7.2$, $1.7 \mathrm{~Hz}, 1 \mathrm{H}), 6.95-6.91(\mathrm{~m}, 1 \mathrm{H}), 6.82(\mathrm{dd}, J=8.2,1.2 \mathrm{~Hz}, 1 \mathrm{H}), 4.28$ (ddd, $J=11.3,8.0,4.3 \mathrm{~Hz}, 1 \mathrm{H}$ ), 4.22 (ddd, $J=11.2,5.6,4.2 \mathrm{~Hz}, 1 \mathrm{H}), 2.09-2.05(\mathrm{~m}, 2 \mathrm{H}), 1.97(\mathrm{~s}, 1 \mathrm{H}), 1.62(\mathrm{~s}, 3 \mathrm{H}) ;{ }^{13} \mathrm{C}$ NMR (125 $\left.\mathrm{MHz}, \mathrm{CDCl}_{3}\right) \delta 153.8,129.1,128.4,126.3,120.7,117.0,66.3,63.2,38.0,29.4 ;$ HRMS (ESI) $\mathrm{m} / z$ calcd. for $\mathrm{C}_{10} \mathrm{H}_{11} \mathrm{O}^{+}(\mathrm{M}-\mathrm{OH})^{+}$147.0810; found: 147.0821 . 


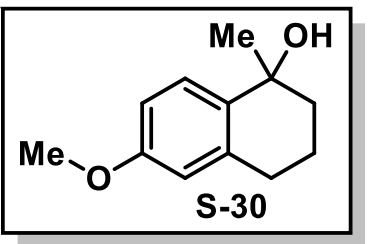

6-methoxy-1-methyl-1,2,3,4-tetrahydronaphthalen-1-ol (S-30). The general procedure was followed to give S-30 (712 mg, 74\%) as a white amorphous solid. IR (film): 3503, 2981, 2937, 1499, 1372, 1044, $844 \mathrm{~cm}^{-1} ; \mathbf{R}_{\mathbf{f}} 0.21$ (3:7 EtOAc-hexanes); ${ }^{1} \mathbf{H}$ NMR $\left(500 \mathrm{MHz}, \mathrm{CDCl}_{3}\right) \delta 7.49$ (d, $J=8.7 \mathrm{~Hz}, 1 \mathrm{H}), 6.76(\mathrm{dd}, J=8.7,2.8 \mathrm{~Hz}, 1 \mathrm{H}), 6.57(\mathrm{~d}, J=2.7 \mathrm{~Hz}, 1 \mathrm{H}), 3.77(\mathrm{~s}, 3 \mathrm{H}), 2.82-$ $2.67(\mathrm{~m}, 2 \mathrm{H}), 1.96-1.85(\mathrm{~m}, 4 \mathrm{H}), 1.83-1.75(\mathrm{~m}, 1 \mathrm{H}), 1.53(\mathrm{~s}, 3 \mathrm{H}) ;{ }^{13} \mathbf{C ~ N M R}\left(125 \mathrm{MHz}, \mathrm{CDCl}_{3}\right)$ ठ 158.3, 137.7, 135.2, 127.6, 112.9, 112.6, 70.1, 55.1, 39.8, 30.6, 30.3, 20.3; HRMS (ESI) $\mathrm{m} / z$ calcd. for $\mathrm{C}_{12} \mathrm{H}_{15} \mathrm{O}^{+}(\mathrm{M}-\mathrm{OH})^{+}$ 175.1123; found: 175.1154 .

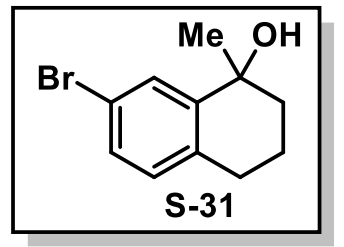

7-bromo-1-methyl-1,2,3,4-tetrahydronaphthalen-1-ol (S-31). The general procedure was followed to give S-31 (482 $\mathrm{mg}, 55 \%$ ) as a tan solid. Mp: $88-93^{\circ} \mathrm{C}$; IR (neat): $3326,2972,2935$, 2865, 1474, 1103, 929, 856, 731, $626 \mathrm{~cm}^{-1} ; \mathbf{R}_{\mathbf{f}} 0.45$ (7:3 hexanes-EtOAc); ${ }^{1} \mathbf{H}$ NMR $(500 \mathrm{MHz}$, $\left.\mathrm{CDCl}_{3}\right) \delta 7.70(\mathrm{~d}, J=2.0 \mathrm{~Hz}, 1 \mathrm{H}), 7.25(\mathrm{dd}, J=8.2,2.1 \mathrm{~Hz}, 1 \mathrm{H}), 6.93(\mathrm{~d}, J=8.2 \mathrm{~Hz}, 1 \mathrm{H}), 2.78-$ $2.64(\mathrm{~m}, 2 \mathrm{H}), 2.08-1.73(\mathrm{~m}, 5 \mathrm{H}), 1.51(\mathrm{~s}, 3 \mathrm{H}) ;{ }^{13} \mathrm{C} \mathrm{NMR}\left(125 \mathrm{MHz}, \mathrm{CDCl}_{3}\right) \delta 145.0,135.0,130.4$, 130.0, 129.3, 119.7, 70.4, 39.3, 30.7, 29.2, 20.1; HRMS (ESI) m/z calcd. for $\mathrm{C}_{11} \mathrm{H}_{12} \mathrm{BrO}^{-}(\mathrm{M}-\mathrm{H})^{-}{ }^{-} 239.0072$; found: 239.0092.

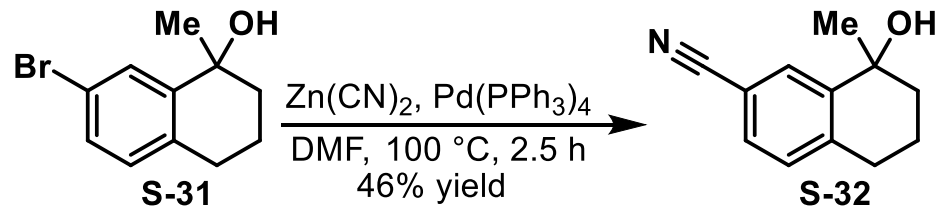

\section{8-hydroxy-8-methyl-5,6,7,8-tetrahydronaphthalene-2-carbonitrile}

(S-32).

7-bromo-1-methyl-1,2,3,4tetrahydronaphthalen-1-ol $\quad(\mathbf{S}-31) \quad(429 \mathrm{mg}, \quad 1.78 \mathrm{mmol}), \quad$ zinc(II) $\quad$ cyanide $(219 \mathrm{mg}, \quad 1.86 \quad \mathrm{mmol}), \quad$ and tetrakis(triphenylphosphine)palladium(0) $(195 \mathrm{mg}, 0.169 \mathrm{mmol}$ ) were added to a flame-dried flask under argon and dissolved in dry DMF (3 mL). The solution was heated to $100^{\circ} \mathrm{C}$ for $2.5 \mathrm{~h}$. After the reaction was complete by TLC, the reaction was diluted with 1:1 $\mathrm{MeCN}: \mathrm{MeOH}(60 \mathrm{~mL})$. The solution was filtered over a pad of celite and rinsed with 1:1 $\mathrm{MeCN}: \mathrm{MeOH}(2 \times 25 \mathrm{~mL})$. The filtrated was concentrated. The crude oil was purified by column chromatography (3:7 EtOAc:hexanes) to give the desired product as a yellow solid (154 mg, 46\%). Mp: $90-94{ }^{\circ} \mathrm{C}$; IR (neat): 3295, 2952, 2927, 2224, 1492, 993, 907, 861, 819, $735 \mathrm{~cm}^{-1} ; \mathbf{R}_{\mathbf{f}} 0.30$ (7:3 hexanes-EtOAc); ${ }^{1} \mathbf{H}$ NMR (500 MHz, CDCl $) \delta 7.91(\mathrm{~d}, J=1.1 \mathrm{~Hz}$, $1 \mathrm{H}), 7.41(\mathrm{dd}, J=7.9,1.6 \mathrm{~Hz}, 1 \mathrm{H}), 7.16(\mathrm{~d}, J=7.9 \mathrm{~Hz}, 1 \mathrm{H}), 2.90-2.77(\mathrm{~m}, 2 \mathrm{H}), 2.03-1.75(\mathrm{~m}, 5 \mathrm{H}), 1.54(\mathrm{~s}, 3 \mathrm{H}) ;{ }^{13} \mathrm{C} \mathrm{NMR}$ $\left(125 \mathrm{MHz}, \mathrm{CDCl}_{3}\right) \delta 144.2,141.7,130.7,130.0,129.6,119.0,110.0,77.2,76.9,76.6,70.2,39.1,30.8,29.8,19.9$; HRMS (ESI) $\mathrm{m} / z$ calcd. for $\mathrm{C}_{12} \mathrm{H}_{14} \mathrm{NO}^{+}(\mathrm{M}+\mathrm{H})^{+}$188.1075; found: 188.1094 .

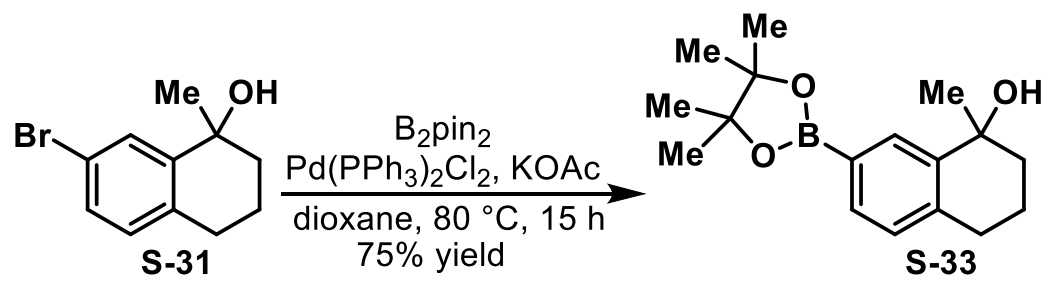

1-methyl-7-(4,4,5,5-tetramethyl-1,3,2-dioxaborolan-2-yl)-1,2,3,4-tetrahydronaphthalen-1-ol (S-33). 7-bromo-1-methyl1,2,3,4-tetrahydronaphthalen-1-ol (S-31) (788 mg, $3.27 \mathrm{mmol}$ ), bis(pinacolato)diboron (993 mg, $3.91 \mathrm{mmol}$ ), bis(triphenylphosphine)palladium(II) dichloride $(70.5 \mathrm{mg}, 0.100 \mathrm{mmol})$, and potassium acetate (966 $\mathrm{mg}, 9.84 \mathrm{mmol}$ ) were added to a flame-dried flask under argon and dissolved in dioxane $(35 \mathrm{~mL})$. The solution was heated to $80^{\circ} \mathrm{C}$ for $15 \mathrm{~h}$. After the reaction was complete by TLC, the reaction was concentrated. The black residue was purified by column 
chromatography $(2: 8 \rightarrow 3: 7$ EtOAc:hexanes) to give the desired product as a colorless foam (708 mg, $75 \%)$. Mp: $39-46$ ${ }^{\circ} \mathrm{C}$; IR (neat): 3420, 2979, 2932, 1457, 1359, 1142, 1092, 1067, 963, 852, $686 \mathrm{~cm}^{-1} ; \mathbf{R}_{\mathbf{f}} 0.38$ (7:3 hexanes-EtOAc); ${ }^{1} \mathbf{H}$ NMR $\left(500 \mathrm{MHz}, \mathrm{CDCl}_{3}\right) \delta 8.05(\mathrm{~s}, 1 \mathrm{H}), 7.60(\mathrm{~d}, J=7.5 \mathrm{~Hz}, 1 \mathrm{H}), 7.08(\mathrm{~d}, J=7.6 \mathrm{~Hz}, 1 \mathrm{H}), 2.89-2.72(\mathrm{~m}, 2 \mathrm{H}), 1.99-1.87(\mathrm{~m}$, 4H), $1.86-1.74(\mathrm{~m}, 1 \mathrm{H}), 1.59(\mathrm{~s}, 3 \mathrm{H}), 1.35-1.32(\mathrm{~m}, 13 \mathrm{H}) ;{ }^{13} \mathrm{C} \mathrm{NMR}\left(125 \mathrm{MHz}, \mathrm{CDCl}_{3}\right) \delta$ 142.1, 139.6, 133.2, 132.7, 128.2, 83.5, 70.4, 39.6, 30.7, 30.1, 24.9, 24.8, 24.7, 20.1; HRMS (ESI) $\mathrm{m} / \mathrm{z}$ calcd. for $\mathrm{C}_{17} \mathrm{H}_{25} \mathrm{BNaO}_{3}{ }^{+}(\mathrm{M}+\mathrm{Na})^{+} 311.1794$; found: 311.1820 .

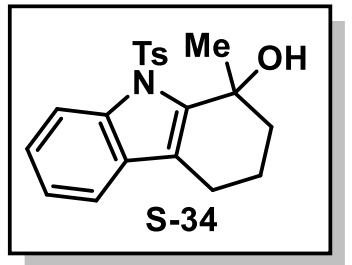

1-methyl-9-tosyl-2,3,4,9-tetrahydro-1H-carbazol-1-ol (S-34). The general procedure was followed to give S-34 as a yellow solid (1.59 g, 71\%). Mp: $117-126{ }^{\circ} \mathrm{C}$; IR (neat): 3510, 3437, 2981, 2941, 2360, 2341, 1596, 1454, 1343, 1160, 1110, 1094, 934, 752, $666 \mathrm{~cm}^{-1} ; \mathbf{R}_{\mathbf{f}} 0.55(7: 3$ hexanes-EtOAc); ${ }^{1} \mathbf{H}$ NMR $\left(500 \mathrm{MHz}, \mathrm{CDCl}_{3}\right) \delta 7.98(\mathrm{~d}, J=8.3 \mathrm{~Hz}, 1 \mathrm{H}), 7.69-7.65(\mathrm{~m}, 2 \mathrm{H}), 7.33$ - $7.29(\mathrm{~m}, 1 \mathrm{H}), 7.24(\mathrm{dd}, J=8.3,1.3 \mathrm{~Hz}, 1 \mathrm{H}), 7.19(\mathrm{dd}, J=7.5,0.9 \mathrm{~Hz}, 1 \mathrm{H}), 7.10(\mathrm{~d}, J=8.1 \mathrm{~Hz}$, 2H), $4.78(\mathrm{~s}, 1 \mathrm{H}), 2.66(\mathrm{dt}, J=16.7,5.7 \mathrm{~Hz}, 1 \mathrm{H}), 2.58$ (ddd, $J=16.6,7.2,5.4 \mathrm{~Hz}, 1 \mathrm{H}), 2.28(\mathrm{~s}, 3 \mathrm{H}$ ), 2.22 (ddd, $J=13.0,8.7,2.6 \mathrm{~Hz}, 1 \mathrm{H}), 2.11-2.02(\mathrm{~m}, 1 \mathrm{H}), 1.90-1.85(\mathrm{~m}, 1 \mathrm{H}), 1.83(\mathrm{~s}, 3 \mathrm{H}) ;{ }^{13} \mathrm{C} \mathrm{NMR}\left(125 \mathrm{MHz}, \mathrm{CDCl}_{3}\right) \delta$ 144.4, 140.8, 137.4, 134.2, 130.2, 129.3, 126.6, 125.2, 123.9, 119.0, 115.7, 77.2, 76.9, 76.6, 68.7, 41.2, 30.0, 22.4, 21.4, 19.6; HRMS (ESI) $m / z$ calcd. for $\mathrm{C}_{20} \mathrm{H}_{21} \mathrm{NNaO}_{3} \mathrm{~S}^{+}(\mathrm{M}+\mathrm{Na})^{+} 378.1140$; found: 378.1177 .

\section{General Procedure for oxidative rearrangement:}

To a flame-dried vial containing activated $3 \AA$ molecular sieves, under argon, was added the $3^{\circ}-\mathrm{OH}(1$ equiv) dissolved in dry $\mathrm{CH}_{2} \mathrm{Cl}_{2}(0.2 \mathrm{M})$ and the solution was cooled to $-25^{\circ} \mathrm{C}$. [ $\left.\left(\mathrm{Py}^{+}\right)_{2} \mathrm{IPh}\right] 2 \mathrm{OTf}$ (1.5 equiv), dissolved in dry HFIP (20 equiv), was slowly added to the suspension and the reaction was monitored by TLC. Upon completion, the reaction was filtered and the filtrate was collected in a round bottom flask that was cooled to $0{ }^{\circ} \mathrm{C}$. The cooled filtrate was then concentrated by rotatory evaporation while maintaining the $0{ }^{\circ} \mathrm{C}$ temperature of the flask. Next, the solution was purified by flash chromatography to give the desired acetal or enol ether.

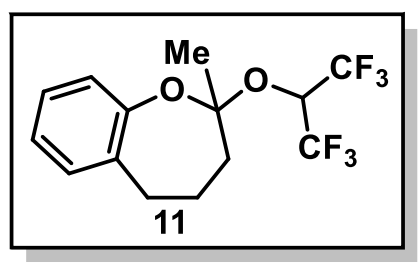

2-((1,1,1,3,3,3-hexafluoropropan-2-yl)oxy)-2-methyl-2,3,4,5-tetrahydrobenzo[b]oxepine (11). Purified by FCC in 1:19 EtOAc:Hexanes to give 11 (100 mg, 84\%) as a colorless oil. IR (film) 2947, 2323, 1489, 1357, 1099, 764, $687 \mathrm{~cm}^{-1} ; \mathbf{R}_{\mathbf{f}} 0.85$ (3:7 EtOAc-hexanes); ${ }^{1} \mathbf{H}$ NMR $\left(500 \mathrm{MHz}, \mathrm{CDCl}_{3}\right) \delta 7.16(\mathrm{td}, J=7.6,1.7 \mathrm{~Hz}, 1 \mathrm{H}), 7.12-7.09(\mathrm{~m}, 1 \mathrm{H}), 7.05(\mathrm{td}, J=7.4,1.1$ $\mathrm{Hz}, 1 \mathrm{H}), 6.95(\mathrm{~d}, J=7.9 \mathrm{~Hz}, 1 \mathrm{H}), 5.01$ (sept, $J=6.1 \mathrm{~Hz}, 1 \mathrm{H}), 2.84(\mathrm{~m}, 1 \mathrm{H}), 2.71(\mathrm{~m}, 1 \mathrm{H}), 2.01$ $(\mathrm{m}, 2 \mathrm{H}), 1.98-1.91(\mathrm{~m}, 1 \mathrm{H}), 1.62(\mathrm{~m}, 1 \mathrm{H}), 1.36(\mathrm{~s}, 3 \mathrm{H}) ;{ }^{13} \mathrm{C}$ NMR $\left(125 \mathrm{MHz}, \mathrm{CDCl}_{3}\right) \delta 152.9,134.5,129.9,127.5,124.7$, 122.2, 105.7, 69.2, 68.9, 68.6, 68.4, 68.1, 38.7, 31.3, 22.8, 21.6; HRMS (ESI) $\mathrm{m} / \mathrm{z}$ calcd. for $\mathrm{C}_{11} \mathrm{H}_{13} \mathrm{O}^{+}\left(\mathrm{M}-\mathrm{OCH}\left(\mathrm{CF}_{3}\right)_{2}\right)^{+}$ 161.0966; found: 161.0967

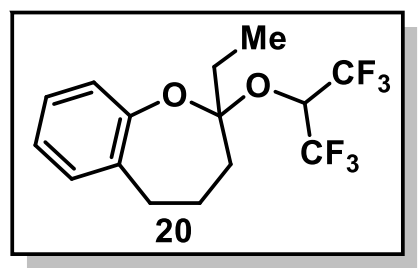

2-ethyl-2-((1,1,1,3,3,3-hexafluoropropan-2-yl)oxy)-2,3,4,5-tetrahydrobenzo[b]oxepine (20). Purified by FCC in 1:19 EtOAc:Hexanes to give $20(81 \mathrm{mg}, 66 \%)$ as a colorless oil. IR (film) 2981, 2936, 2360, 2324, 1456, 1373, 1100, 754, $686 \mathrm{~cm}^{-1} ; \mathbf{R}_{\mathbf{f}} 0.71$ (3:7 EtOAc-hexanes); ${ }^{1} \mathrm{H}$ NMR $\left(500 \mathrm{MHz}, \mathrm{CDCl}_{3}\right) \delta 7.16(\mathrm{td}, J=7.5,1.6 \mathrm{~Hz}, 1 \mathrm{H}), 7.10(\mathrm{dd}, J=7.4,1.6 \mathrm{~Hz}, 1 \mathrm{H}), 7.04$ (td, $J=7.4,1.2 \mathrm{~Hz}, 1 \mathrm{H}), 6.95(\mathrm{dd}, J=7.9,1.0 \mathrm{~Hz}, 1 \mathrm{H}), 5.04$ (sept, $J=6.1 \mathrm{~Hz}, 1 \mathrm{H}), 2.82(\mathrm{~m}$, $1 \mathrm{H}), 2.73(\mathrm{~m}, 1 \mathrm{H}), 2.14-2.06(\mathrm{~m}, 1 \mathrm{H}), 1.96-1.84(\mathrm{~m}, 2 \mathrm{H}), 1.69-1.62(\mathrm{~m}, 2 \mathrm{H}), 1.62-1.51(\mathrm{~m}, 2 \mathrm{H}), 0.93(\mathrm{t}, J=7.4 \mathrm{~Hz}$, 3H); ${ }^{13} \mathrm{C}$ NMR $\left(125 \mathrm{MHz}, \mathrm{CDCl}_{3}\right) \delta 152.9,134.1,130.0,127.5,124.5,122.3,107.3,35.5,31.9,28.9,21.2,7.5$; HRMS (ESI) $\mathrm{m} / \mathrm{z}$ calcd. for $\mathrm{C}_{12} \mathrm{H}_{15} \mathrm{O}^{+}\left(\mathrm{M}-\mathrm{OCH}\left(\mathrm{CF}_{3}\right)_{2}\right)^{+} 175.1123$; found: 175.1141 
2-((1,1,1,3,3,3-hexafluoropropan-2-yl)oxy)-2-isopropyl-2,3,4,5-

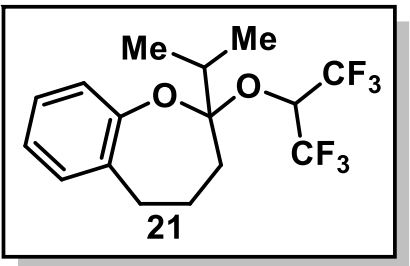

tetrahydrobenzo[b]oxepine (21). Purified by FCC in 1:19 EtOAc:Hexanes to give 21 (67 mg, $54 \%$ ) as a colorless oil. IR (film) 2971, 2941, 2323, 1488, 1456, 1098, 764, $686 \mathrm{~cm}^{-1} ; \mathbf{R}_{\mathbf{f}} 0.47$ (3:7 EtOAc-hexanes); ${ }^{1} \mathbf{H}$ NMR $\left(500 \mathrm{MHz}, \mathrm{CDCl}_{3}\right) \delta 7.18$ (td, $\left.J=7.7,1.8 \mathrm{~Hz}, 1 \mathrm{H}\right), 7.09$ (dd, $J$ $=7.7,1.8 \mathrm{~Hz}, 1 \mathrm{H}), 7.05(\mathrm{~m}, 2 \mathrm{H}), 4.87$ (sept, $J=6.0 \mathrm{~Hz}, 1 \mathrm{H}), 2.85-2.78(\mathrm{~m}, 1 \mathrm{H}), 2.72-2.65$ $(\mathrm{m}, 1 \mathrm{H}), 2.18-2.11(\mathrm{~m}, 1 \mathrm{H}), 2.09-2.02(\mathrm{~m}, 1 \mathrm{H}), 1.89-1.76(\mathrm{~m}, 2 \mathrm{H}), 1.74-1.63(\mathrm{~m}, 1 \mathrm{H})$, $1.05(\mathrm{~d}, J=6.6 \mathrm{~Hz}, 3 \mathrm{H}), 0.98(\mathrm{~d}, J=6.9 \mathrm{~Hz}, 3 \mathrm{H}) ;{ }^{13} \mathrm{C}$ NMR $\left(125 \mathrm{MHz}, \mathrm{CDCl}_{3}\right) \delta$ 153.4, 133.3, 130.0, 127.7, 124.1, 122.2, 108.3, 34.0, 32.7, 32.1, 20.9, 17.4, 14.7; HRMS (ESI) $\mathrm{m} / z$ calcd. for $\mathrm{C}_{13} \mathrm{H}_{17} \mathrm{O}^{+}\left(\mathrm{M}-\mathrm{OCH}\left(\mathrm{CF}_{3}\right)_{2}\right)^{+}$189.1279; found: 187.1295

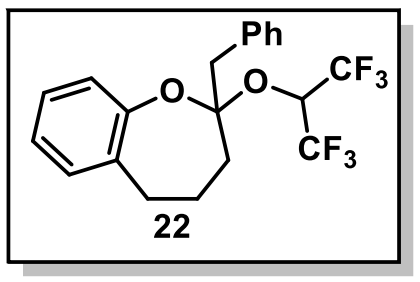

2-benzyl-2-((1,1,1,3,3,3-hexafluoropropan-2-yl)oxy)-2,3,4,5-tetrahydrobenzo[b]oxepine (22). Purified by FCC in 1:9 EtOAc:Hexanes to give 22 (115 mg, 78\%) as white solid. Mp: $72-$ $75^{\circ} \mathrm{C}$; IR (neat) 3031, 2930, 2851, 2323, 1352, 1098, 765, $685 \mathrm{~cm}^{-1} ; \mathbf{R}_{\mathfrak{f}} 0.69(2: 8 \mathrm{EtOAc-}$ hexanes); ${ }^{1} \mathrm{H}$ NMR $\left(500 \mathrm{MHz}, \mathrm{CDCl}_{3}\right) \delta 7.28-7.21(\mathrm{~m}, 5 \mathrm{H}), 7.18(\mathrm{td}, J=7.6,1.7 \mathrm{~Hz}, 1 \mathrm{H})$, 7.14 (dd, $J=7.4,1.6 \mathrm{~Hz}, 1 \mathrm{H}), 7.07$ (td, $J=7.4,1.2 \mathrm{~Hz}, 1 \mathrm{H}), 7.00$ (dd, $J=7.9,1.0 \mathrm{~Hz}, 1 \mathrm{H}$ ), 7.00 (dd, $J=7.9,1.0 \mathrm{~Hz}, 1 \mathrm{H}$ ), 5.13 (sept, $J=6.1 \mathrm{~Hz}, 1 \mathrm{H}$ ), 3.02 (dd, $J=14.0,1.1 \mathrm{~Hz}, 1 \mathrm{H}), 2.90(\mathrm{~d}, J=14.0 \mathrm{~Hz}, 1 \mathrm{H}), 2.88-$ $2.83(\mathrm{~m}, 1 \mathrm{H}$ ), 2.77 (ddd, $J=14.3,6.7,4.1 \mathrm{~Hz}, 1 \mathrm{H}$ ), 1.90 (ddt, $J=6.6,4.6,3.3 \mathrm{~Hz}, 2 \mathrm{H}$ ), 1.84 (dd, $J=13.1,7.1 \mathrm{~Hz}, 1 \mathrm{H}$ ), 1.81 $-1.69(\mathrm{~m}, 1 \mathrm{H}) ;{ }^{13} \mathrm{C}$ NMR $\left(126 \mathrm{MHz}, \mathrm{CDCl}_{3}\right) \delta 152.9,134.9,134.0,130.5,129.9,127.9,127.6,126.7,124.6,122.4,106.1$, 43.1, 35.3, 31.6, 21.6; HRMS (ESI) $\mathrm{m} / z$ calcd. for $\mathrm{C}_{17} \mathrm{H}_{17} \mathrm{O}^{+}\left(\mathrm{M}-\mathrm{OCH}\left(\mathrm{CF}_{3}\right)_{2}\right)^{+} 237.1279$; found: 237.1290

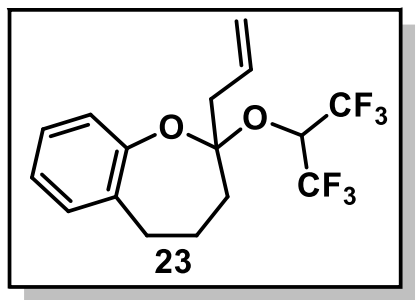

2-allyl-2-((1,1,1,3,3,3-hexafluoropropan-2-yl)oxy)-2,3,4,5-tetrahydrobenzo[b]oxepine (23). Purified by FCC in 1:19 EtOAc:Hexanes to give $\mathbf{2 3}$ (93 $\mathrm{mg}, \mathbf{7 6} \%)$ as a colorless oil. IR (film) 2957, 2323, 1644, 1489, 1353, 1098, 765, 732, $686 \mathrm{~cm}^{-1} ; \mathbf{R}_{\mathbf{f}} 0.43$ (3:7 EtOAc-hexanes); ${ }^{1} \mathrm{H}$ NMR $\left(500 \mathrm{MHz}, \mathrm{CDCl}_{3}\right) \delta 7.17$ (td, $\left.J=7.4,1.5 \mathrm{~Hz}, 1 \mathrm{H}\right), 7.12(\mathrm{dd}, J=7.4,1.7 \mathrm{~Hz}, 1 \mathrm{H}), 7.06$ (td, $J=7.4,1.2 \mathrm{~Hz}, 1 \mathrm{H}), 6.96(\mathrm{dd}, J=7.9,1.1 \mathrm{~Hz}, 1 \mathrm{H}), 5.88-5.77(\mathrm{~m}, 1 \mathrm{H}), 5.14-5.00(\mathrm{~m}$, $3 \mathrm{H}$ ), $2.88-2.80(\mathrm{~m}, 1 \mathrm{H}), 2.72(\mathrm{ddd}, J=14.3,6.3,4.6 \mathrm{~Hz}, 1 \mathrm{H}), 2.49-2.41(\mathrm{~m}, 1 \mathrm{H}), 2.38(\mathrm{dd}$, $J=14.6,8.5 \mathrm{~Hz}, 1 \mathrm{H}), 2.12-2.04(\mathrm{~m}, 1 \mathrm{H}), 1.94-1.82(\mathrm{~m}, 2 \mathrm{H}), 1.66-1.55(\mathrm{~m}, 1 \mathrm{H}) ;{ }^{13} \mathrm{C} \mathrm{NMR}\left(125 \mathrm{MHz}, \mathrm{CDCl}_{3}\right) \delta 152.6$, 133.9, 131.5, 129.9, 127.4, 124.6, 122.2, 118.9, 106.0, 41.0, 35.7, 31.4, 20.8; HRMS (ESI) $\mathrm{m} / z$ calcd. for $\mathrm{C}_{13} \mathrm{H}_{15} \mathrm{O}^{+}$ $\left(\mathrm{M}-\mathrm{OCH}\left(\mathrm{CF}_{3}\right)_{2}\right)^{+}$187.1123; found: 187.1136

2-ethynyl-2-((1,1,1,3,3,3-hexafluoropropan-2-yl)oxy)-2,3,4,5-tetrahydrobenzo[b]oxepine (24). Purified by FCC in 1:19

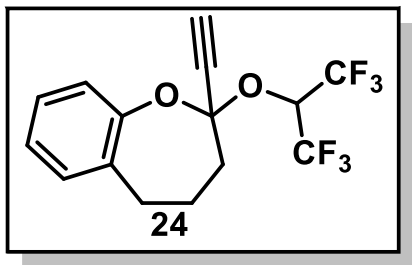
EtOAc:Hexanes to give 24 (91 mg, 75\%) as a colorless oil. IR (film) 3305, 2947, 2323, 2121, 1488, 1358, 1100, 767, 747, $687 \mathrm{~cm}^{-1} ; \mathbf{R}_{\mathbf{f}} 0.63$ (3:7 EtOAc-hexanes); ${ }^{1} \mathbf{H}$ NMR (500 MHz, $\left.\mathrm{CDCl}_{3}\right) \delta 7.19(\mathrm{td}, J=7.5,2.0 \mathrm{~Hz}, 1 \mathrm{H}), 7.15-7.06(\mathrm{~m}, 2 \mathrm{H}), 7.00(\mathrm{~d}, J=7.9 \mathrm{~Hz}, 1 \mathrm{H}), 5.14$ (sept, $J=6.0 \mathrm{~Hz}, 1 \mathrm{H}), 2.86-2.74(\mathrm{~m}, 2 \mathrm{H}), 2.67(\mathrm{~s}, 1 \mathrm{H}), 2.37-2.30(\mathrm{~m}, 1 \mathrm{H}), 2.22(\mathrm{~m}, 1 \mathrm{H})$, $2.01-1.92(\mathrm{~m}, 1 \mathrm{H}), 1.89-1.77(\mathrm{~m}, 1 \mathrm{H}) ;{ }^{13} \mathrm{C} \mathrm{NMR}\left(125 \mathrm{MHz}, \mathrm{CDCl}_{3}\right) \delta$ 152.1, 129.6, 127.3, 125.1, 122.6, 101.6, 79.3, 40.5, 31.5, 22.0; HRMS (ESI) $\mathrm{m} / \mathrm{z}$ calcd. for $\mathrm{C}_{12} \mathrm{H}_{11} \mathrm{O}^{+}\left(\mathrm{M}-\mathrm{OCH}\left(\mathrm{CF}_{3}\right)_{2}\right)^{+}$171.0810; found: 171.0822 


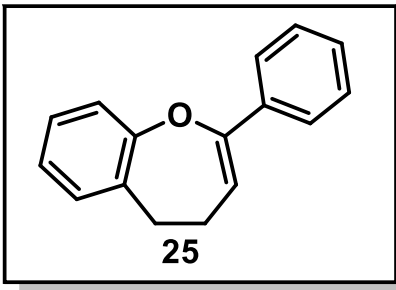

2-phenyl-4,5-dihydrobenzo[b]oxepine (25). Purified by FCC in 1:19 ether:pentane to give $25(53 \mathrm{mg}, 66 \%)$ as a colorless oil.The ${ }^{1} \mathrm{H}$ NMR and ${ }^{13} \mathrm{C}$ NMR matches that of the known compound. ${ }^{8}{ }^{1} \mathrm{H}$ NMR $\left(500 \mathrm{MHz}, \mathrm{CDCl}_{3}\right) \delta 7.72(\mathrm{~m}, 2 \mathrm{H}), 7.42-7.35(\mathrm{~m}, 2 \mathrm{H}), 7.34-7.29(\mathrm{~m}$, 1H), $7.24-7.16(\mathrm{~m}, 3 \mathrm{H}), 7.08$ (ddd, $J=7.6,6.0,2.5 \mathrm{~Hz}, 1 \mathrm{H}$ ), 5.53 (t, $J=4.6 \mathrm{~Hz}, 1 \mathrm{H}$ ), $3.15-$ $3.10(\mathrm{~m}, 2 \mathrm{H}), 2.57$ (dd, $J=11.9,4.9 \mathrm{~Hz}, 2 \mathrm{H}) ;{ }^{13} \mathrm{C} \mathrm{NMR}\left(125 \mathrm{MHz}, \mathrm{CDCl}_{3}\right) \delta$ 158.4, 152.4, $137.4,134.7,129.3,128.1,127.8,127.0,125.1,124.2,120.2,106.4,30.6,27.8$

2-(2-((1,1,1,3,3,3-hexafluoropropan-2-yl)oxy)-2,3,4,5-tetrahydrobenzo[b]oxepin-2-

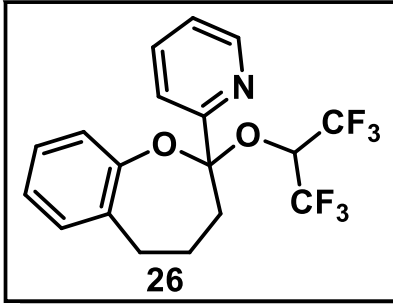

yl)pyridine (26). Purified by FCC in 2:8 EtOAc:Hexanes to give 26 (73 mg, 50\%) as an amorphous, pale-yellow solid. IR (film) 3063, 2950, 2867, 2323, 1486, 1356, 1285, 1100, 768, $687 \mathrm{~cm}^{-1} ; \mathbf{R}_{\mathbf{f}} 0.66$ (3:7 EtOAc-hexanes); ${ }^{1} \mathbf{H}$ NMR $\left(500 \mathrm{MHz}, \mathrm{CDCl}_{3}\right) \delta 8.72$ (ddd, $J=4.8,1.8$, $0.9 \mathrm{~Hz}, 1 \mathrm{H}$ ), $7.98(\mathrm{dt}, J=7.9,1.0 \mathrm{~Hz}, 1 \mathrm{H}$ ), 7.81 (td, $J=7.8,1.8 \mathrm{~Hz}, 1 \mathrm{H}$ ), 7.33 (ddd, $J=7.6$, 4.8, $1.1 \mathrm{~Hz}, 1 \mathrm{H}$ ), $7.28-7.21(\mathrm{~m}, 1 \mathrm{H}), 7.15-7.07(\mathrm{~m}, 3 \mathrm{H}$ ), 4.80 (hept, $J=6.0 \mathrm{sz}, 1 \mathrm{H}$ ), 2.72 (ddd, $J=14.8,5.3,3.6 \mathrm{~Hz}, 1 \mathrm{H}$ ), 2.64 (ddd, $J=13.7,10.7,8.1 \mathrm{~Hz}, 1 \mathrm{H}$ ), 2.47 (ddd, $J=13.7,7.8,2.8 \mathrm{~Hz}, 1 \mathrm{H}$ ), 2.19 (ddd, $J=$ $14.9,11.4,5.9 \mathrm{~Hz}, 1 \mathrm{H}), 1.79-1.69(\mathrm{~m}, 1 \mathrm{H}), 1.56-1.46(\mathrm{~m}, 1 \mathrm{H}) ;{ }^{13} \mathrm{C}$ NMR $\left(125 \mathrm{MHz}, \mathrm{CDCl}_{3}\right) \delta 156.4,152.6,149.5,136.7$, 132.4, 129.0, 127.6, 124.8, 123.7, 121.9, 121.4, 106.1, 32.3, 27.9, 20.3; HRMS (ESI) $\mathrm{m} / z$ calcd. for $\mathrm{C}_{18} \mathrm{H}_{16} \mathrm{~F}_{6} \mathrm{NO}_{2}{ }^{+}\left(\mathrm{M}_{+} \mathrm{H}\right)^{+}$ 392.1080; found: 392.1108 .

2-((1,1,1,3,3,3-hexafluoropropan-2-yl)oxy)-2-methyl-3,4,5,6-tetrahydro-2H-benzo[b]oxocine (27). Purified by FCC in

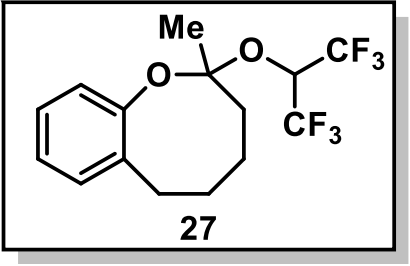
1:19 EtOAc:Hexanes to give $\mathbf{2 7}$ (79 mg, 65\%) as a colorless oil. IR (film) 2935, 2861, 2360 , 2323, 1455, 1358, 1101, 752, $686 \mathrm{~cm}^{-1} ; \mathbf{R}_{\mathbf{f}} 0.75$ (3:7 EtOAc-hexanes); ${ }^{1} \mathbf{H} \mathbf{N M R}(500 \mathrm{MHz}$, $\left.\mathrm{CDCl}_{3}\right) \delta 7.19-7.09(\mathrm{~m}, 3 \mathrm{H}), 6.95(\mathrm{dd}, J=7.7,1.3 \mathrm{~Hz}, 1 \mathrm{H}), 5.04$ (sept, $\left.J=6.0 \mathrm{~Hz}, 1 \mathrm{H}\right), 2.93$ $-2.84(\mathrm{~m}, 1 \mathrm{H}), 2.58(\mathrm{ddd}, J=13.3,6.9,1.6 \mathrm{~Hz}, 1 \mathrm{H}), 2.04-1.93(\mathrm{~m}, 2 \mathrm{H}), 1.89(\mathrm{dd}, J=15.5$, $9.5 \mathrm{~Hz}, 1 \mathrm{H}), 1.61-1.39(\mathrm{~m}, 6 \mathrm{H}) ;{ }^{13} \mathrm{C}$ NMR $\left(125 \mathrm{MHz}, \mathrm{CDCl}_{3}\right) \delta 151.7,138.5,130.8,126.7$, 125.5, 124.1, 108.7, 36.7, 31.4, 31.0, 24.6, 22.0; HRMS (ESI) $\mathrm{m} / \mathrm{z}$ calcd. for $\mathrm{C}_{12} \mathrm{H}_{15} \mathrm{O}^{+}\left[\mathrm{M}-\mathrm{OCH}\left(\mathrm{CF}_{3}\right)_{2}\right]^{+} 175.1123$; found: 175.1132

( \pm )-2-((1,1,1,3,3,3-hexafluoropropan-2-yl)oxy)-2,3-dimethyl-2,3,4,5-tetrahydrobenzo[b]oxepine [( \pm )-28]. Purified by FCC in 1:19 EtOAc:Hexanes to give ( \pm )-28 (110 mg, 87\%) as a colorless oil. IR (film) 2969, 2930, 2360, 2342, 1489, 1358,

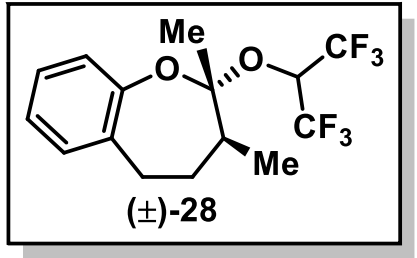
1102, 752, $685 \mathrm{~cm}^{-1} ; \mathbf{R}_{\mathbf{f}} 0.70$ (3:7 EtOAc-hexanes); ${ }^{1} \mathbf{H}$ NMR $\left(500 \mathrm{MHz}, \mathrm{CDCl}_{3}\right) \delta 7.17$ (td, $J=$ 8.0, $2.0 \mathrm{~Hz}, 1 \mathrm{H}), 7.11(\mathrm{dd}, J=7.4,1.7 \mathrm{~Hz}, 1 \mathrm{H}), 7.06(\mathrm{td}, J=7.3,1.2 \mathrm{~Hz}, 1 \mathrm{H}), 6.97(\mathrm{dd}, J=$ 7.9, $0.9 \mathrm{~Hz}, 1 \mathrm{H}$ ), 5.05 (hept, $J=6.1 \mathrm{~Hz}, 1 \mathrm{H}$ ), 2.88 (ddd, $J=14.2,11.3,3.0 \mathrm{~Hz}, 1 \mathrm{H}$ ), 2.67 (ddd, $J=14.3,6.9,2.8 \mathrm{~Hz}, 1 \mathrm{H}), 2.21-2.12(\mathrm{~m}, 1 \mathrm{H}), 1.90-1.82(\mathrm{~m}, 1 \mathrm{H}), 1.36(\mathrm{dtd}, J=14.5,11.7$, $2.8 \mathrm{~Hz}, 1 \mathrm{H}), 1.16(\mathrm{~s}, 3 \mathrm{H}), 1.04(\mathrm{~d}, J=6.8 \mathrm{~Hz}, 3 \mathrm{H}) ;{ }^{13} \mathrm{C} \mathrm{NMR}\left(125 \mathrm{MHz}, \mathrm{CDCl}_{3}\right) \delta 152.6,135.3$, 129.4, 127.2, 124.7, 122.5, 108.1, 31.8, 31.1, 17.4, 17.4; HRMS (ESI) $\mathrm{m} / z$ calcd. for $\mathrm{C}_{12} \mathrm{H}_{15} \mathrm{O}^{+}\left[\mathrm{M}-\mathrm{OCH}\left(\mathrm{CF}_{3}\right)_{2}\right]^{+} 175.1123$; found: 175.1136 . 
2-((1,1,1,3,3,3-hexafluoropropan-2-yl)oxy)-2-methyl-3,4-dihydro-2H-benzo[b][1,4]dioxepine (29). Purified by FCC in

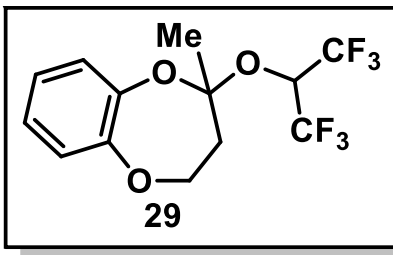

3:7 EtOAc:Hexanes to give 29 (89 mg, 73\%) as a colorless oil. IR (film) 2971, 2890, 2360, 2324, 1494, 1358, 1187, 1097, 760, $687 \mathrm{~cm}^{-1} ; \mathbf{R}_{\mathbf{f}} 0.73$ (4:6 EtOAc-hexanes); ${ }^{1} \mathbf{H}$ NMR (500 $\left.\mathrm{MHz}, \mathrm{CDCl}_{3}\right) \delta 7.06-7.01(\mathrm{~m}, 1 \mathrm{H}), 6.99-6.94(\mathrm{~m}, 3 \mathrm{H}), 4.94$ (hept, $\left.J=6.0 \mathrm{~Hz}, 1 \mathrm{H}\right), 4.48$ (ddd, $J=12.0,6.8,3.4 \mathrm{~Hz}, 1 \mathrm{H}), 4.18-4.11(\mathrm{~m}, 1 \mathrm{H}), 2.42$ (ddd, $J=15.4,8.3,3.3 \mathrm{~Hz}, 1 \mathrm{H}$ ), 2.24 (ddd, $J=15.5,6.8,2.9 \mathrm{~Hz}, 1 \mathrm{H}), 1.59(\mathrm{~s}, 3 \mathrm{H}) ;{ }^{13} \mathrm{C}$ NMR $\left(125 \mathrm{MHz}, \mathrm{CDCl}_{3}\right) \delta$ 149.0, 144.2, 125.3, 123.4, 123.0, 121.5, 105.4, 65.7, 39.1, 23.6; HRMS (ESI) $\mathrm{m} / z$ calcd. for $\mathrm{C}_{10} \mathrm{H}_{11} \mathrm{O}_{2}{ }^{+}\left[\mathrm{M}-\mathrm{OCH}\left(\mathrm{CF}_{3}\right)_{2}\right]^{+}$163.0759; found: 163.0763

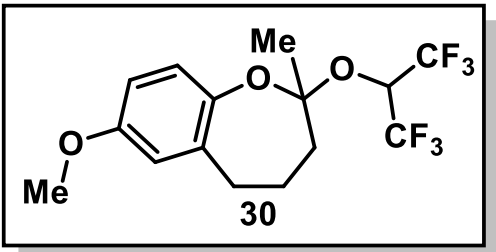

2-((1,1,1,3,3,3-hexafluoropropan-2-yl)oxy)-7-methoxy-2-methyl-2,3,4,5tetrahydrobenzo[b]oxepine (30). Purified by FCC in 3:7 EtOAc:Hexanes to give $\mathbf{3 0}$ (66 mg, 55\%) as a colorless oil.IR (film) 2946, 2835, 2360, 1497, 1359, 1100, 812, 686 $\mathrm{cm}^{-1} ; \mathbf{R}_{\mathbf{f}} 0.58$ (3:7 EtOAc-hexanes); ${ }^{1} \mathbf{H}$ NMR $\left(500 \mathrm{MHz}, \mathrm{CDCl}_{3}\right) \delta 6.88-6.84(\mathrm{~m}, 1 \mathrm{H})$, $6.68-6.64(\mathrm{~m}, 2 \mathrm{H}), 4.99$ (sept, $J=6.0 \mathrm{~Hz}, 1 \mathrm{H}), 3.77(\mathrm{~s}, 3 \mathrm{H}), 2.85-2.77(\mathrm{~m}, 1 \mathrm{H}), 2.65$ (ddd, $J=14.0,6.6,4.3 \mathrm{~Hz}, 1 \mathrm{H}), 2.04-1.89(\mathrm{~m}, 3 \mathrm{H}), 1.66-1.56(\mathrm{~m}, 1 \mathrm{H}), 1.34(\mathrm{~s}, 3 \mathrm{H}) ;{ }^{13} \mathrm{C} \mathrm{NMR}\left(125 \mathrm{MHz}, \mathrm{CDCl}_{3}\right) \delta 156.3$, 146.4, 135.5, 122.6, 115.3, 111.5, 105.6, 69.0, 68.8, 68.5, 68.2, 68.0, 55.4, 38.6, 31.5, 22.6, 21.6; HRMS (ESI) $\mathrm{m} / z$ calcd. for $\mathrm{C}_{12} \mathrm{H}_{15} \mathrm{O}_{2}+\left[\mathrm{M}-\mathrm{OCH}\left(\mathrm{CF}_{3}\right)_{2}\right]^{+}$191.1072; found: 191.1103

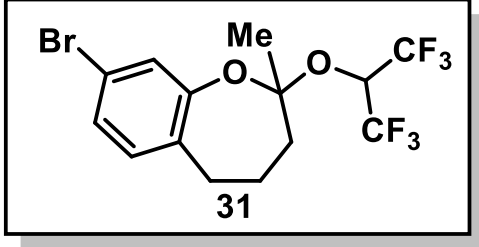

8-bromo-2-((1,1,1,3,3,3-hexafluoropropan-2-yl)oxy)-2-methyl-2,3,4,5tetrahydrobenzo[b]oxepine (31). Purified by FCC in 1:9 EtOAc:Hexanes to give $\mathbf{3 1}$ (116 mg, 75\%) as a colorless oil. IR (film) 2946, 2360, 2342, 1482, 1356, 1098, 1058, $810,686 \mathrm{~cm}^{-1} ; \mathbf{R}_{\mathbf{f}} 0.62$ (3:7 EtOAc-hexanes); ${ }^{1} \mathbf{H}$ NMR $\left(500 \mathrm{MHz}, \mathrm{CDCl}_{3}\right) \delta 7.18$ (dd, $\mathrm{J}=$ 8.0, $2.0 \mathrm{~Hz}, 1 \mathrm{H}$ ), $7.10(\mathrm{~d}, J=2.0 \mathrm{~Hz}, 1 \mathrm{H}), 6.98(\mathrm{~d}, J=8.0 \mathrm{~Hz}, 1 \mathrm{H}), 4.94$ (hept, $J=6.0 \mathrm{~Hz}$, 1H), $2.83-2.75(\mathrm{~m}, 1 \mathrm{H}), 2.67(\mathrm{ddd}, J=14.3,6.2,4.3 \mathrm{~Hz}, 1 \mathrm{H}), 2.01(\mathrm{~m}, 2 \mathrm{H}), 1.99-1.91(\mathrm{~m}, 1 \mathrm{H}), 1.66-1.53(\mathrm{~m}, 1 \mathrm{H}), 1.37$ $(\mathrm{s}, 3 \mathrm{H}) ;{ }^{13} \mathrm{C}$ NMR $\left(125 \mathrm{MHz}, \mathrm{CDCl}_{3}\right) \delta 153.5,133.4,130.9,127.6,125.3,119.8,105.9,38.5,30.9,22.6,21.2$; HRMS (ESI) $\mathrm{m} / \mathrm{z}$ calcd. for $\mathrm{C}_{11} \mathrm{H}_{12} \mathrm{BrO}^{+}\left[\mathrm{M}-\mathrm{OCH}\left(\mathrm{CF}_{3}\right)_{2}\right]^{+} 239.0072$; found: 239.0080 .

2-((1,1,1,3,3,3-hexafluoropropan-2-yl)oxy)-2-methyl-2,3,4,5-tetrahydrobenzo[b]oxepine-8-carbonitrile (32). Purified

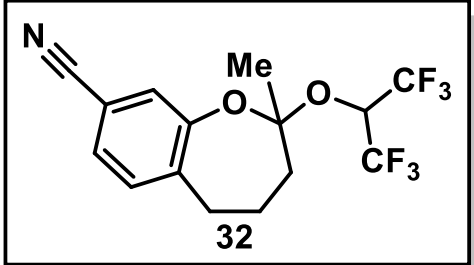

by FCC in 2:8 EtOAc:Hexanes to give 32 (63 mg, 56\%) as a white amorphous solid. IR (neat) 3068, 2980, 2884, 2360, 2342, 2228, 1506, 1362, 1284, 1086, 893, 738, $684 \mathrm{~cm}^{-}$ 1; $\mathbf{R}_{\mathbf{f}} 0.64$ (3:7 EtOAc-hexanes); ${ }^{1} \mathbf{H}$ NMR $\left(500 \mathrm{MHz}, \mathrm{CDCl}_{3}\right) \delta 7.35$ (dd, J = 7.7, $1.6 \mathrm{~Hz}$, 1H), 7.22 (dd, $J=4.6,3.0 \mathrm{~Hz}, 2 \mathrm{H}$ ), 4.93 (sept, $J=6.0 \mathrm{~Hz}, 1 \mathrm{H}), 2.94-2.84(\mathrm{~m}, 1 \mathrm{H}), 2.81$ $-2.74(\mathrm{~m}, 1 \mathrm{H}), 2.05-1.94(\mathrm{~m}, 3 \mathrm{H}), 1.71-1.60(\mathrm{~m}, 1 \mathrm{H}), 1.40(\mathrm{~s}, 3 \mathrm{H}) ;{ }^{13} \mathrm{C} \mathrm{NMR}(125 \mathrm{MHz}$, $\left.\mathrm{CDCl}_{3}\right) \delta 153.1,140.3,130.7,128.5,125.5,118.1,111.2,106.0,106.0,37.7,31.1,22.7,20.6,20.6$; HRMS (ESI) $\mathrm{m} / z$ calcd. for $\mathrm{C}_{12} \mathrm{H}_{12} \mathrm{NO}^{+}\left[\mathrm{M}-\mathrm{OCH}\left(\mathrm{CF}_{3}\right)_{2}\right]^{+}$186.0919; found: 186.0920 . 


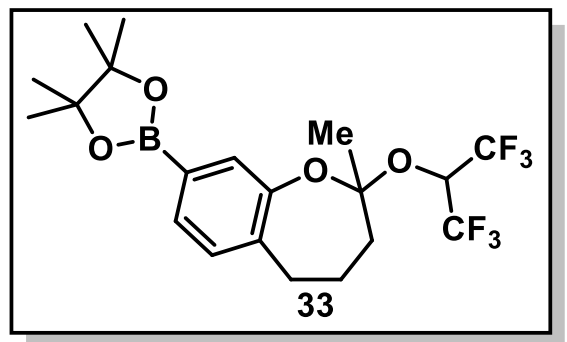

1,3,2-dioxaborolane (33). Purified by FCC in 2:8 EtOAc:Hexanes to give $\mathbf{3 3}$ (122 mg, 72\%) as a colorless oil. IR (film) 2981, 2360, 2342, 1443, 1357, 1178, 1126, 1083, 854, $687 \mathrm{~cm}^{-1} ; \mathbf{R}_{\mathbf{f}} 0.70$ (4:6 EtOAc-hexanes); ${ }^{1} \mathbf{H} \mathbf{N M R}\left(500 \mathrm{MHz}, \mathrm{CDCl}_{3}\right) \delta$ 7.50 (dd, $J=7.3,1.1 \mathrm{~Hz}, 1 \mathrm{H}), 7.33(\mathrm{~d}, J=0.8 \mathrm{~Hz}, 1 \mathrm{H}), 7.12(\mathrm{~d}, J=7.4 \mathrm{~Hz}, 1 \mathrm{H}), 5.04$ (sept, $J=6.0 \mathrm{~Hz}, 1 \mathrm{H}$ ), $2.94-2.84(\mathrm{~m}, 1 \mathrm{H}), 2.69$ (ddd, $J=14.1,5.9,3.5 \mathrm{~Hz}, 1 \mathrm{H}$ ), $2.04(\mathrm{dd}, J=10.0,4.5 \mathrm{~Hz}, 2 \mathrm{H}), 2.02-1.92(\mathrm{~m}, 1 \mathrm{H}), 1.62-1.51(\mathrm{~m}, 1 \mathrm{H}), 1.36(\mathrm{~s}$, $6 \mathrm{H}), 1.35$ (s, 6H), 1.30 (s, 3H); ${ }^{13} \mathrm{C}$ NMR $\left(125 \mathrm{MHz}, \mathrm{CDCl}_{3}\right) \delta 152.4,138.2,131.3$, 129.6, 127.9, 105.6, 83.8, 39.6, 32.1, 24.9, 24.7, 22.5, 21.6.; HRMS (ESI) $m / z$ calcd. for $\mathrm{C}_{20} \mathrm{H}_{25} \mathrm{BF}_{6} \mathrm{NaO}_{4}{ }^{+}(\mathrm{M}+\mathrm{Na})^{+}$477.1678; found: 477.1670 .

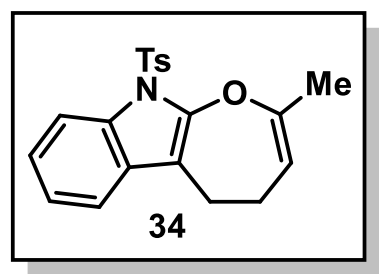

2-methyl-10-tosyl-5,10-dihydro-4H-oxepino[2,3-b]indole (34). Purified by FCC in 2:8 EtOAc:Hexanes to give 34 (113 mg, 86\%) as a pale-yellow solid. Mp: $117-121^{\circ} \mathrm{C}$; IR (neat) 3273, 3067, 2924, 2851, 2162, 1670, 1596, 1368, 1174, 1145, 1083, 934, 813, 753, 665 $\mathrm{cm}^{-1} ; \mathbf{R}_{\mathbf{f}}$ 0.47 (3:7 EtOAc-hexanes); ${ }^{1} \mathbf{H}$ NMR $\left(500 \mathrm{MHz}, \mathrm{CDCl}_{3}\right) \delta 7.98-7.93(\mathrm{~m}, 3 \mathrm{H}), 7.35-7.27$ (m, $3 \mathrm{H}), 7.16(\mathrm{td}, J=7.5,1.0 \mathrm{~Hz}, 1 \mathrm{H}), 7.09-7.06(\mathrm{~m}, 1 \mathrm{H}), 5.72-5.69(\mathrm{~m}, 1 \mathrm{H}), 2.63-2.49(\mathrm{~m}, 2 \mathrm{H})$, 2.46 (ddd, $J=13.2,9.0,5.4 \mathrm{~Hz}, 1 \mathrm{H}), 2.40(\mathrm{~s}, 3 \mathrm{H}), 2.13-2.06(\mathrm{~m}, 1 \mathrm{H}), 1.03(\mathrm{dd}, J=3.7,2.1 \mathrm{~Hz}$, $3 \mathrm{H}) ;{ }^{13} \mathrm{C}$ NMR $\left(125 \mathrm{MHz}, \mathrm{CDCl}_{3}\right) \delta 178.4,145.4,139.5,138.6,135.2,132.2,130.7,129.6,128.5,127.8,125.1,123.3,113.4$, 63.7, 37.1, 31.3, 21.6, 12.5; HRMS (ESI) $\mathrm{m} / z$ calcd. for $\mathrm{C}_{20} \mathrm{H}_{19} \mathrm{NNaO}_{3} \mathrm{~S}^{+}(\mathrm{M}+\mathrm{Na})^{+}$376.0978; found: 376.1009

\section{Derivatization of HFIP-Acetals}

NOTE: The low yields are likely due to the high volatility of the hydrocarbon product. The reactions produce no detectable byproducts and full conversion as detected by GC-MS.

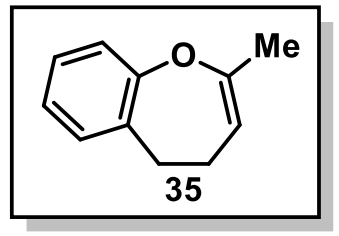

2-methyl-4,5-dihydrobenzo[b]oxepine (35). Acetal $11(52.7 \mathrm{mg}, 0.161 \mathrm{mmol})$ and pyridinium $\mathrm{p}$ toluenesulfonate $(44.3 \mathrm{mg}, 0.176 \mathrm{mmol})$ were added to a flame-dried sealed test tube under argon at room temperature and dissolved in benzene $(0.80 \mathrm{~mL})$. Anhydrous pyridine $(10.0 \mu \mathrm{L}, 0.124$ $\mathrm{mmol}$ ) was added and the reaction was heated to $120^{\circ} \mathrm{C}$ overnight. After the reaction was complete by TLC, the reaction was concentrated by rotary evaporation and purified by flash column chromatography $\left(100 \% \rightarrow 1: 50\right.$ unstabilized $\mathrm{Et}_{2} \mathrm{O}$ :pentane) to give the desired product as a clear oil (13.9 $\left.\mathrm{mg}, 54 \%\right)$. $\mathbf{R}_{\boldsymbol{f}}$ 0.70 (9:1 hexanes-EtOAc); IR (film) 3037, 2947, 2917, 2846, 2360, 2341, 1686, 1488, 1381, 1237, 1105, 756, $688 \mathrm{~cm}^{-1} ;{ }^{1} \mathbf{H}$ NMR $\left(500 \mathrm{MHz}, \mathrm{C}_{6} \mathrm{D}_{6}\right) \delta 7.07-7.04(\mathrm{~m}, 1 \mathrm{H}), 6.98-6.94(\mathrm{~m}, 1 \mathrm{H}), 6.90-6.84(\mathrm{~m}, 2 \mathrm{H}), 4.47(\mathrm{t}, J=4.3 \mathrm{~Hz}, 1 \mathrm{H}), 2.80(\mathrm{t}, J=$ $6.0 \mathrm{~Hz}, 2 \mathrm{H}), 2.08-2.03(\mathrm{~m}, 2 \mathrm{H}), 1.86-1.84(\mathrm{~m}, 3 \mathrm{H}) ;{ }^{13} \mathrm{C} \mathbf{N M R}\left(125 \mathrm{MHz}, \mathrm{CDCl}_{3}\right) \delta 157.9,151.1,134.2,129.2,126.8$, 123.8, 120.2, 103.9, 31.2, 27.3, 22.7; HRMS (ESI) m/z calcd. for $\mathrm{C}_{11} \mathrm{H}_{13} \mathrm{O}^{+}(\mathrm{M}-\mathrm{H})^{+}$161.0966; found: 161.0959 .

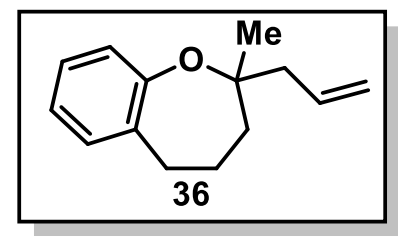

2-allyl-2-methyl-2,3,4,5-tetrahydrobenzo[b]oxepine (36). Acetal 11 (58 mg, $0.192 \mathrm{mmol}$ ) was dissolved in dry $\mathrm{CH}_{2} \mathrm{Cl}_{2}(884 \mu \mathrm{L})$, in a flame-dried vial under argon. The solution was cooled to $-78{ }^{\circ} \mathrm{C}$ then allyltrimethylsilane $(140 \mu \mathrm{L}, 0.884 \mathrm{mmol})$ and $\mathrm{HBF}_{4} \cdot \mathrm{OEt}_{2}(1 \mathrm{drop}, 0.0177$ mmol; $50 \%-55 \%$ complex) were added sequentially. The reaction was slowly warmed to r.t. and monitored by TLC. Once complete, the reaction was quenched with $884 \mu \mathrm{L}$ of $\mathrm{MeOH}$. The solution was concentrated and purified by column chromatography in 1:19 diethyl ether:pentane to give the product (16 mg, 
44\%) as a colorless oil. IR (film) 3073, 2978, 2928, 2859, 2360, 2342, 1639, 1487, 1377, 1074, 761, 734, $667 \mathrm{~cm}^{-1} ; \mathbf{R}_{\mathbf{f}} 0.79$ (1:9 Et $2 \mathrm{O}$ :pentane); ${ }^{1} \mathbf{H}$ NMR $\left(500 \mathrm{MHz}, \mathrm{CDCl}_{3}\right) \delta 7.13-7.06(\mathrm{~m}, 2 \mathrm{H}), 6.97(\mathrm{td}, J=7.4,1.3 \mathrm{~Hz}, 1 \mathrm{H}), 6.90(\mathrm{dd}, J=7.8,1.0$ $\mathrm{Hz}, 1 \mathrm{H}$ ), 5.94 (dddd, $J=17.0,10.3,7.8,6.8 \mathrm{~Hz}, 1 \mathrm{H}), 5.14-5.04(\mathrm{~m}, 2 \mathrm{H}), 2.84-2.69(\mathrm{~m}, 1 \mathrm{H}), 2.41(\mathrm{dd}, J=13.8,6.8 \mathrm{~Hz}$, $1 \mathrm{H}), 2.30(\mathrm{dd}, J=13.7,7.8 \mathrm{~Hz}, 1 \mathrm{H}), 1.89-1.80(\mathrm{~m}, 1 \mathrm{H}), 1.79-1.62(\mathrm{~m}, 3 \mathrm{H}), 1.12(\mathrm{~s}, 3 \mathrm{H}) ;{ }^{13} \mathrm{C} \mathrm{NMR}\left(125 \mathrm{MHz}, \mathrm{CDCl}_{3}\right) \delta$ 154.8, 135.4, 129.9, 127.7, 125.3, 123.0, 118.6, 75.8, 41.7, 32.8, 28.4, 23.0; HRMS (ESI) $\mathrm{m} / \mathrm{z}$ calcd. for $\mathrm{C}_{14} \mathrm{H}_{19} \mathrm{O}^{+}(\mathrm{M}+\mathrm{H})^{+}$ 203.1436; found: 203.1428 .

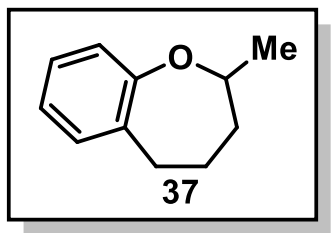

2-methyl-2,3,4,5-tetrahydrobenzo[b]oxepine (37). Acetal 11 (26 mg, $0.0801 \mathrm{mmol}$ ) was dissolved in dry $\mathrm{CH}_{2} \mathrm{Cl}_{2}(401 \mu \mathrm{L})$, in a flame-dried vial under argon. The solution was cooled to -78 ${ }^{\circ} \mathrm{C}$ then Triethylsilane $\left(64 \mu \mathrm{L}, 0.401 \mathrm{mmol}\right.$ ) and $\mathrm{HBF}_{4} \cdot \mathrm{OEt}_{2}\left(1 \mathrm{drop}, 8.01 \times 10^{-3} \mathrm{mmol} ; 50 \%-55 \%\right.$ complex) were added sequentially. The reaction was slowly warmed to $-20{ }^{\circ} \mathrm{C}$ and monitored by GCMS. Once complete, the reaction was quenched with $401 \mu \mathrm{L}$ of $\mathrm{MeOH}$. The solution was concentrated and purified by column chromatography in 1:19 diethyl ether:pentane to give the product (7 mg, $54 \%)$ as a colorless oil. IR (film) 2972, 2927, 2360, 2342, 1487, 1376, 1065, 730, $648 \mathrm{~cm}^{-1} ; \mathbf{R}_{\mathbf{f}} 0.73$ (1:9 Et $2 \mathrm{O}$ :pentane); ${ }^{1} \mathbf{H}$ NMR (500 $\left.\mathrm{MHz}, \mathrm{CDCl}_{3}\right) \delta 7.15-7.08(\mathrm{~m}, 2 \mathrm{H}), 7.00-6.94(\mathrm{~m}, 2 \mathrm{H}), 3.81-3.72(\mathrm{~m}, 1 \mathrm{H}), 2.94-2.84(\mathrm{~m}, 1 \mathrm{H}), 2.75-2.66(\mathrm{~m}, 1 \mathrm{H}), 2.00$

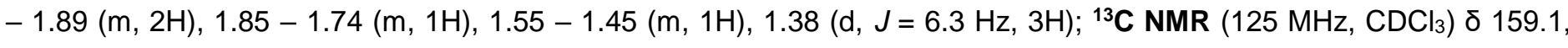
135.9, 130.0, 127.2, 123.3, 121.5, 79.4, 77.2, 77.0, 76.7, 39.0, 33.8, 25.8, 23.0; HRMS (ESI) $\mathrm{m} / z$ calcd. for $\mathrm{C}_{11} \mathrm{H}_{13} \mathrm{O}^{+}(\mathrm{M}-\mathrm{H})^{+}$ 161.0966; found: 161.0959 .

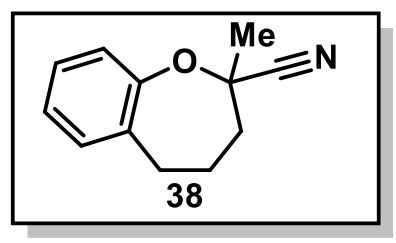

2-methyl-2,3,4,5-tetrahydrobenzo[b]oxepine-2-carbonitrile (38). Acetal 11 (58 mg, 0.177 mmol) was dissolved in dry $\mathrm{CH}_{2} \mathrm{Cl}_{2}(884 \mu \mathrm{L})$, in a flame-dried vial under argon. The solution was cooled to $-78{ }^{\circ} \mathrm{C}$ then Trimethylsilyl cyanide $(111 \mu \mathrm{L}, 0.884 \mathrm{mmol})$ and $\mathrm{HBF}_{4} \cdot \mathrm{OEt}_{2}(42 \mu \mathrm{L}$, $0.177 \mathrm{mmol} ; 50 \%$ - 55\% complex) were added sequentially. The reaction was slowly warmed to $0{ }^{\circ} \mathrm{C}$ and monitored by TLC. Once complete, the reaction was quenched with $884 \mu \mathrm{L}$ of $\mathrm{MeOH}$. The solution was concentrated and purified by column chromatography in 2:8 EtOAc:pentane to give the product (24 mg, 71\%) as a colorless oil. IR (film) 2939, 2862, 2360, 2342, 2254, 1488, 1379, 1081, 727, $649 \mathrm{~cm}^{-1} ; \mathbf{R}_{\mathbf{f}} 0.55(2: 8$ EtOAc:hexanes); ${ }^{1} \mathbf{H}$ NMR $\left(500 \mathrm{MHz}, \mathrm{CDCl}_{3}\right) \delta 7.23-7.18(\mathrm{~m}, 1 \mathrm{H}), 7.16-7.13(\mathrm{~m}, 1 \mathrm{H}), 7.11-7.07(\mathrm{~m}, 2 \mathrm{H}), 2.87$ (ddd, J $=14.4,11.7,2.8 \mathrm{~Hz}, 1 \mathrm{H}), 2.75(\mathrm{ddd}, J=14.2,5.9,2.2 \mathrm{~Hz}, 1 \mathrm{H}), 2.09-2.02(\mathrm{~m}, 1 \mathrm{H}), 2.02-1.95(\mathrm{~m}, 1 \mathrm{H}), 1.82(\mathrm{~s}, 3 \mathrm{H}), 1.80$ $-1.73(\mathrm{~m}, 1 \mathrm{H}) ;{ }^{13} \mathrm{C}$ NMR $\left(125 \mathrm{MHz}, \mathrm{CDCl}_{3}\right) \delta 154.8,135.4,129.9,127.7,125.3,123.0,118.6,75.8,41.7,32.8,28.4,23.0$; HRMS (ESI) $\mathrm{m} / z$ calcd. for $\mathrm{C}_{11} \mathrm{H}_{13} \mathrm{O}^{+}(\mathrm{M}-\mathrm{CN})+161.0966$; found: 161.0957.

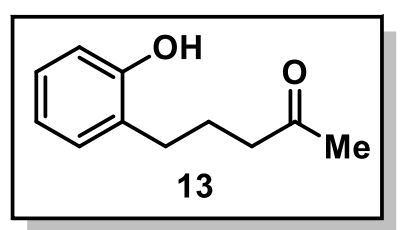

5-(2-hydroxyphenyl)pentan-2-one (13). HVI reagent 14 (409 mg, $0.620 \mathrm{mmol}$ ) was added to a flame dried vial and suspended in dry $\mathrm{CH}_{2} \mathrm{Cl}_{2}(1.05 \mathrm{~mL})$ under argon. Alcohol 7 (67 mg, 0.413 $\mathrm{mmol})$, dissolved in dry $\mathrm{CH}_{2} \mathrm{Cl}_{2}(1.05 \mathrm{~mL})$, was added to the suspension at r.t. and the reaction was monitered by TLC. Once complete, the reaction was concentrated and purified by column chromatography in 1:9 EtOAc-hexanes $\rightarrow$ 3:7 EtOAc-hexanes to give ketone $\mathbf{1 3}$ (25 $\mathrm{mg}, 33 \%$ ) as a pale yellow oil. IR (film) 3234, 2948, 2892, 2323, 1692, 1596, 1455, 1355, 1228, 753, $694 \mathrm{~cm}^{-1} ; \mathbf{R}_{\mathbf{f}} 0.28$ (3:7 EtOAc:hexanes); ${ }^{1} \mathbf{H} \mathbf{~ N M R}(500 \mathrm{MHz}$, $\left.\mathrm{CDCl}_{3}\right) \delta 7.11(\mathrm{td}, J=7.9,1.6 \mathrm{~Hz}, 1 \mathrm{H}), 7.08-7.03(\mathrm{~m}, 1 \mathrm{H}), 6.89(\mathrm{~d}, J=8.0 \mathrm{~Hz}, 1 \mathrm{H}), 6.82(\mathrm{t}, J=7.4 \mathrm{~Hz}, 1 \mathrm{H}), 3.20(\mathrm{~s}, 1 \mathrm{H})$, $2.56(\mathrm{dd}, J=10.5,4.1 \mathrm{~Hz}, 4 \mathrm{H}), 2.20(\mathrm{~s}, 3 \mathrm{H}), 1.87-1.80(\mathrm{~m}, 2 \mathrm{H}) ;{ }^{13} \mathrm{C}$ NMR $\left(125 \mathrm{MHz}, \mathrm{CDCl}_{3}\right) \delta 210.9,154.5,129.8,127.5$, 127.1, 120.0, 115.8, 42.2, 29.9, 29.4, 23.6; HRMS (ESI) $\mathrm{m} / z$ calcd. for $\mathrm{C}_{11} \mathrm{H}_{14} \mathrm{NaO}^{+}(\mathrm{M}+\mathrm{Na})^{+} 201.0891$; found: 201.0888. 


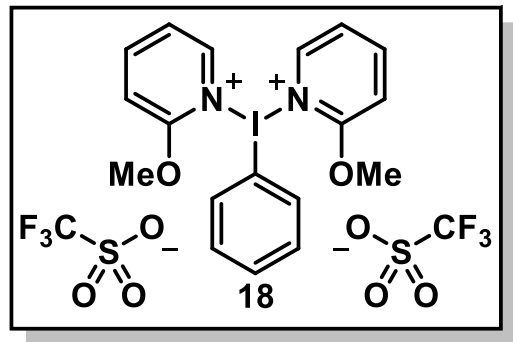

1,1'-(phenyl- $\lambda^{3}$-iodanediyl)bis(2-methoxypyridin-1-ium)

trifluoromethanesulfonate (18). PIDA (2.01 g, $6.24 \mathrm{mmol})$ was placed in a flame-dried flask under argon and dissolved in dry $\mathrm{CH}_{2} \mathrm{Cl}_{2}(62 \mathrm{~mL})$. Trimethylsilyl trifluoromethanesulfonate $(2.26 \mathrm{~mL}, 12.5 \mathrm{mmol}$, neat) and 2-methoxypyridine $(1.31 \mathrm{~mL}$, $12.5 \mathrm{mmol})$ in a solution of $\mathrm{CH}_{2} \mathrm{Cl}_{2}(25 \mathrm{~mL})$ was added sequentially at room temperature and the reaction was allowed to stir of 30 mins. The white solid was then quickly filtered and washed with dry $\mathrm{CH}_{2} \mathrm{Cl}_{2}(3 \times 15 \mathrm{~mL})$ and placed into a flame-dried vial. The 2-methoxy $\mathrm{HVI}$ reagent 18 (2.94 g, 65\%) was isolated as a white solid. The compound was characterized by $\mathrm{x}$-ray analysis, but no melting point were determined due to the hygroscopic nature of the reagent.

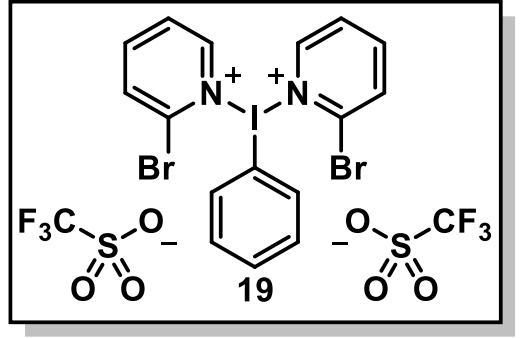

1,1'-(phenyl- $\lambda^{3}$-iodanediyl)bis(2-bromopyridin-1-ium) trifluoromethanesulfonate (19). PIDA (1.77 g, $5.50 \mathrm{mmol}$ ) was placed in a flame-dried flask under argon and dissolved in dry $\mathrm{CH}_{2} \mathrm{Cl}_{2}(55 \mathrm{~mL})$. Trimethylsilyl trifluoromethanesulfonate (1.99 mL, 11.0 mmol, neat) and 2-bromopyridine $(1.05 \mathrm{~mL}, 11.0 \mathrm{mmol})$ in a solution of $\mathrm{CH}_{2} \mathrm{Cl}_{2}(22 \mathrm{~mL})$ was added sequentially at room temperature and the reaction was allowed to stir of 30 mins. The white solid was then quickly filtered and washed with dry $\mathrm{CH}_{2} \mathrm{Cl}_{2}(3 \times 15 \mathrm{~mL})$ and placed into a flame-dried vial. The 2-bromo HVI reagent 19 (2.47 g, 55\%) was isolated as a white solid. IR (neat) 3091, 3063, 3010, 1603, 1422, 1223, 1052, 1025, 1012, 783, $641 \mathrm{~cm}^{-1} ;{ }^{1} \mathbf{H}$ NMR [500 MHz, CDCl $:$ TFA (20:1)] $\delta 8.93$ (dd, $J=5.8$, $1.4 \mathrm{~Hz}, 2 \mathrm{H}$ ), 8.33 (td, $J=10.0,2.0 \mathrm{~Hz}, 2 \mathrm{H}), 8.21-8.18(\mathrm{~m}, 1 \mathrm{H}), 8.07$ (d, J = 8.3 Hz, 2H), 7.97 (ddd, J = 7.6, 5.9, 1.1 Hz, $2 \mathrm{H}), 7.75(\mathrm{t}, J=7.5 \mathrm{~Hz}, 1 \mathrm{H}), 7.62(\mathrm{t}, J=7.9 \mathrm{~Hz}, 1 \mathrm{H}) ;{ }^{13} \mathrm{C}$ NMR [125 MHz, CDCl 3 :TFA (20:1)] $\delta$ 147.6, 144.9, 135.1, 134.4, 133.7, 132.0, 132.0, 126.0, 122.7; HRMS (ESI) $\mathrm{m} / \mathrm{z}$ calcd. for $\mathrm{C}_{6} \mathrm{H}_{5} \mathrm{I}^{+}\left[\mathrm{M}-\left(\mathrm{C}_{5} \mathrm{H}_{4} \mathrm{BrN}\right)_{2}-\left(\mathrm{CF}_{3} \mathrm{O}_{3} \mathrm{~S}\right)_{2}\right]^{+}$203.9436; found: 203.9451.

\section{References}

(1) Noji, M.; Ohno, T.; Fuji, K.; Futaba, N.; Tajima, H.; Ishii, K. J. Org. Chem. 2003, 68, 9340-9347.

(2) Hatano, M.; Ito, O.; Suzuki, S.; Ishihara, K. J. Org. Chem. 2010, 75, 5008-5016.

(3) Nakamura, K.; Hatajima, T.; Kamiya, Y. J. Am. Chem. Soc. 1989, 111, 4392-4398.

(4) Taniguchi, T.; Zaimoku, H.; Ishibashi, H. Chem. Eur. J. 2011, 17, 4307-4312.

(5) Jones, P.; Knochel, P. J. Org. Chem. 1999, 64, 186-195.

(6) McAdam, C. A.; McLaughlin, M. G.; Johnston, A. J. S.; Chen, J.; Walter, M. W.; Cook, M. J. Org. Biomol. Chem. 2013, 11, 4488-4502.

(7) Hatano, M.; Miyamoto, T.; Ishihara, K. Org. Lett. 2007, 9, 4535-4538.

(8) Nicolaou, K. C.; Yu, R.; Shi, L.; Cai, Q.; Lu, M.; Heretsch, P. Org. Lett., 2013, 15, 1994-1997. 


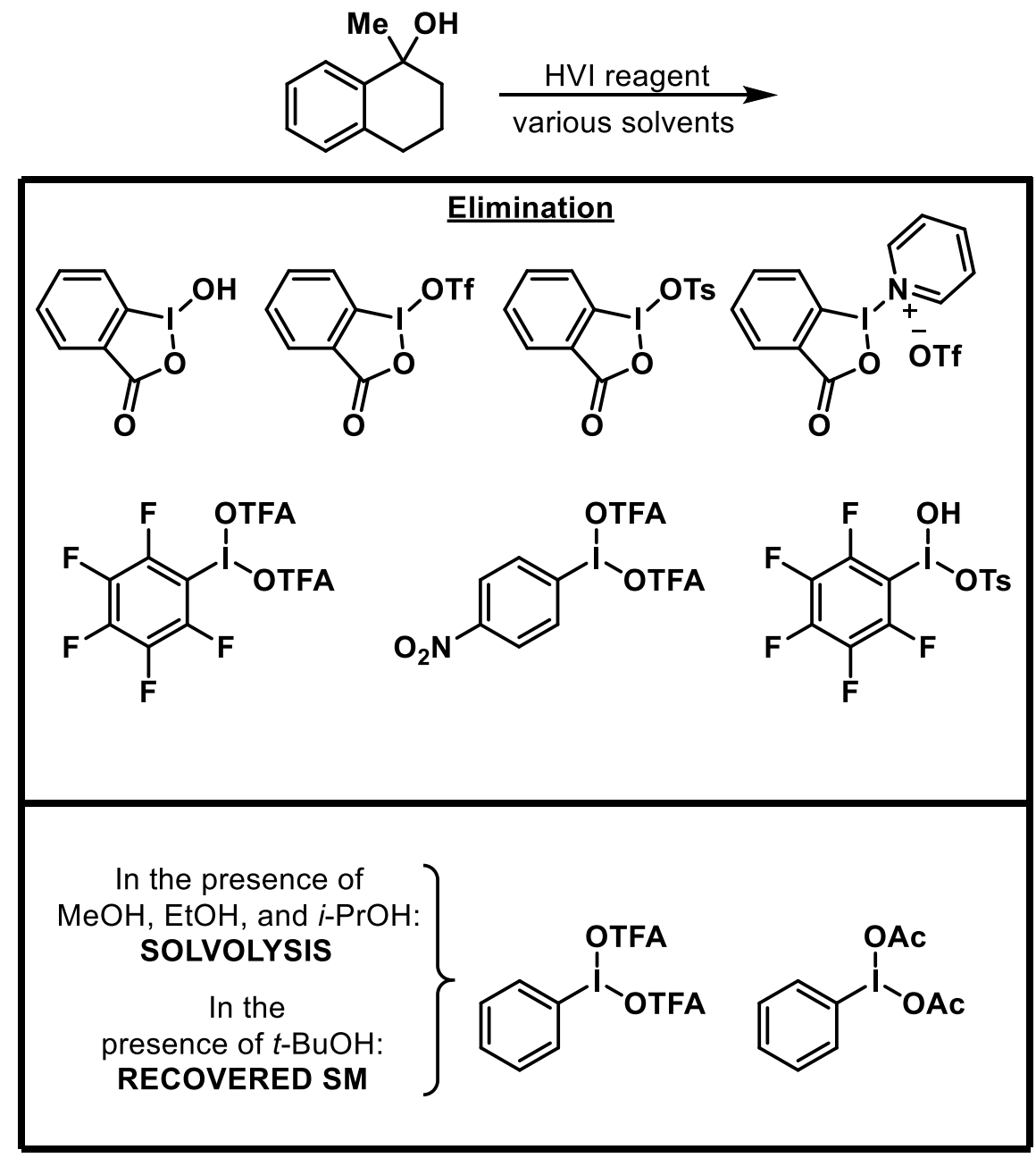



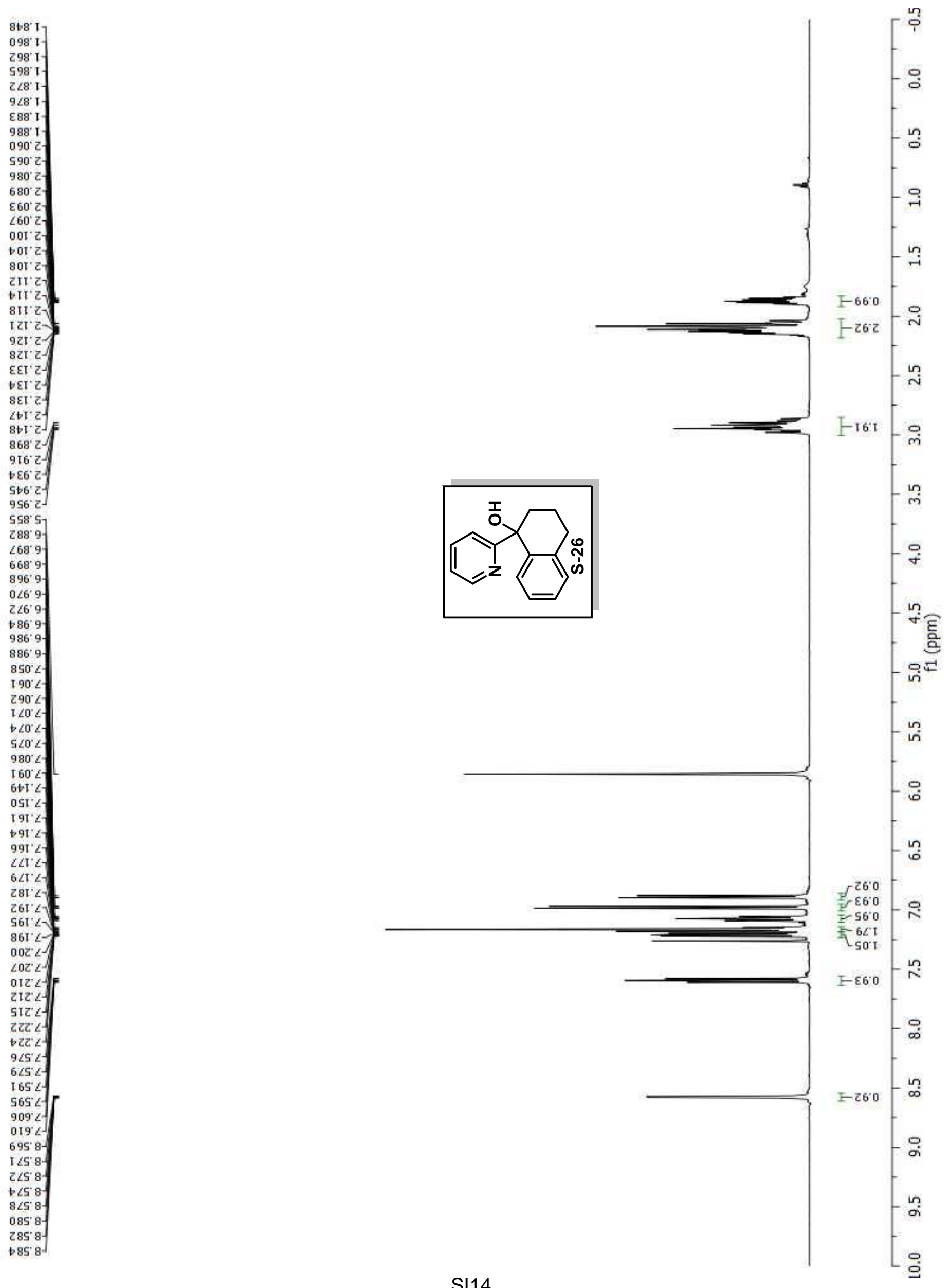


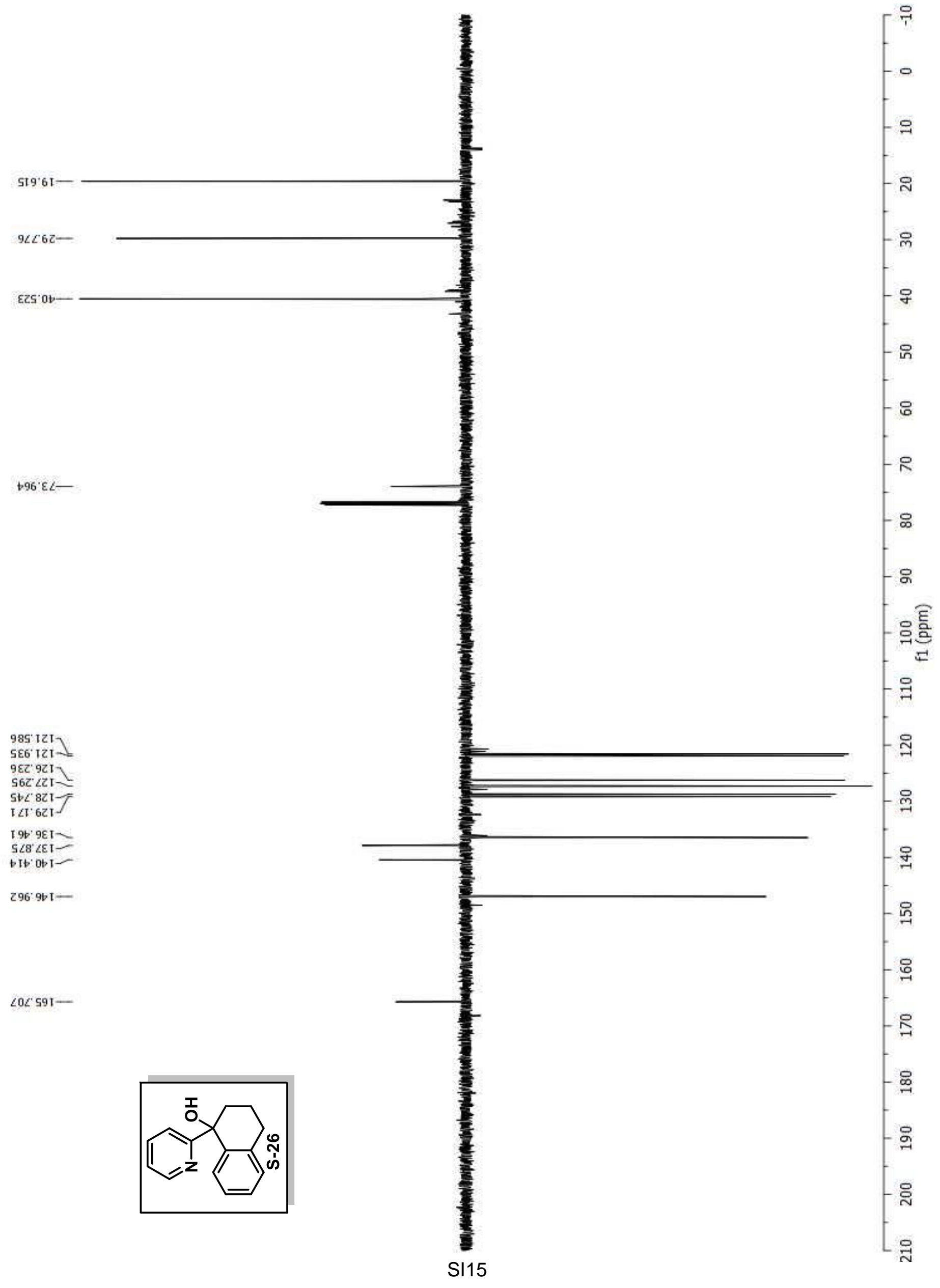




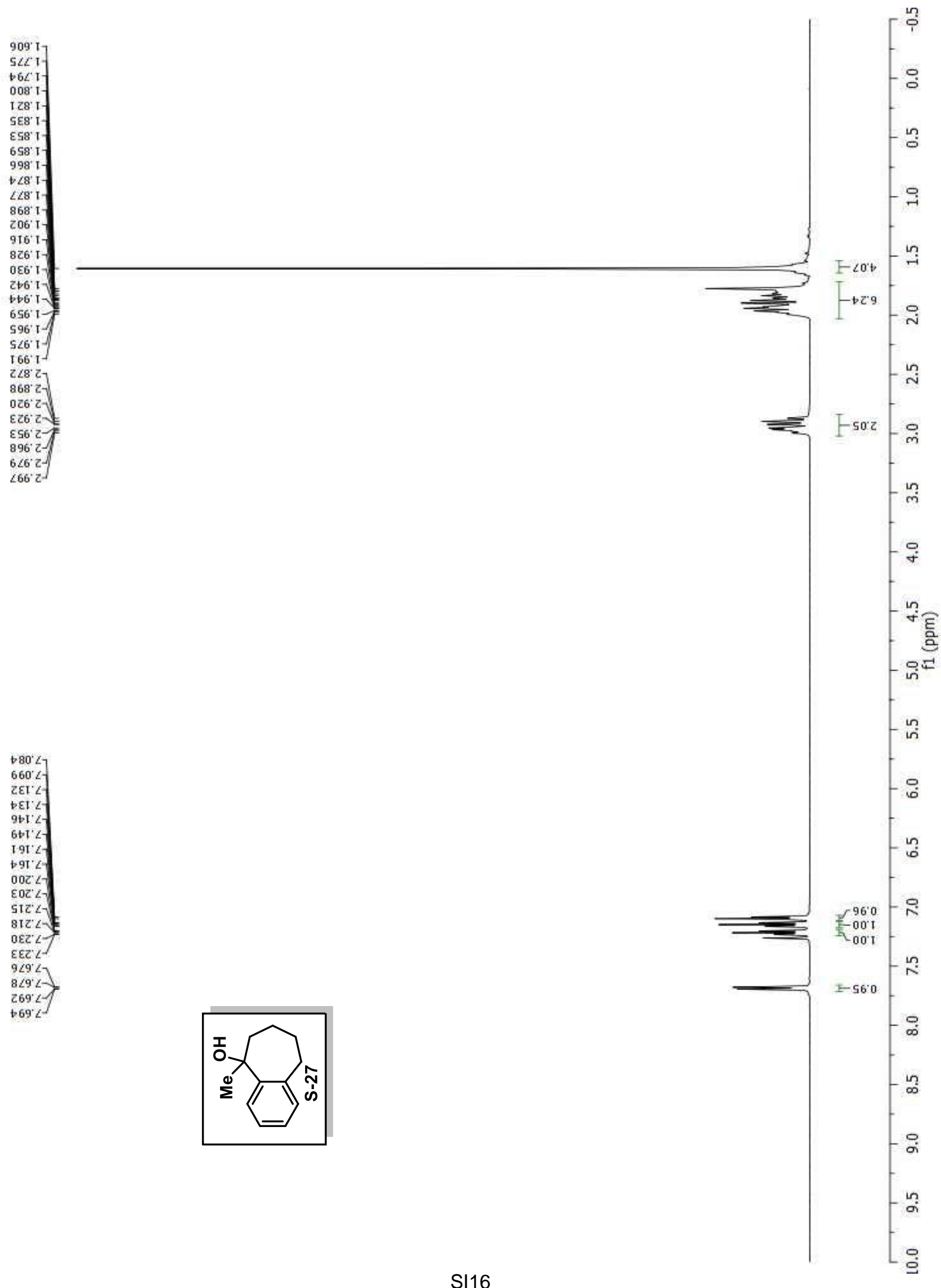




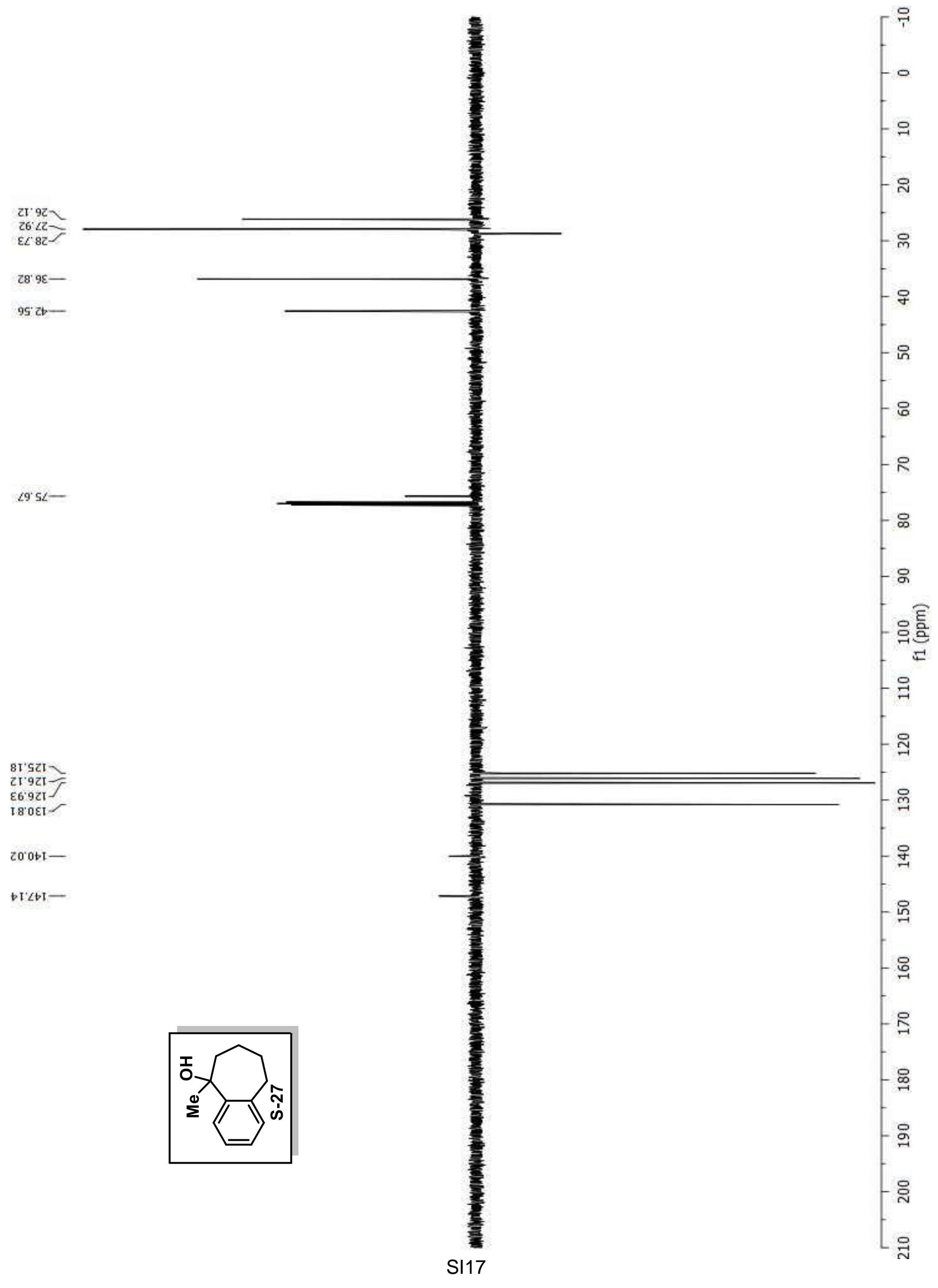




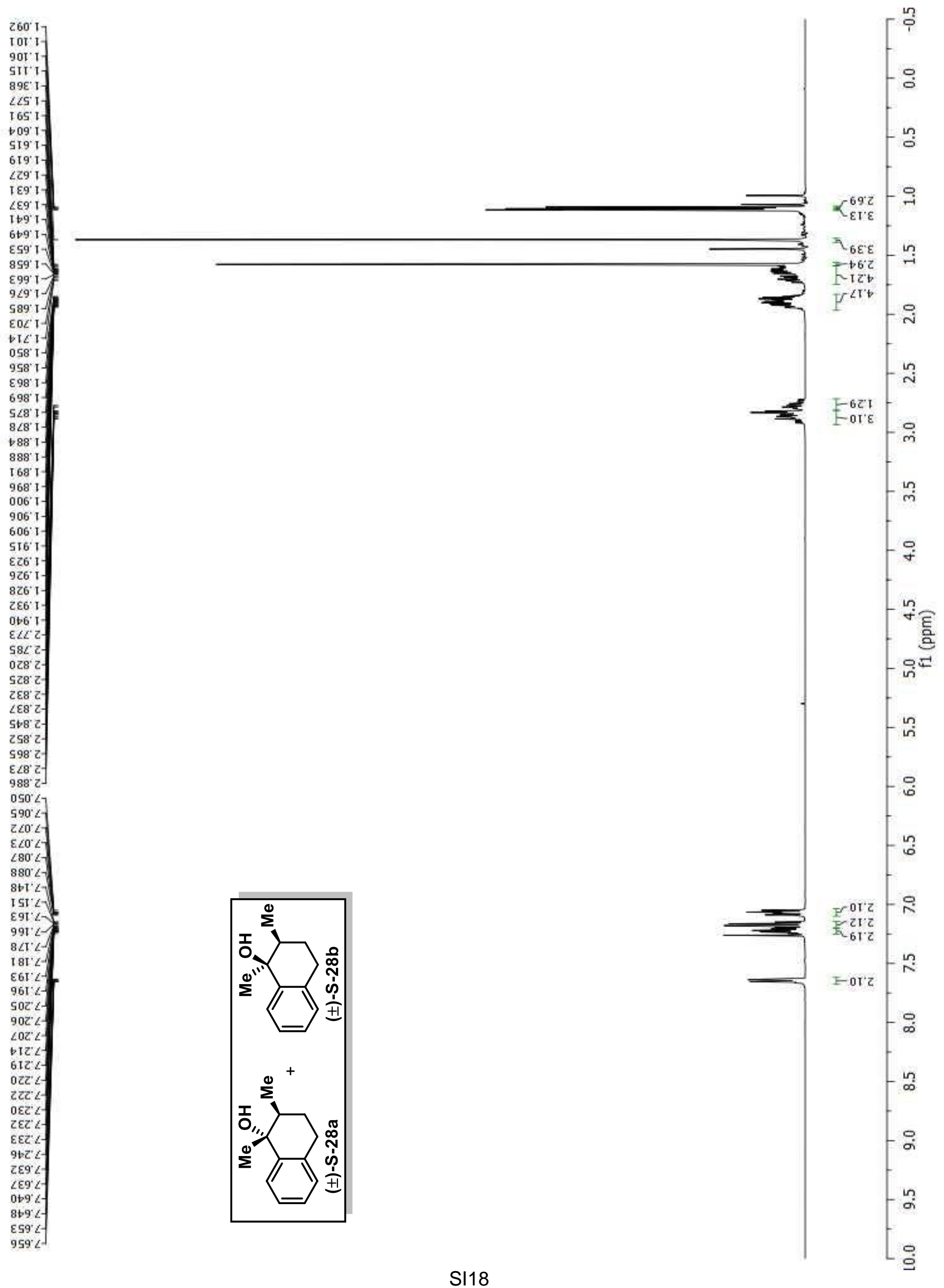


${ }_{0.51}=$

$18+27$

$0 z: 2]$

$6 \subset \angle 27$

$0582-$

$\left.\begin{array}{c}28 \cdot 8 z- \\ \varepsilon \varepsilon \cdot 6 z\end{array}\right]$

9E: $6 \varepsilon-$

$60 \mathrm{It}-$

I $2: 2$

to $\varepsilon L=$

EI $92 \mathrm{II}$

62 921

ZE 9 ZI-

$6<92 \mathrm{I}$

I 8 वृा

16.921 -

$t+821-7$

$69.821-$

ย9'SEI >

$2 t=2 t-$

$99+6 \mathrm{I}-$
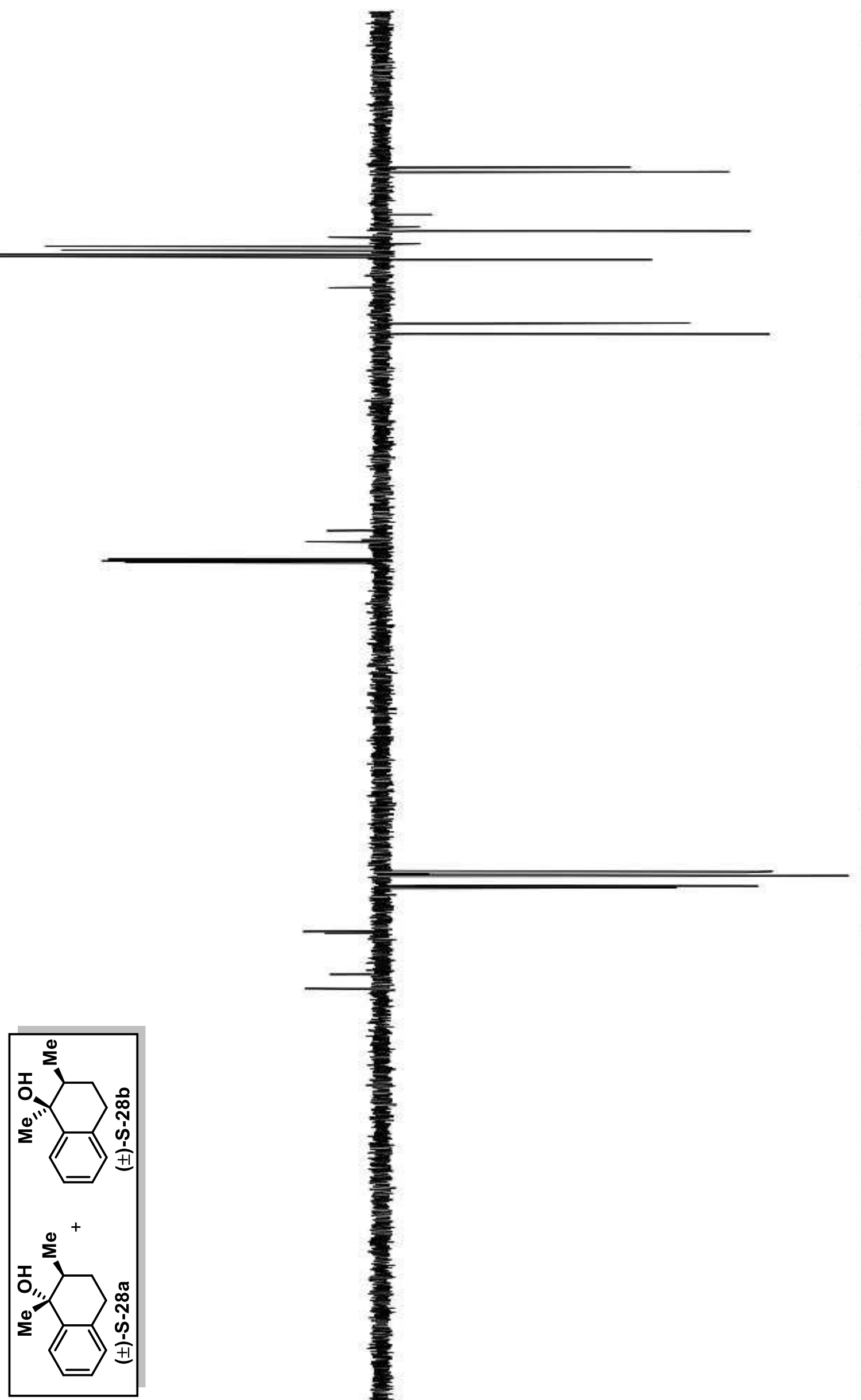


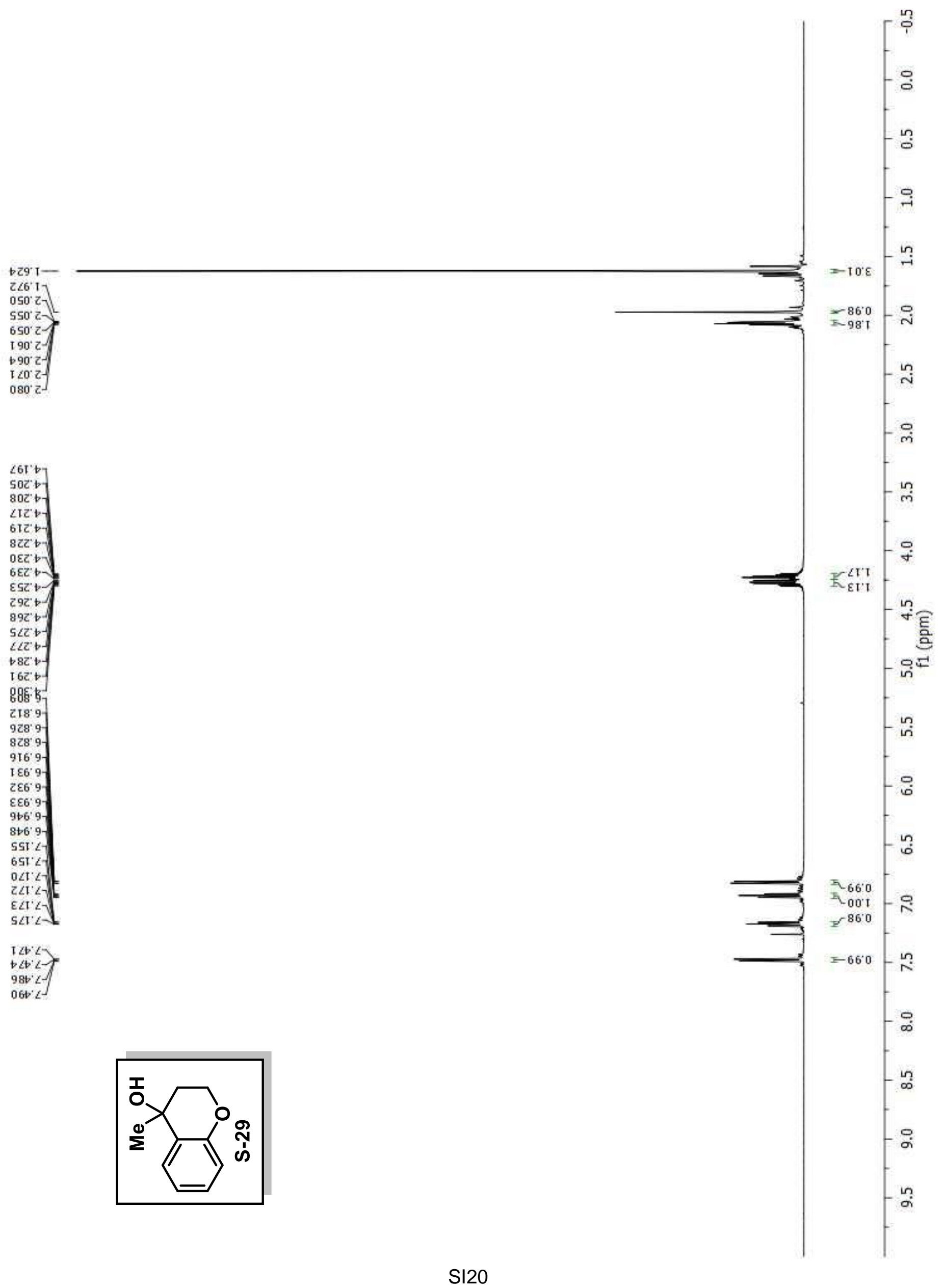




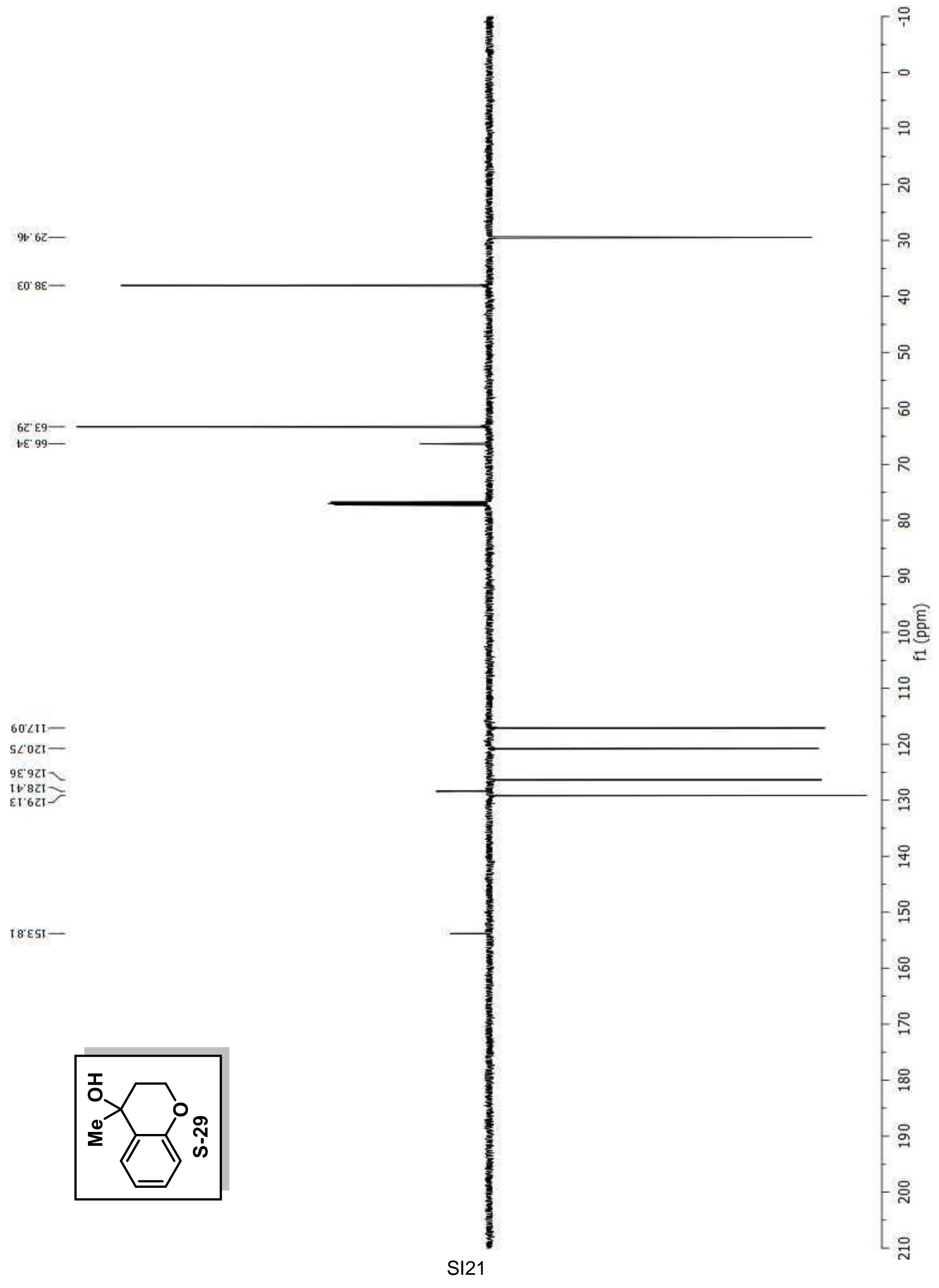




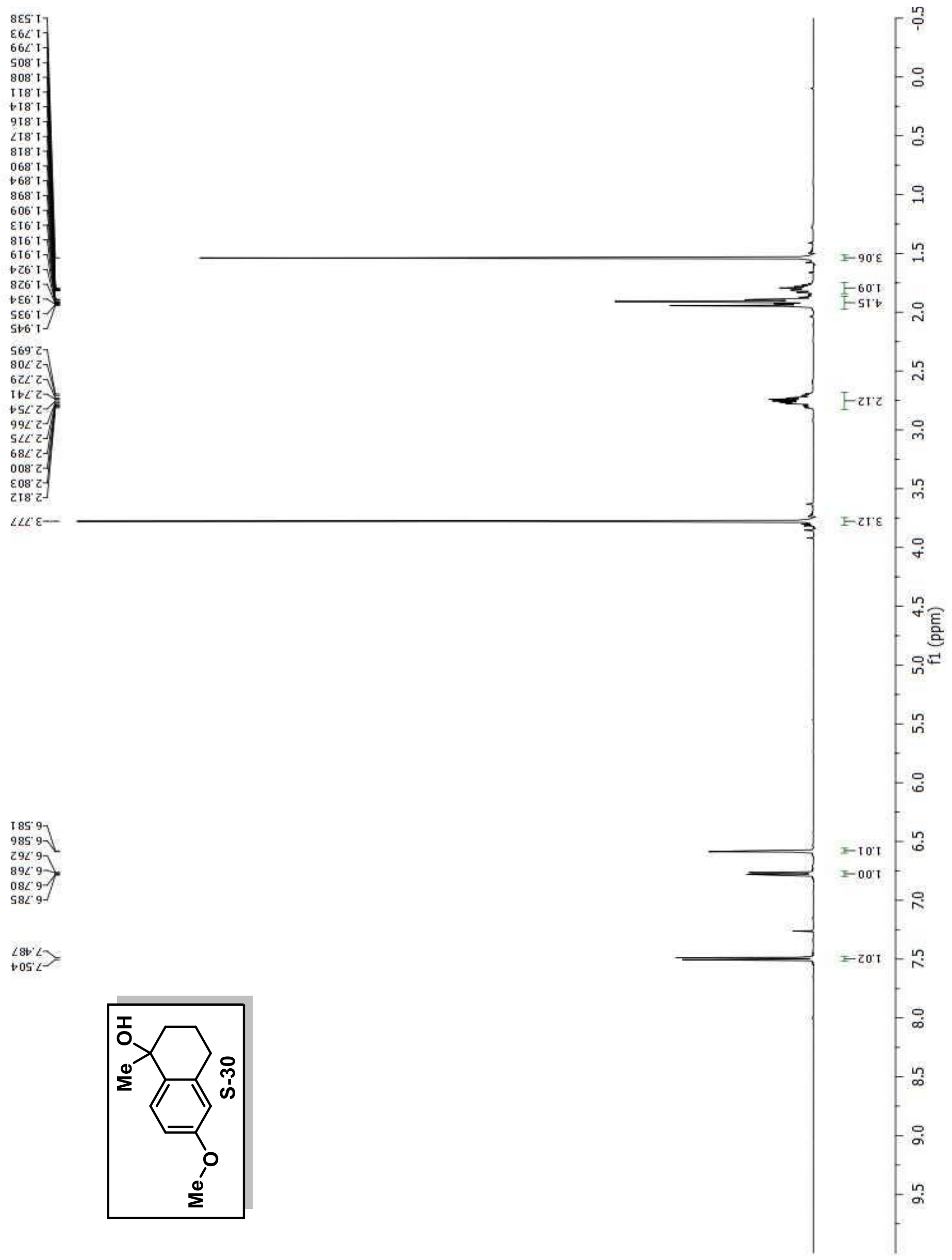


$1 \varepsilon \cdot 0 z-$

$5 z^{\circ} 0 \varepsilon$
$29^{\circ} 0 \varepsilon$

I8 $6 \varepsilon-$

90'ss-

OI $02-$

8s: 211
98211

$85 \angle 21-$

6I SEI$0<\angle \varepsilon I-$

8Z' $851-$
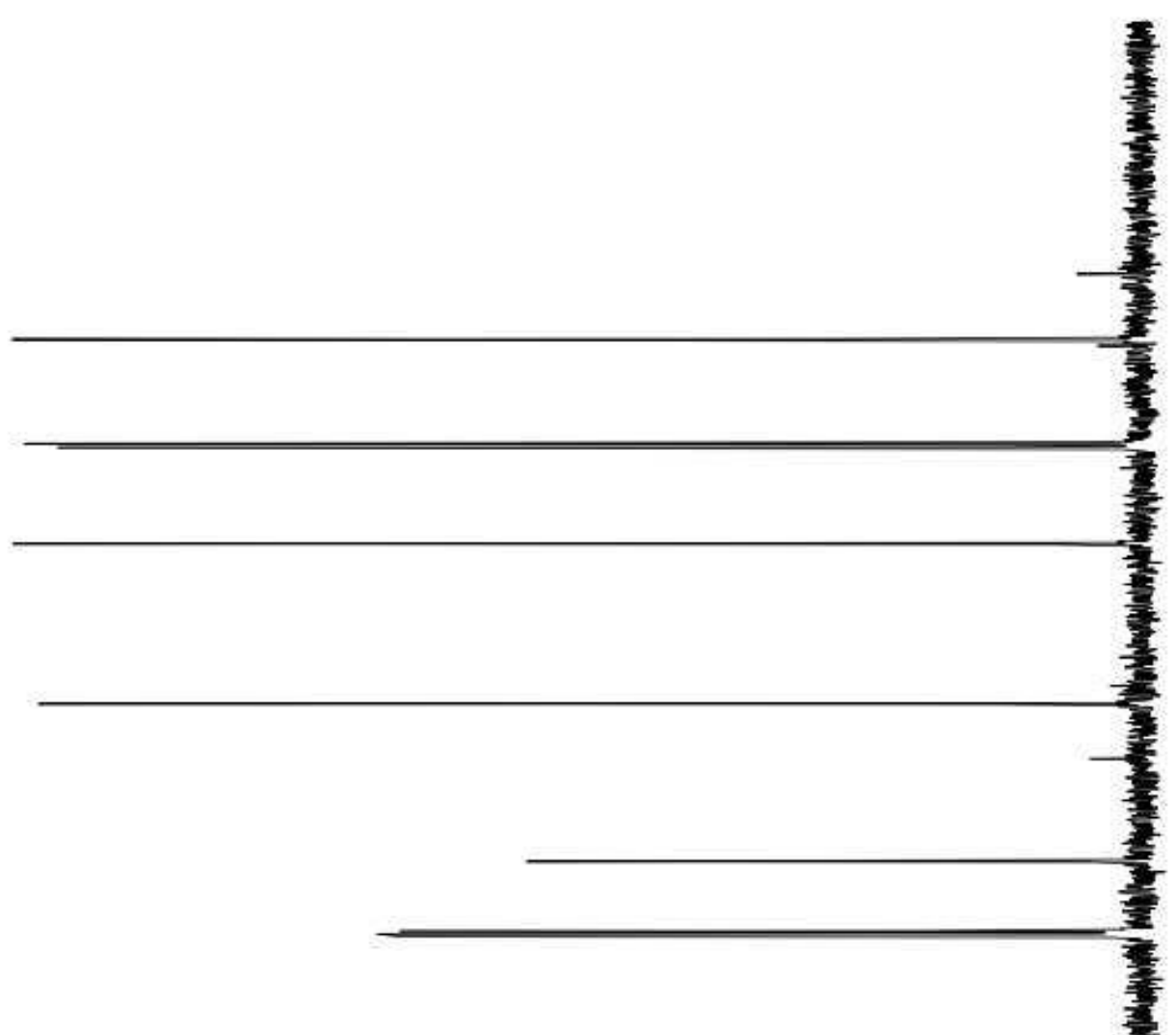


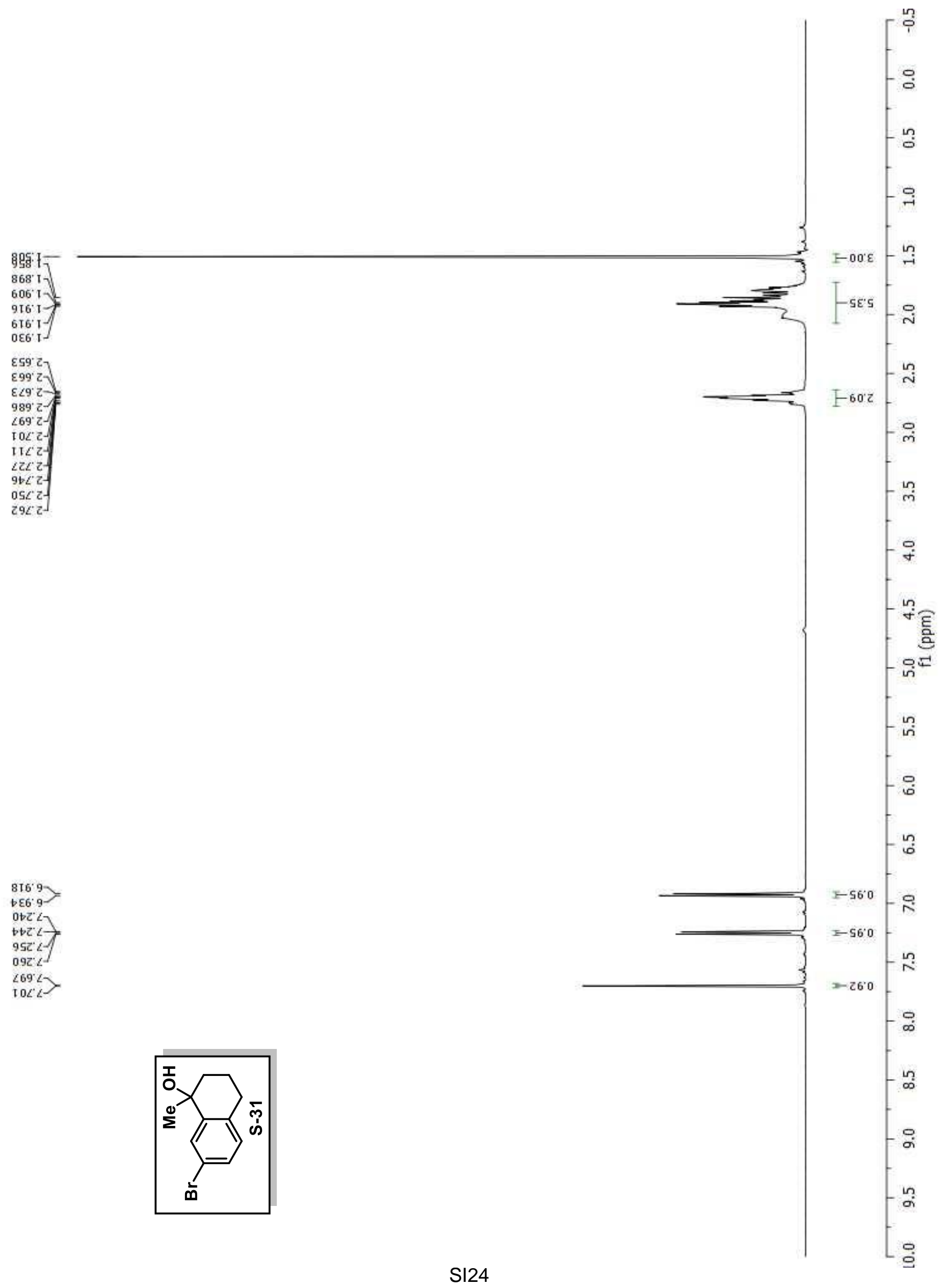




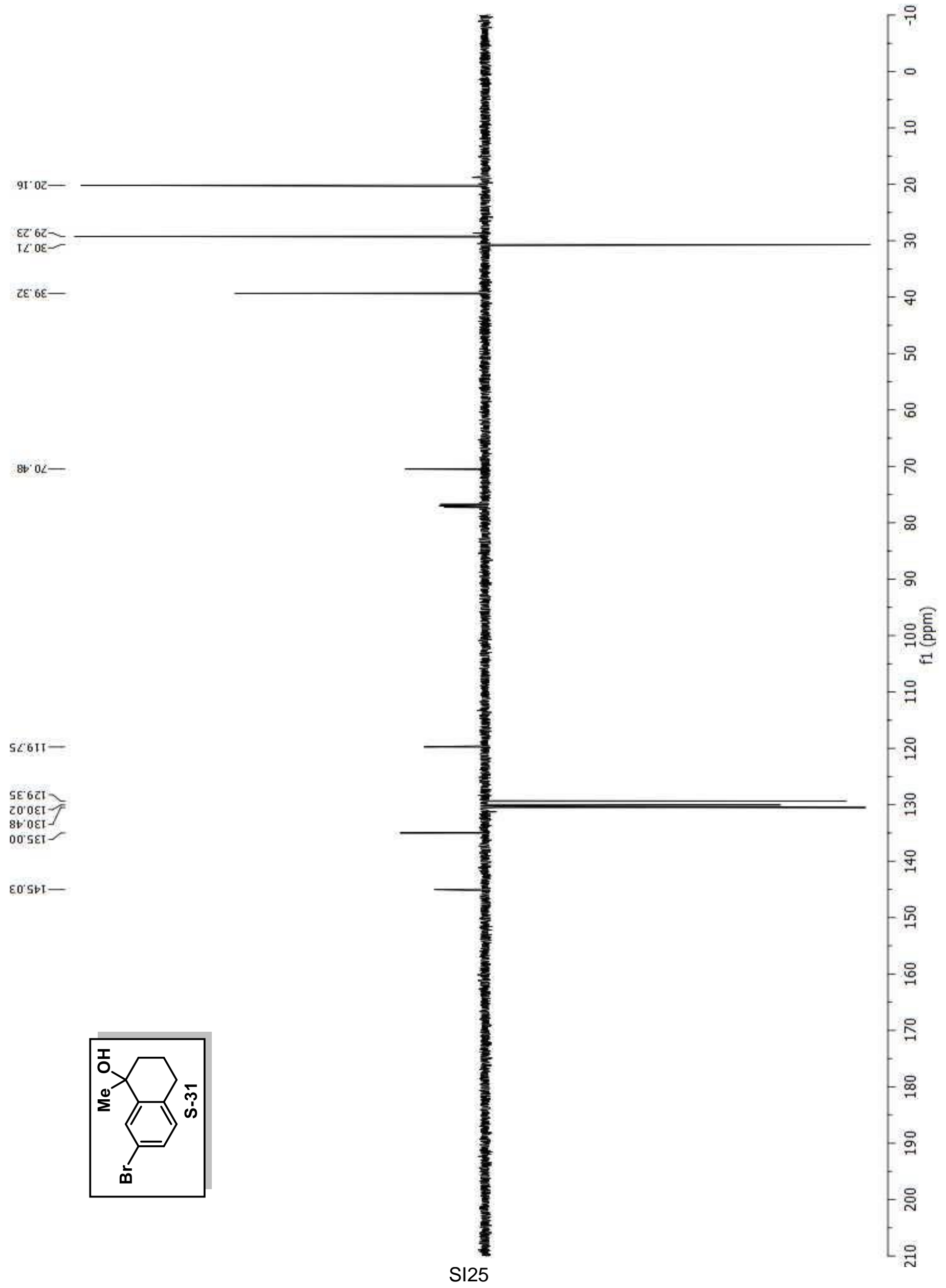




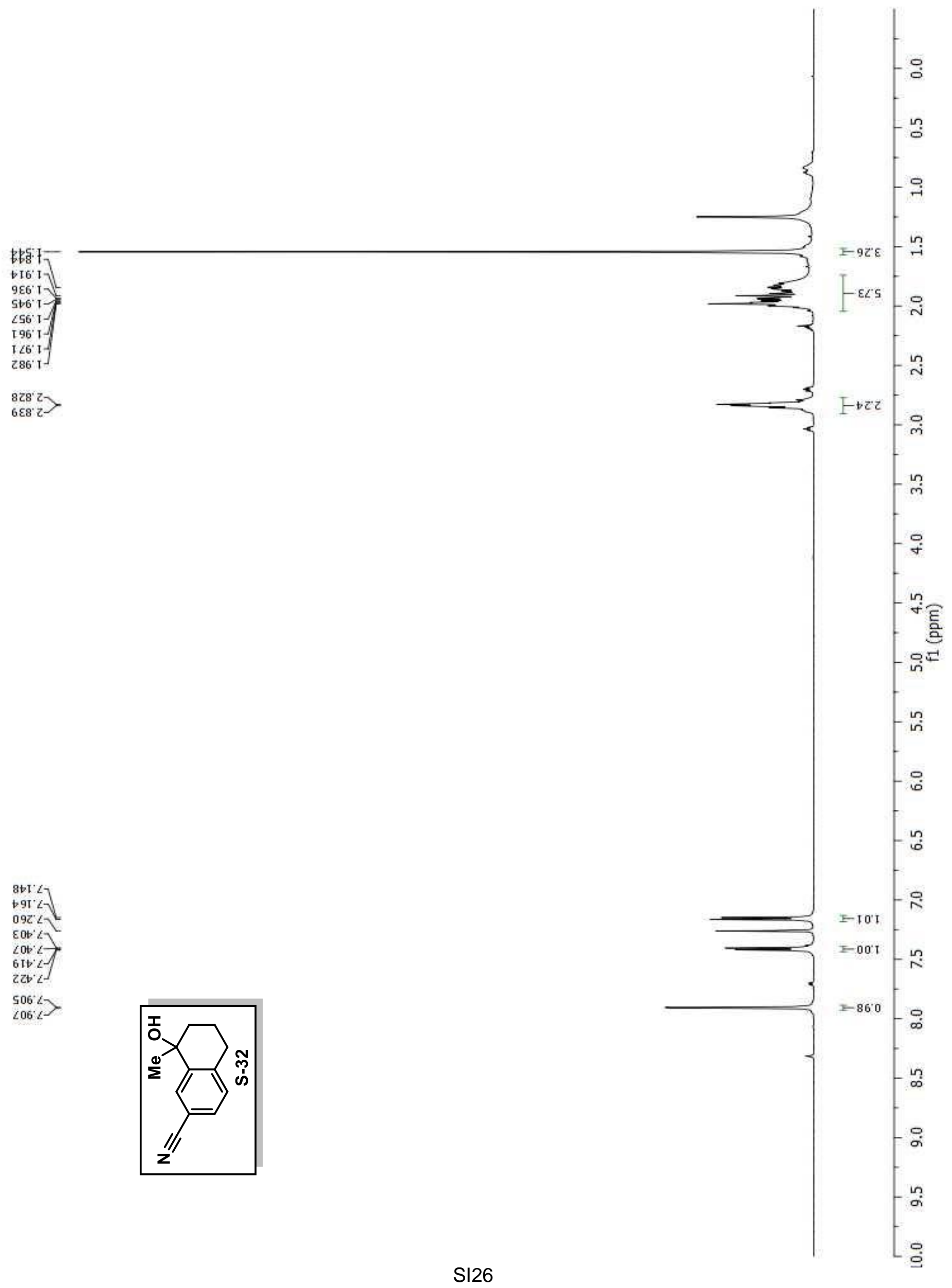




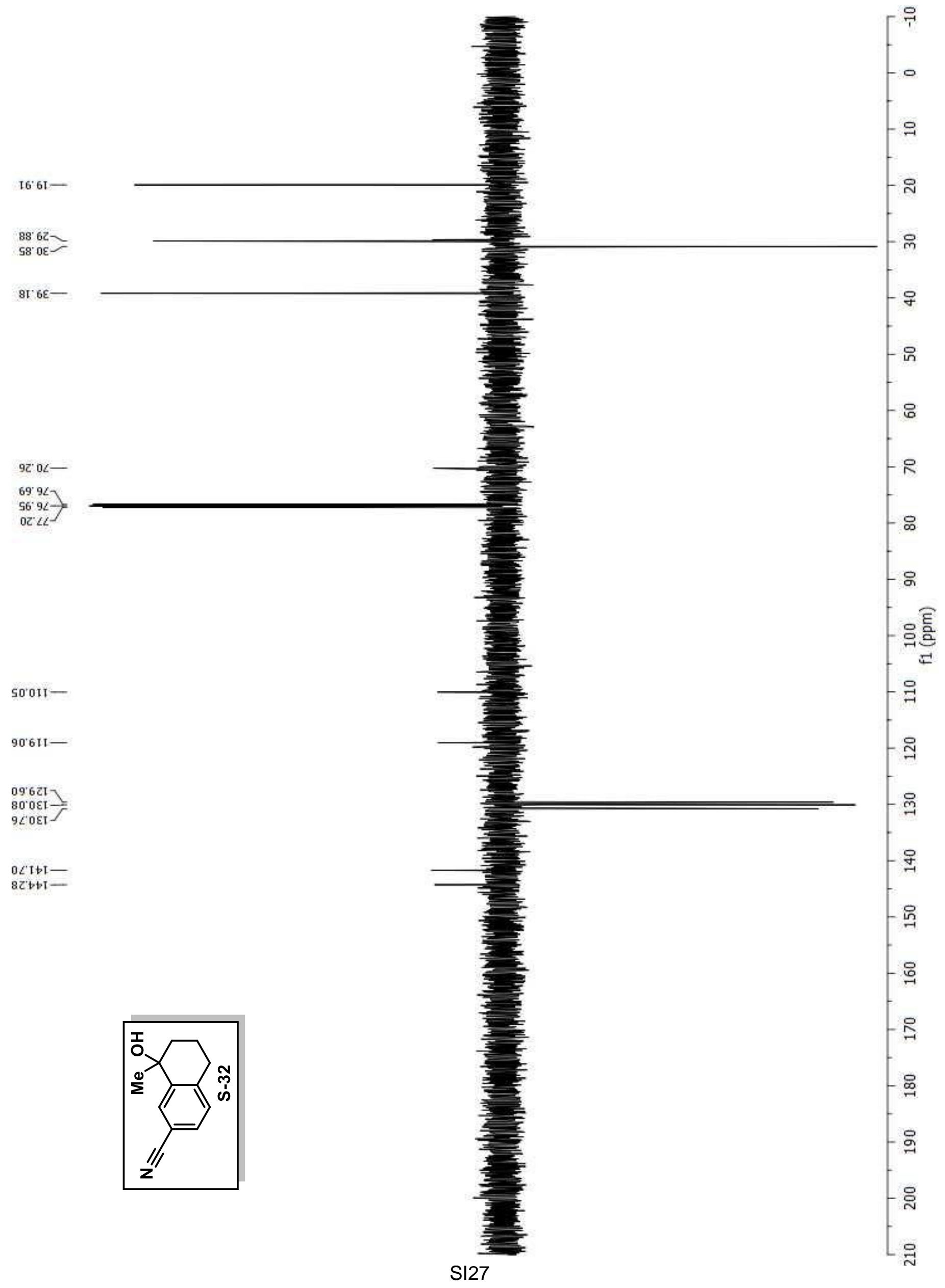




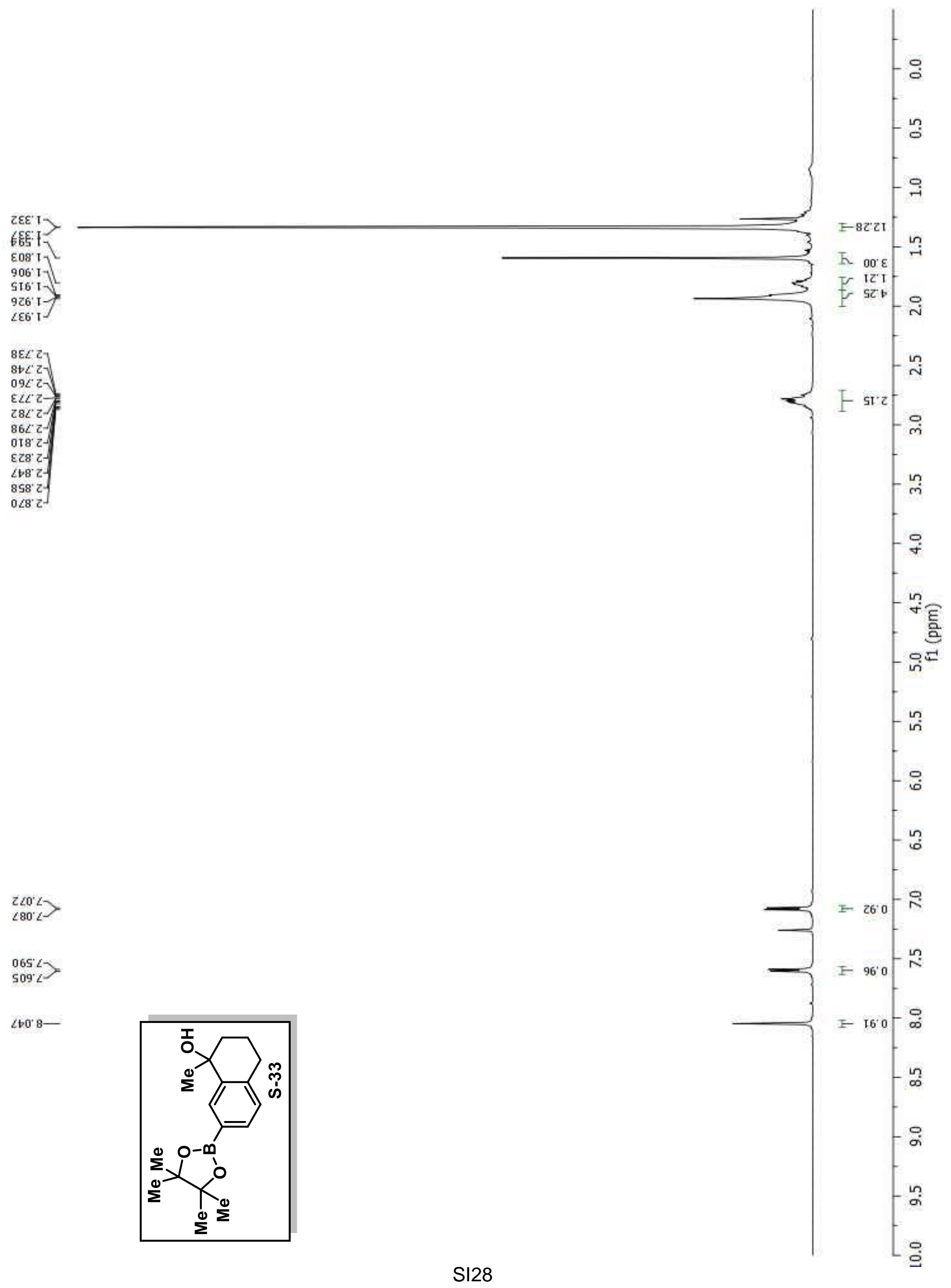




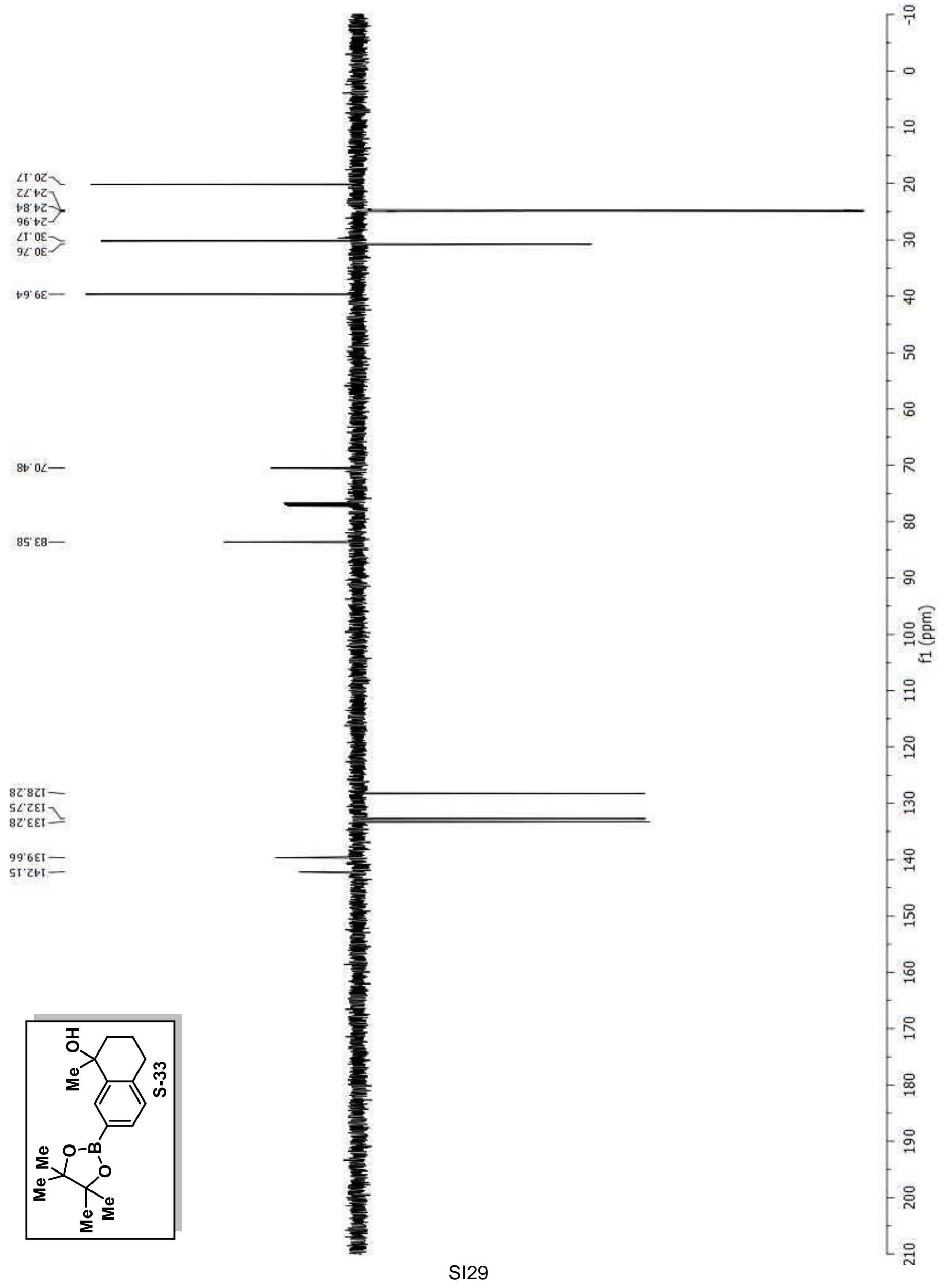




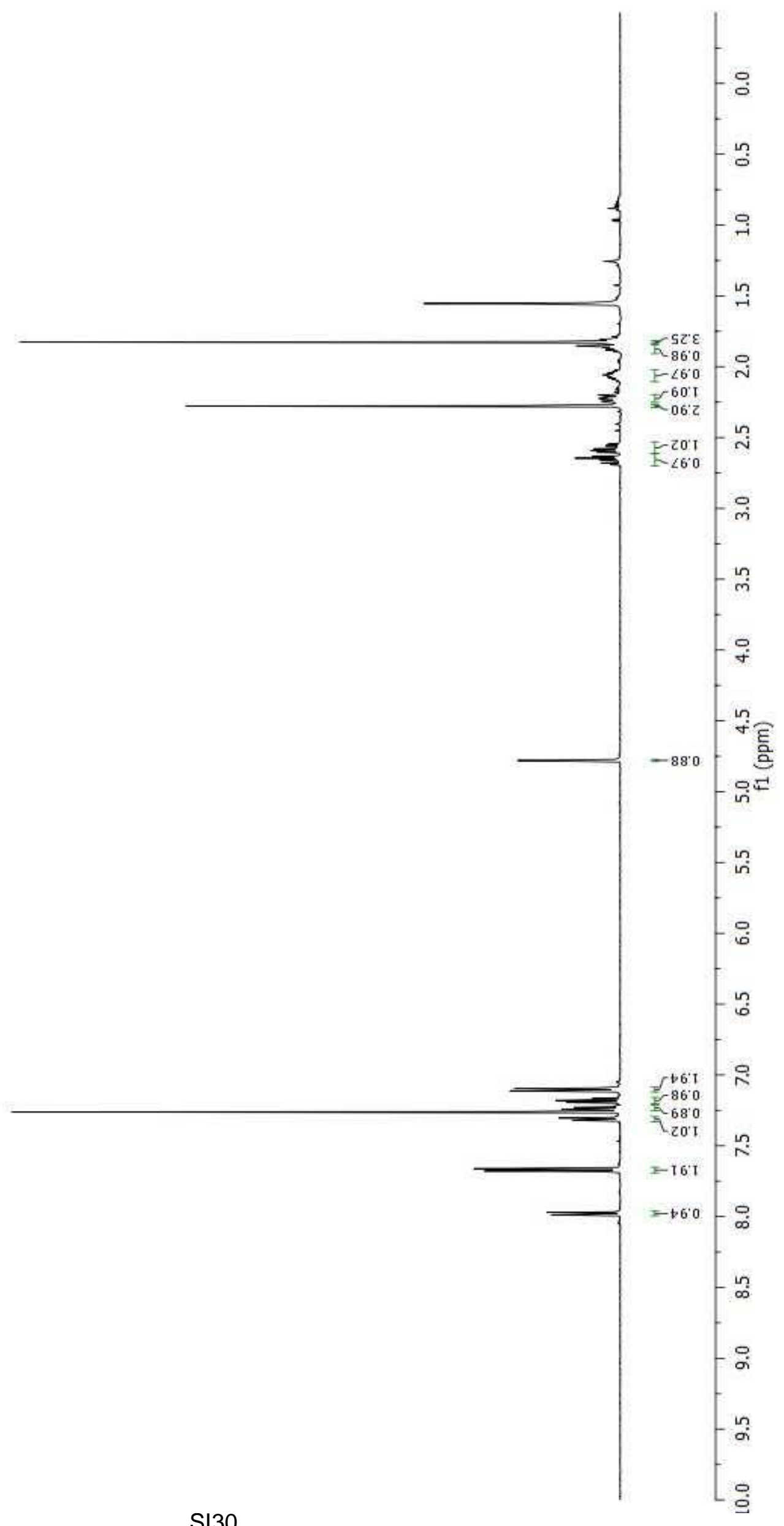




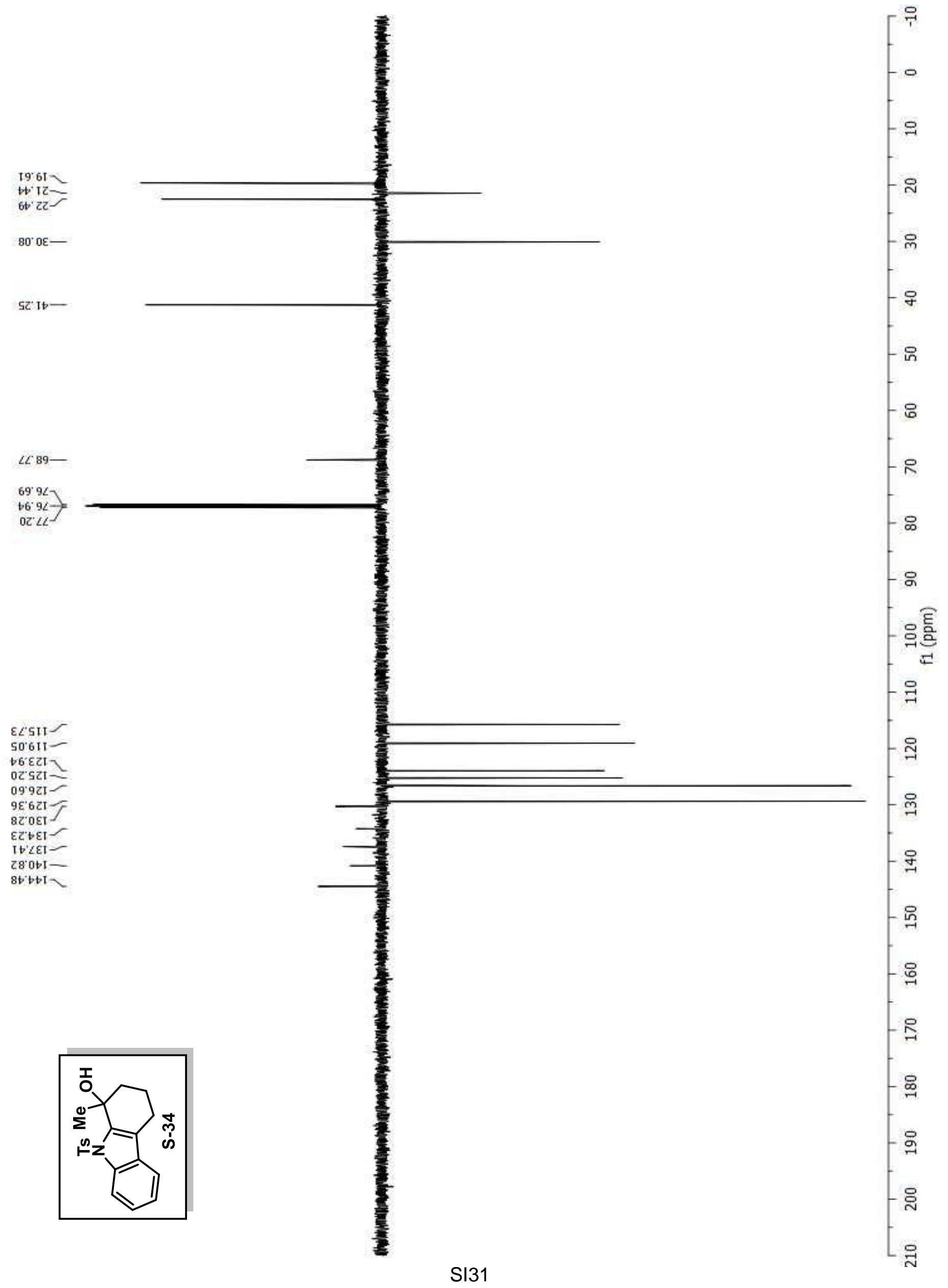




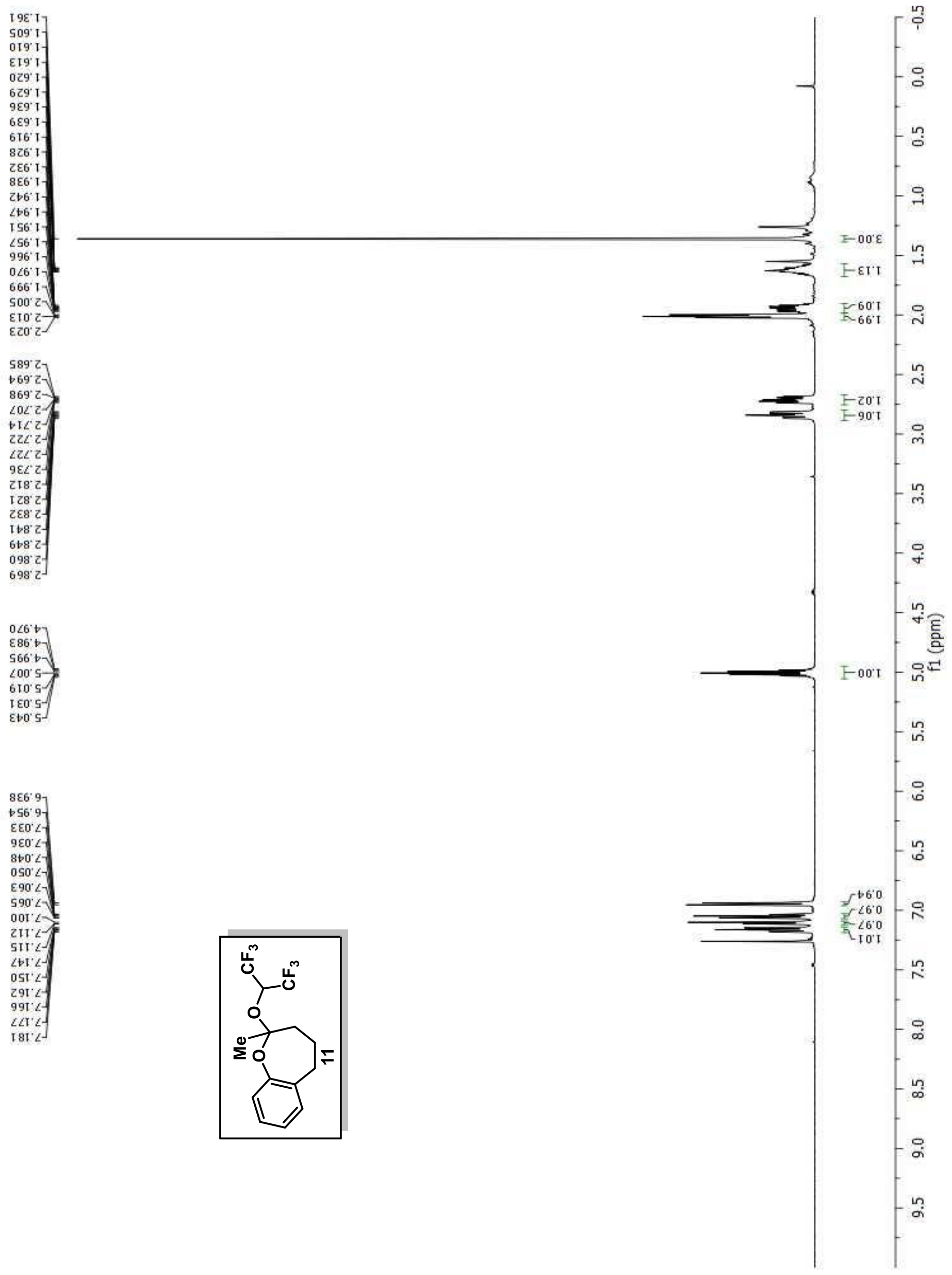




$$
\frac{1}{y}
$$




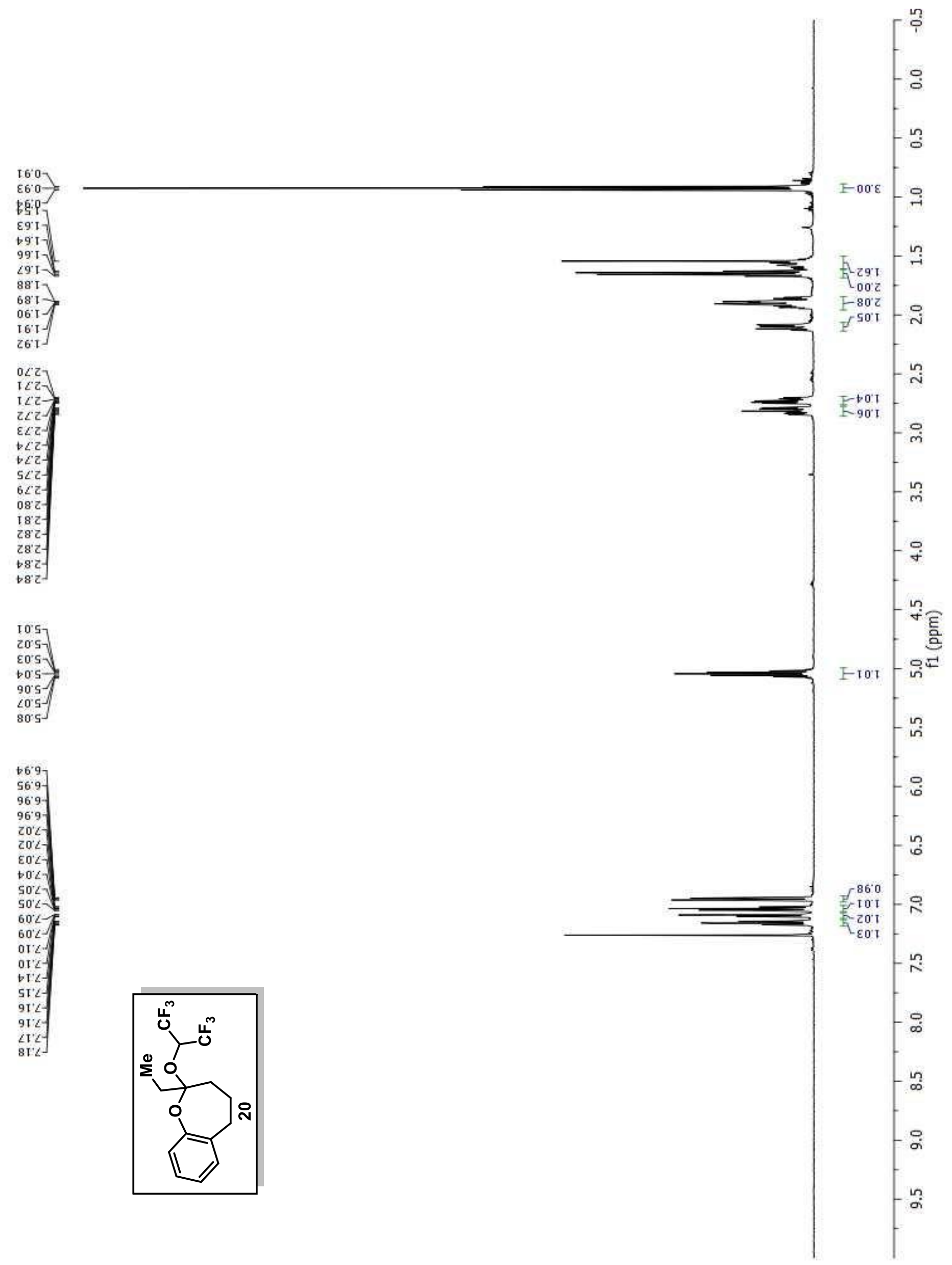




$$
\text { it }
$$




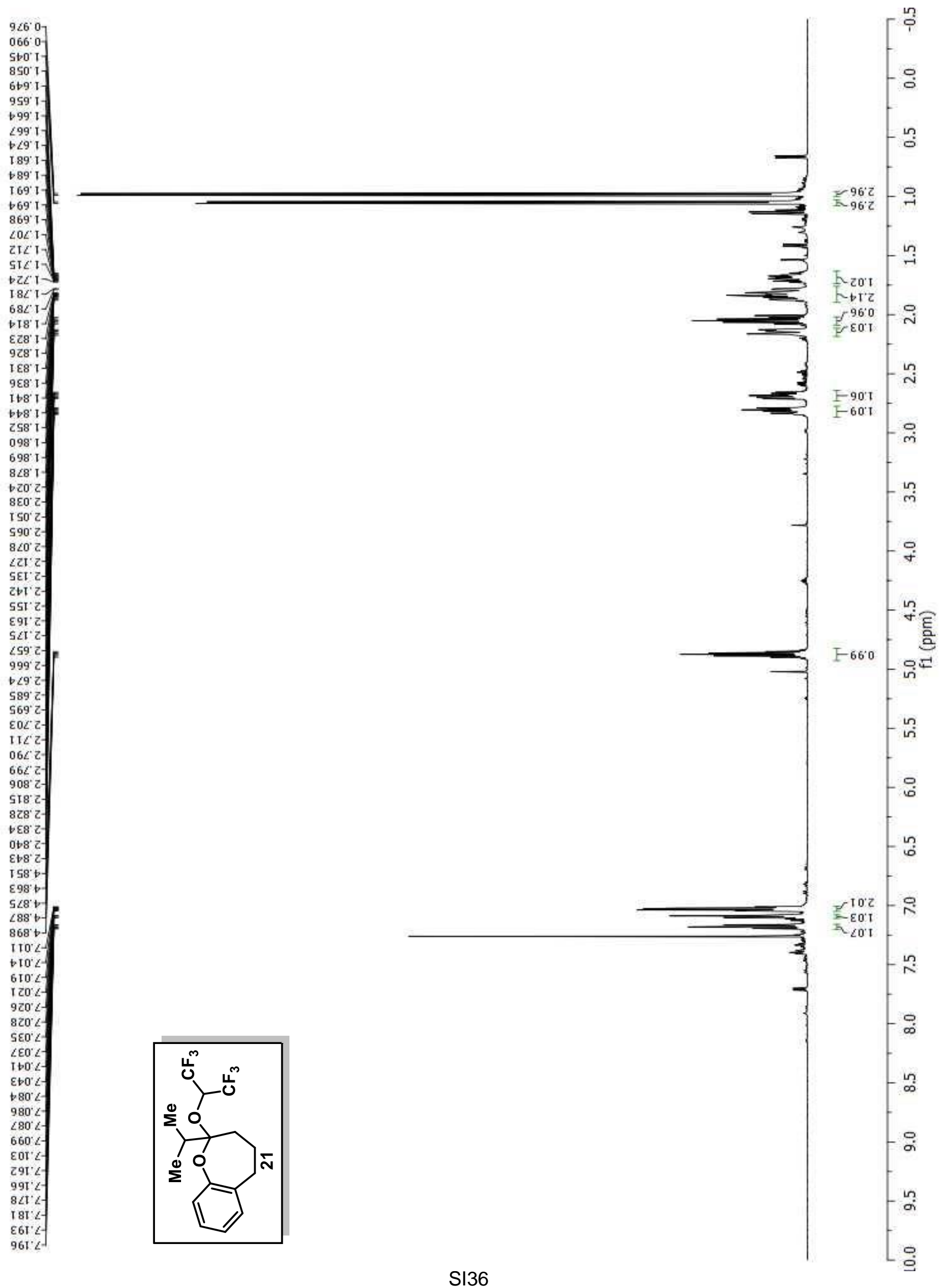




\section{(}

IZZZI-

$\mathrm{ZI} b \mathrm{ZI}-$

$\angle C C Z I-$

$60^{\circ} 08 \mathrm{I}-$

हع
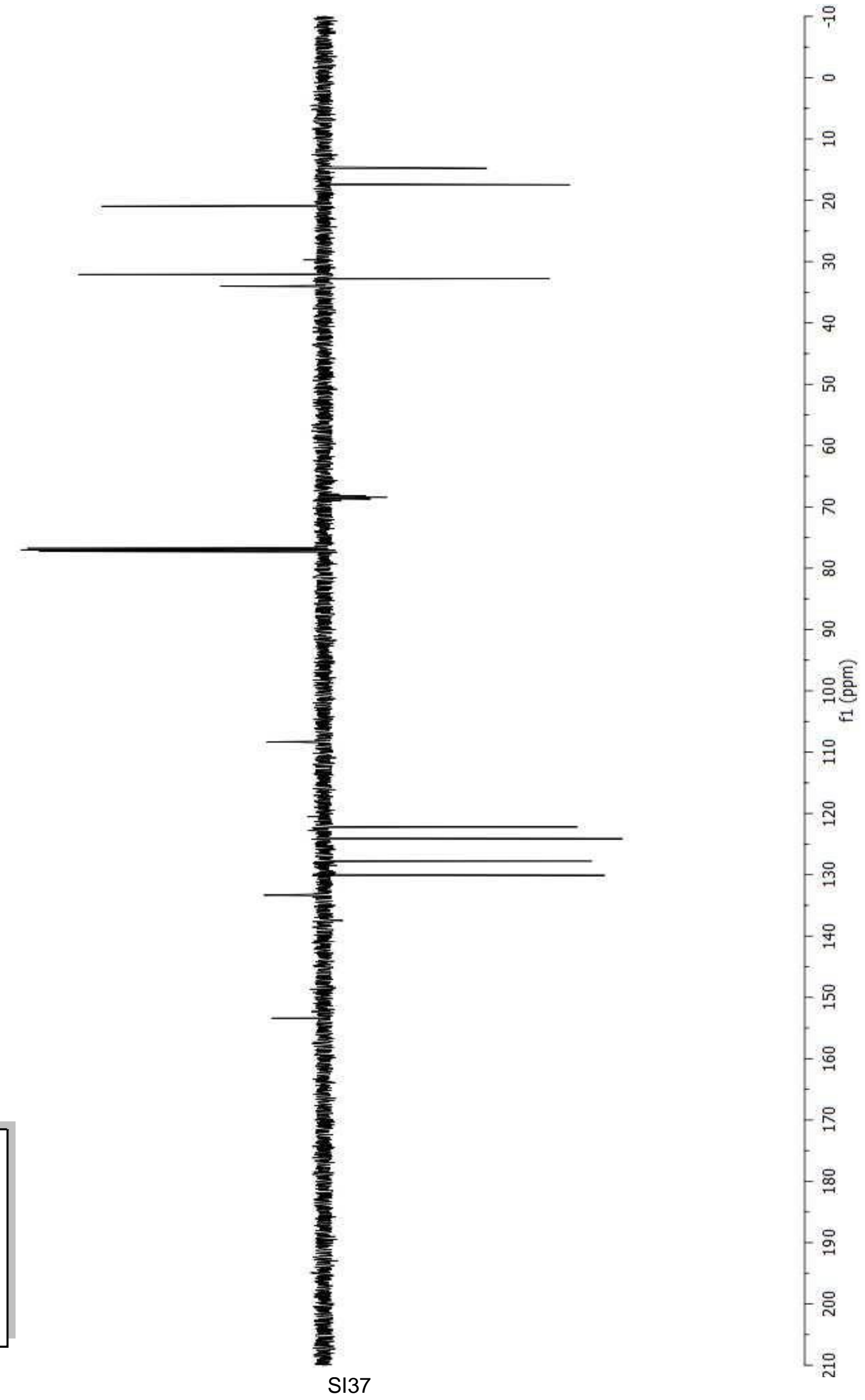
$\angle 06^{\circ} \mathrm{T}$

$016 \mathrm{~T}$

SZI' S-

LEI:S-

601 's-

$\angle 869$

$686^{\prime} 9$

$\varepsilon 00^{\circ} \angle$

$500^{\prime} \angle$

$550^{\circ}<7$

$\angle 50^{\circ} \angle$

$690^{\circ} \mathrm{L}$

$\mathrm{Z} \angle 0^{\circ} \mathrm{C}$

$+80^{\prime} \angle$

$\angle 80^{\circ} \mathrm{C}$

ZEI' $\angle$

SET $\angle$

$\angle \mathrm{I}:$

0SI' $\angle$

$9 I^{\prime}<$

$\angle 9 T^{\prime} \angle$

$6 \angle \mathrm{I} \angle$

$281: \angle$

$+6 \mathrm{~T}^{\prime} \mathrm{C}$

$861^{\circ} \mathrm{L}$

$91 Z^{\prime} \angle$

I $22:$

$822 \angle$

$\downarrow \varepsilon Z^{\prime} \angle-$

$8 \varepsilon C^{\prime}<$

$b t Z^{\prime} \angle$

$\varepsilon S Z$ :

$092: \angle-$

$\angle 9 C^{\prime} \angle$

$692 \mathrm{~L}$
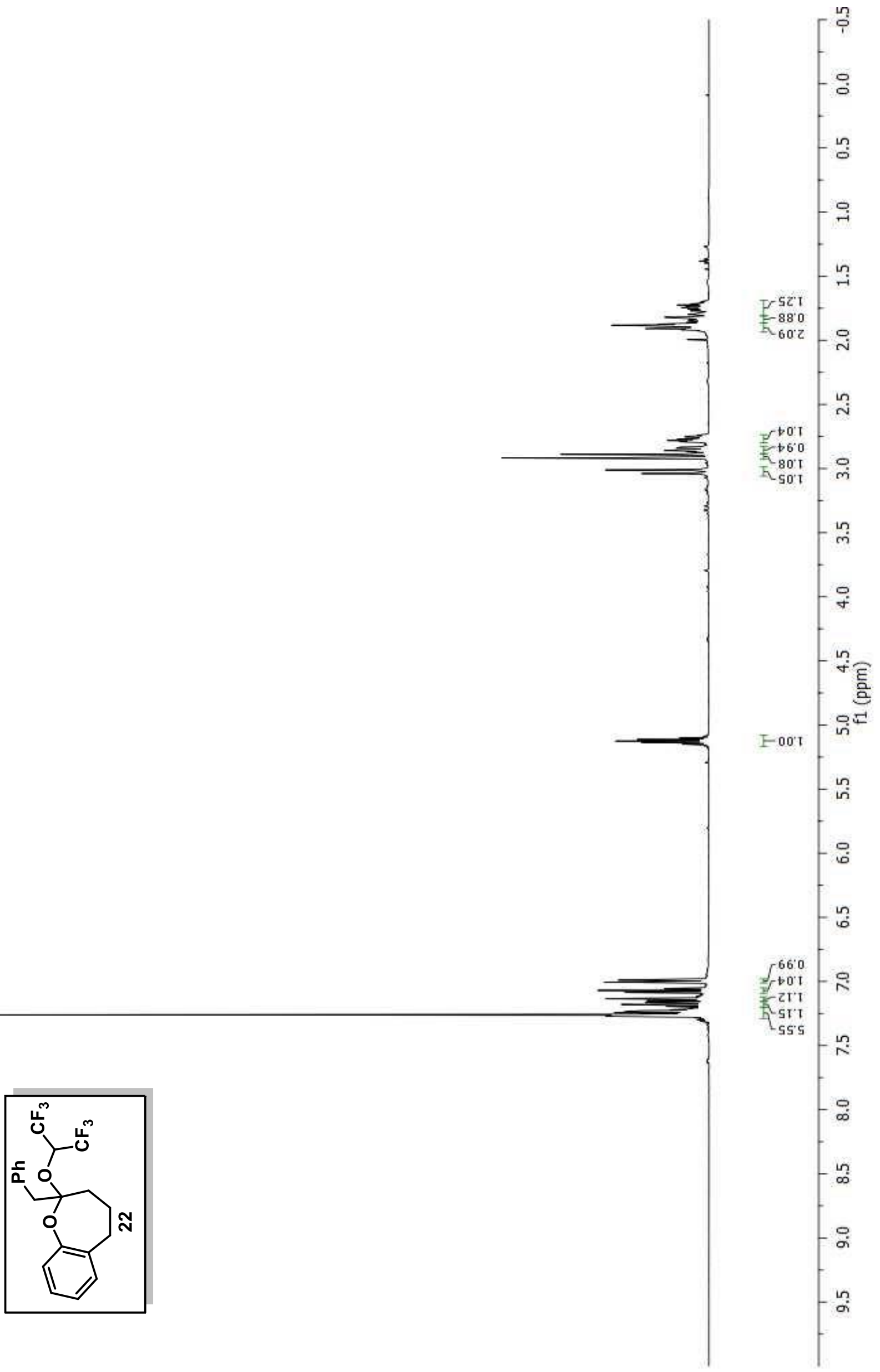
E9: $12-$

$$
\text { 69. } 1 \varepsilon-
$$$$
\text { } 0 \varepsilon \text { } \varsigma \varepsilon-
$$$$
\text { 9I ह ह - }
$$$$
\text { 0I' } 901-
$$

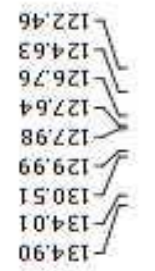

$06251-$

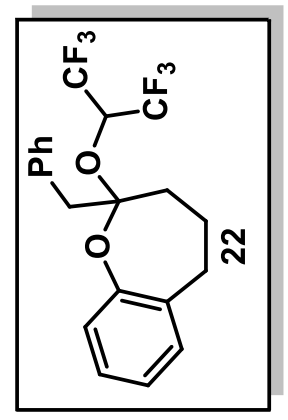

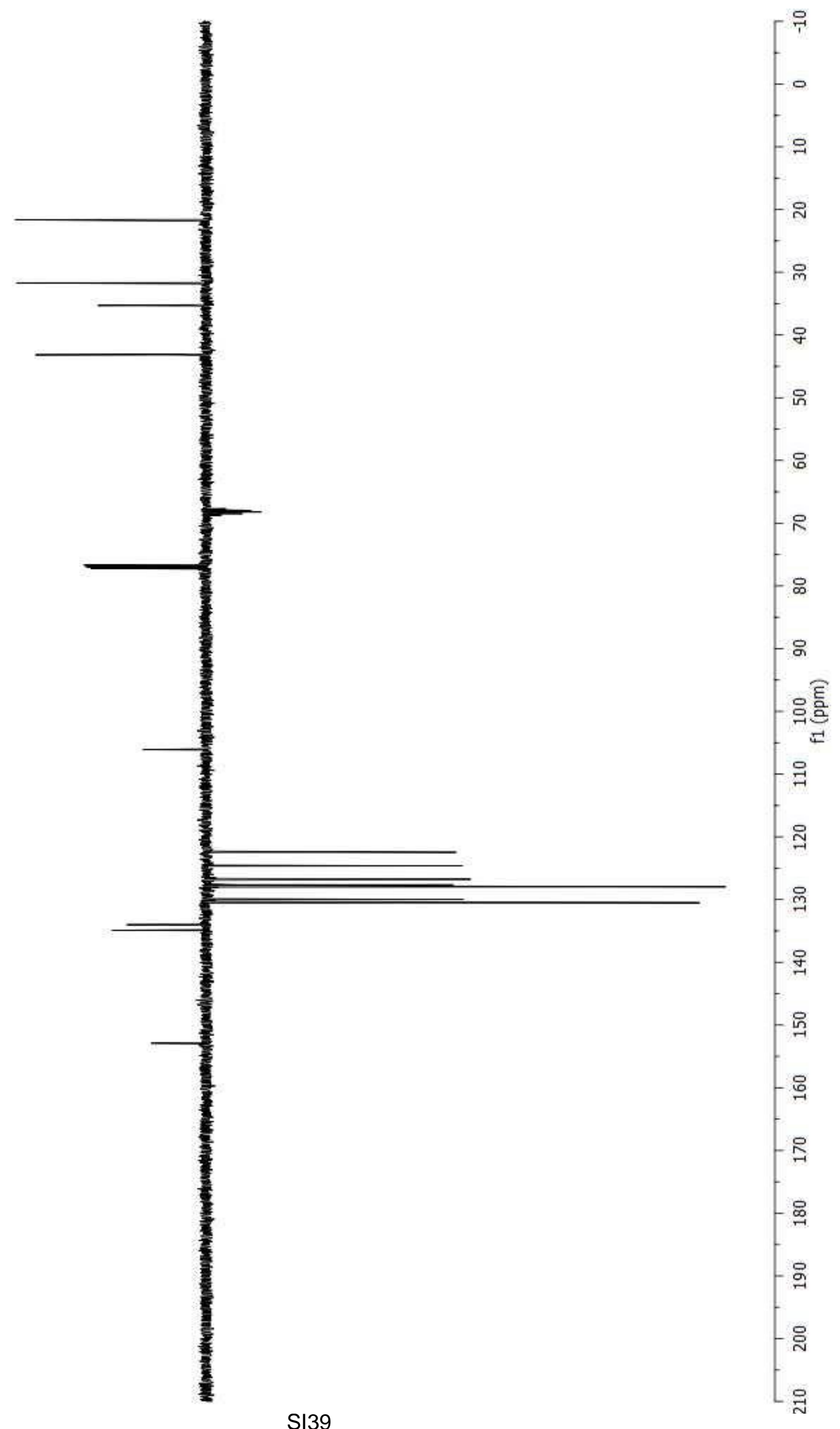




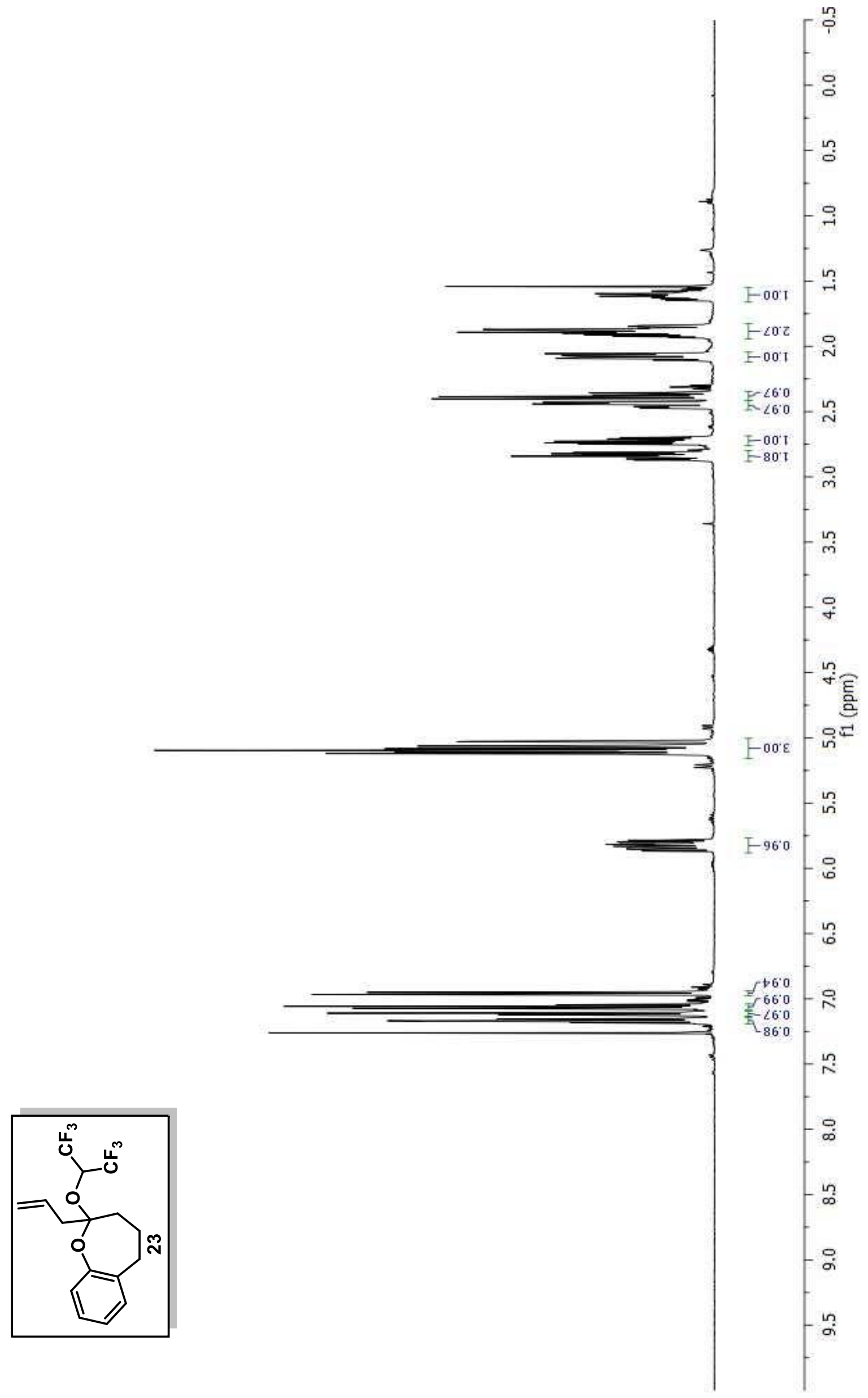




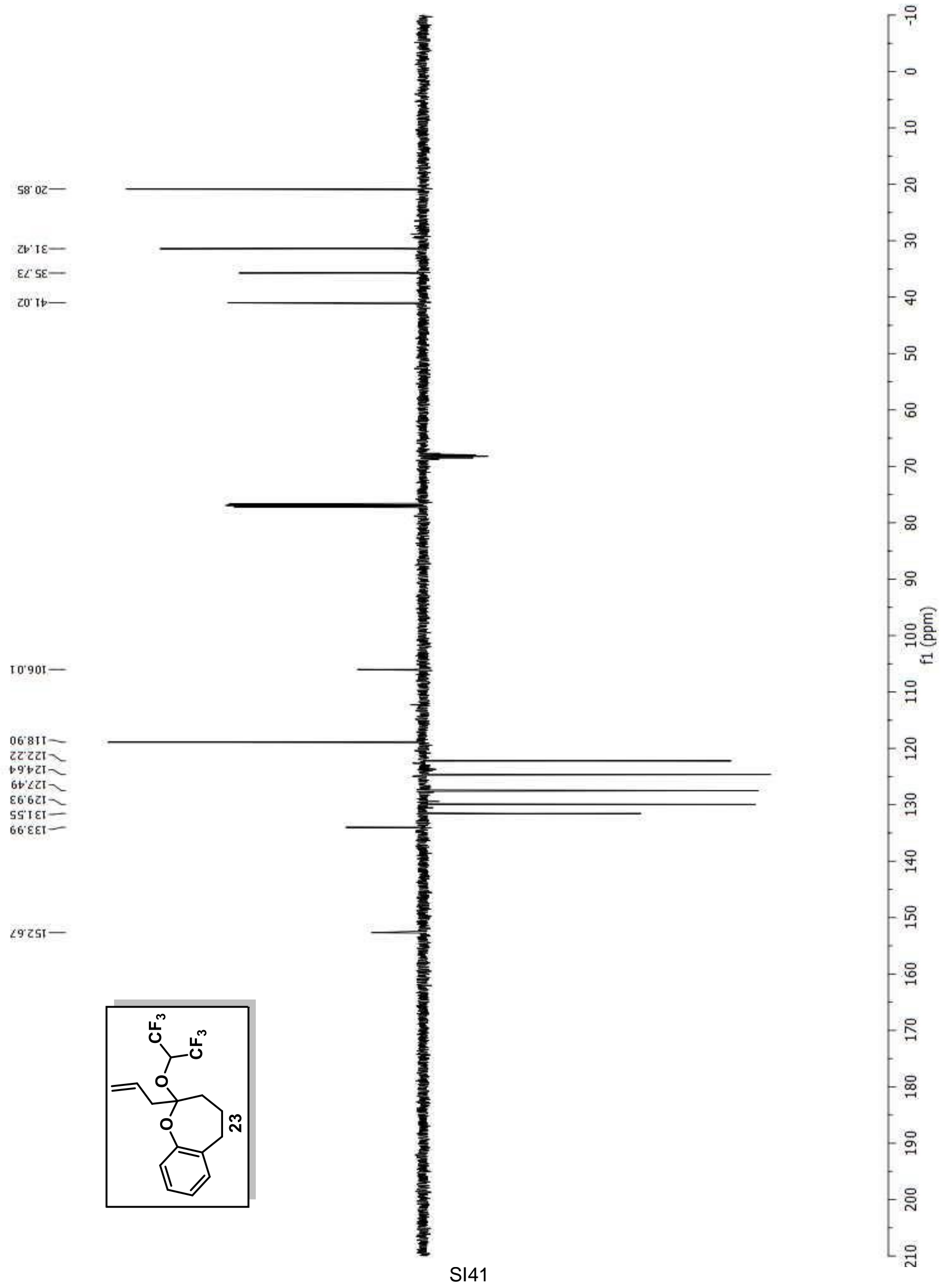




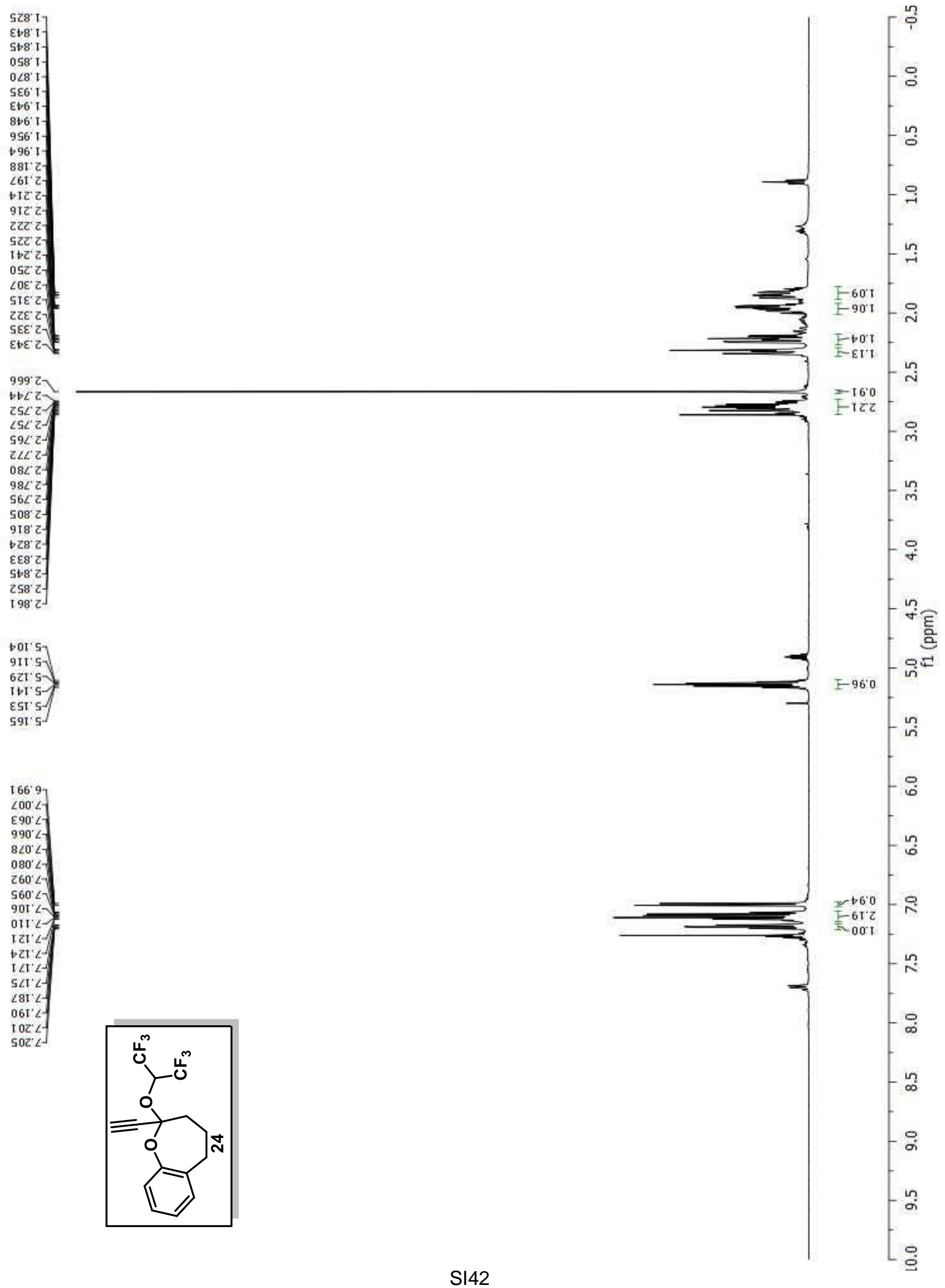



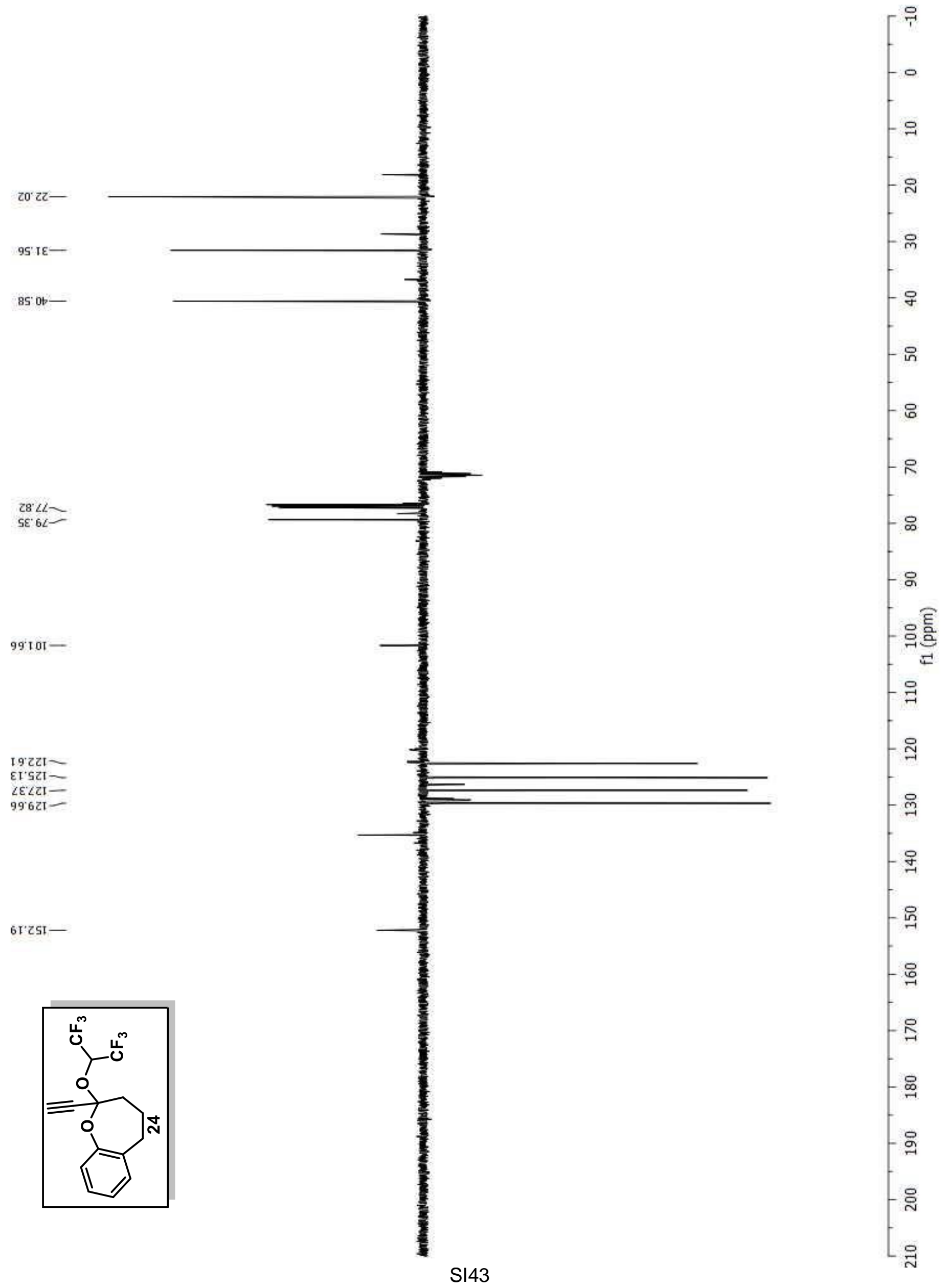


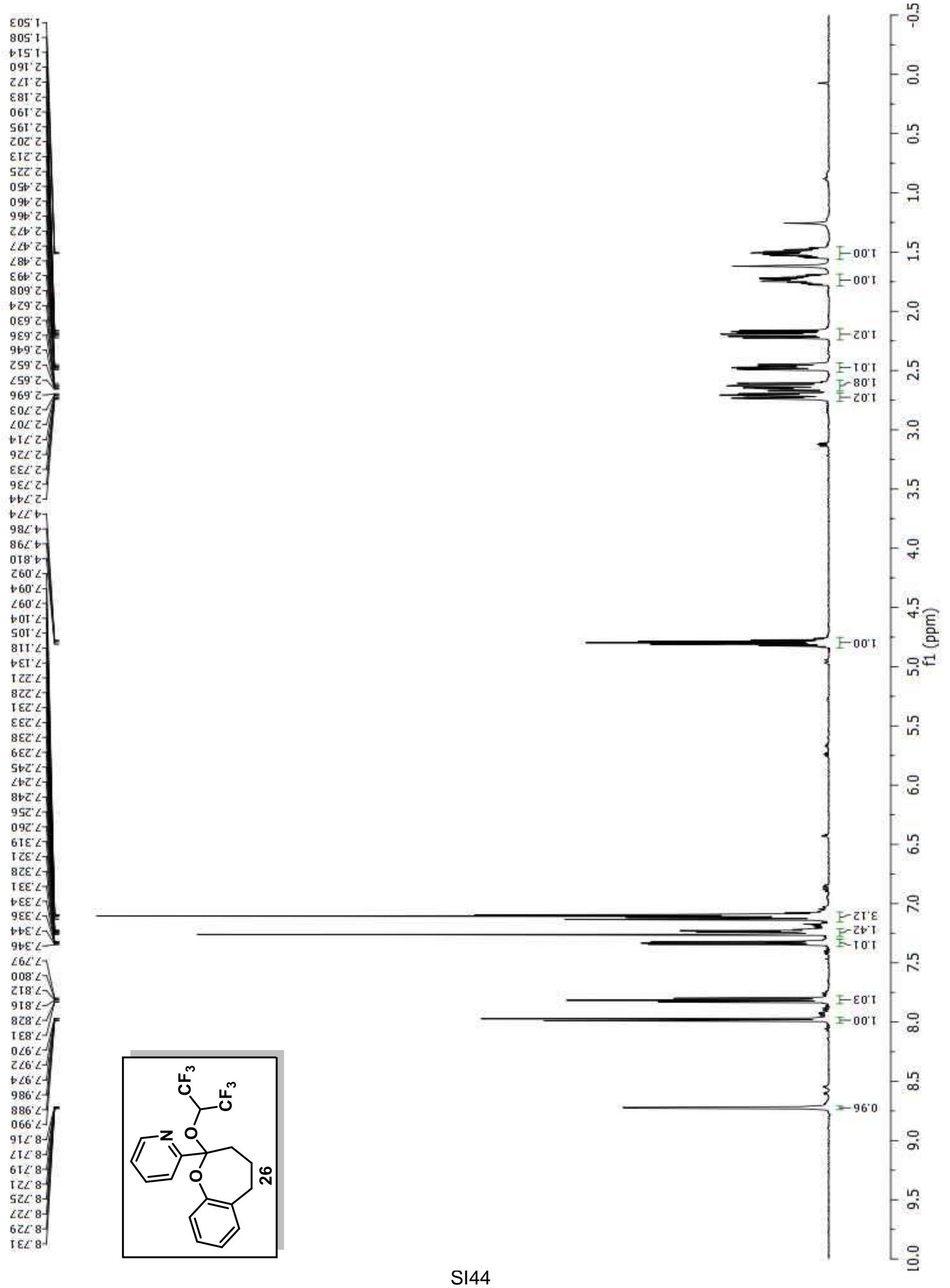




$$
\text { it }
$$




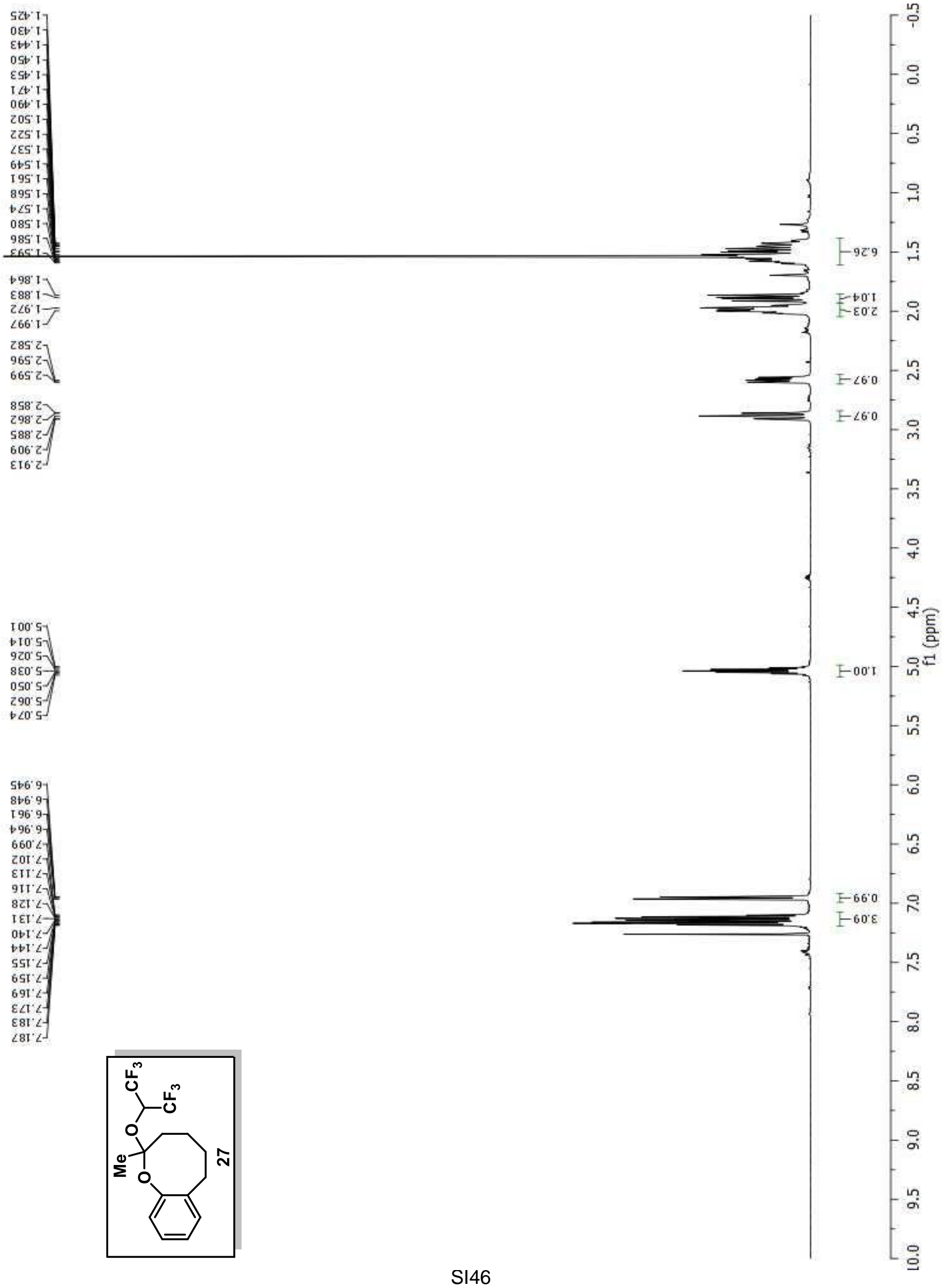




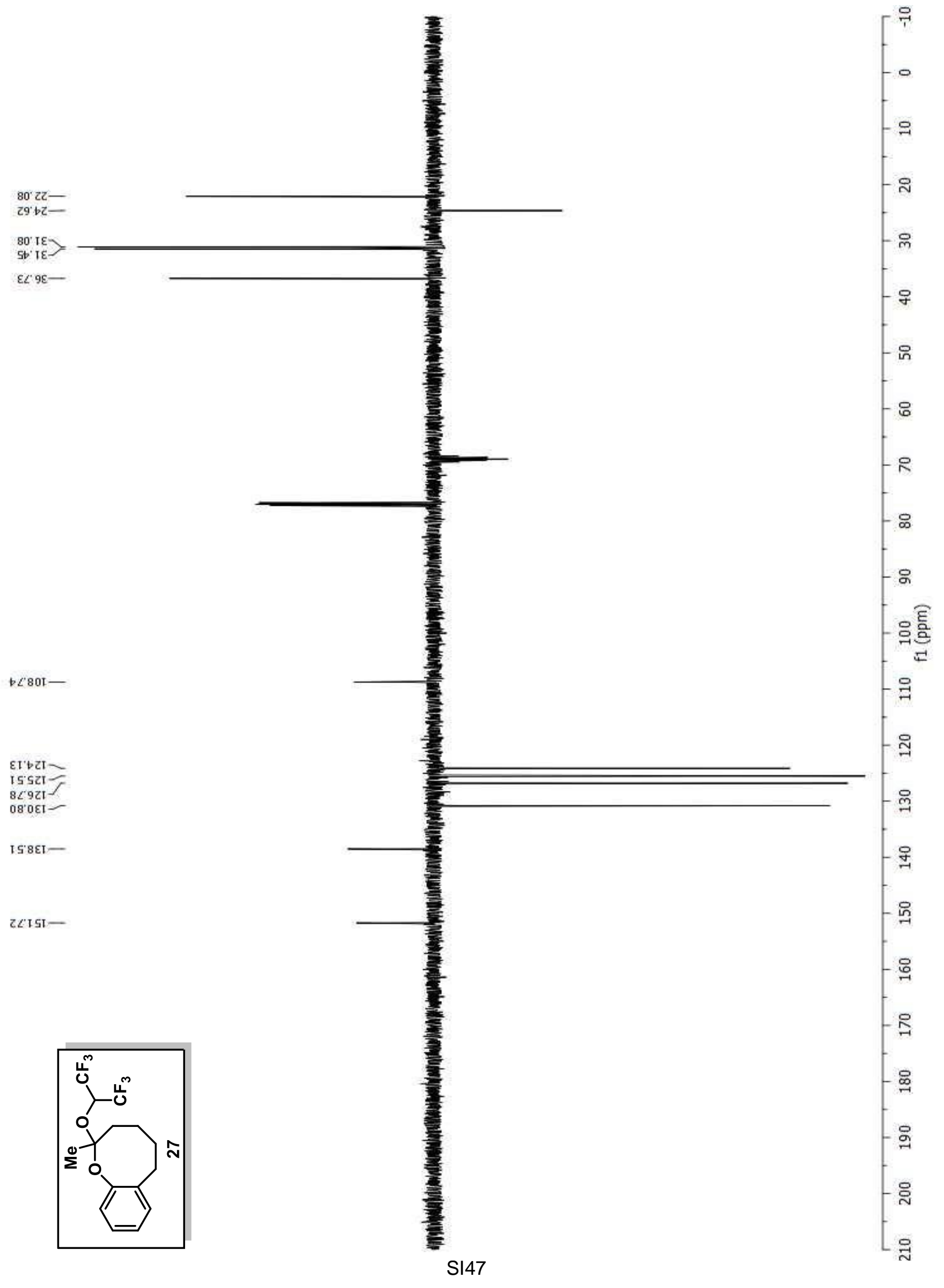




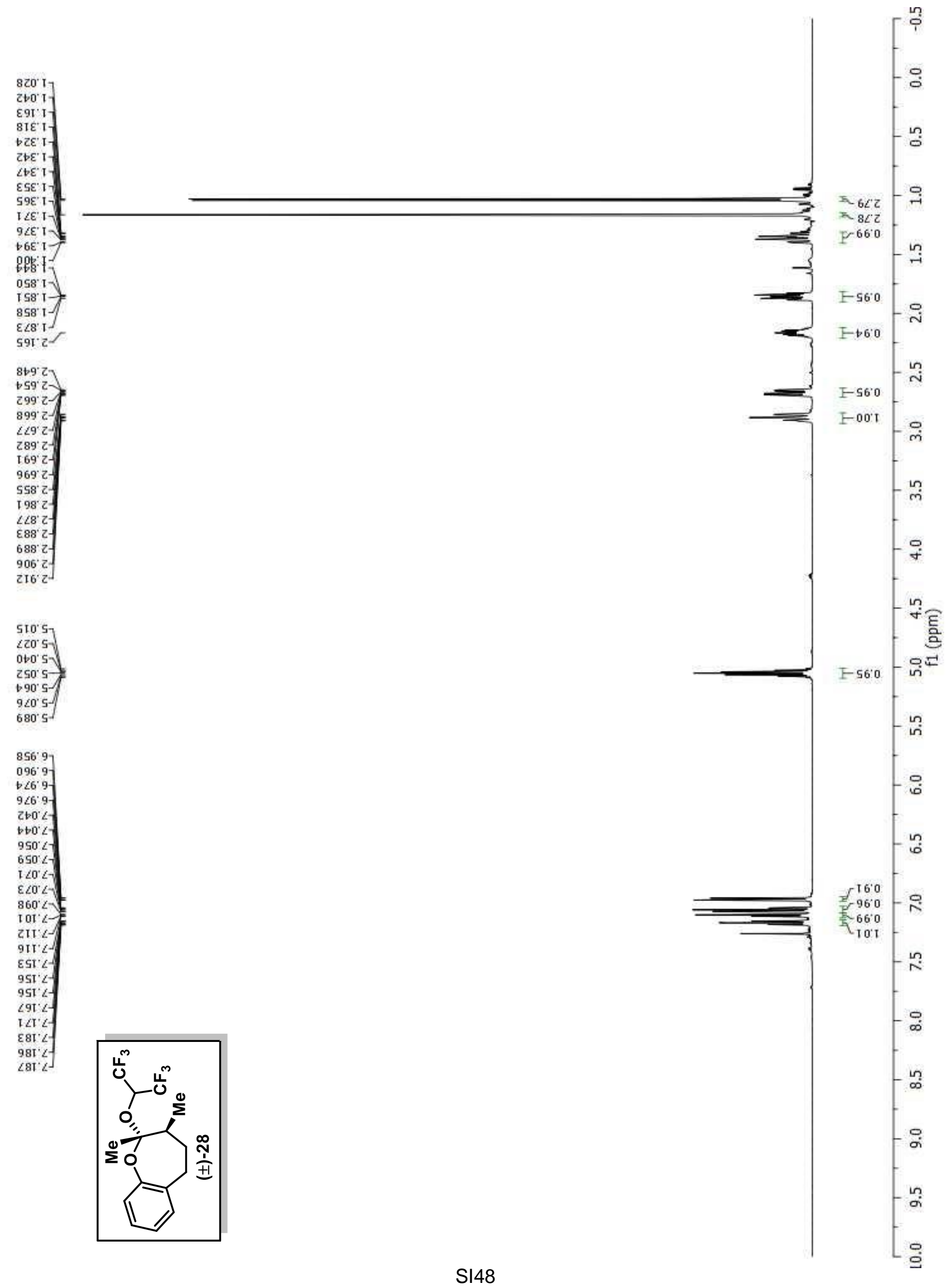




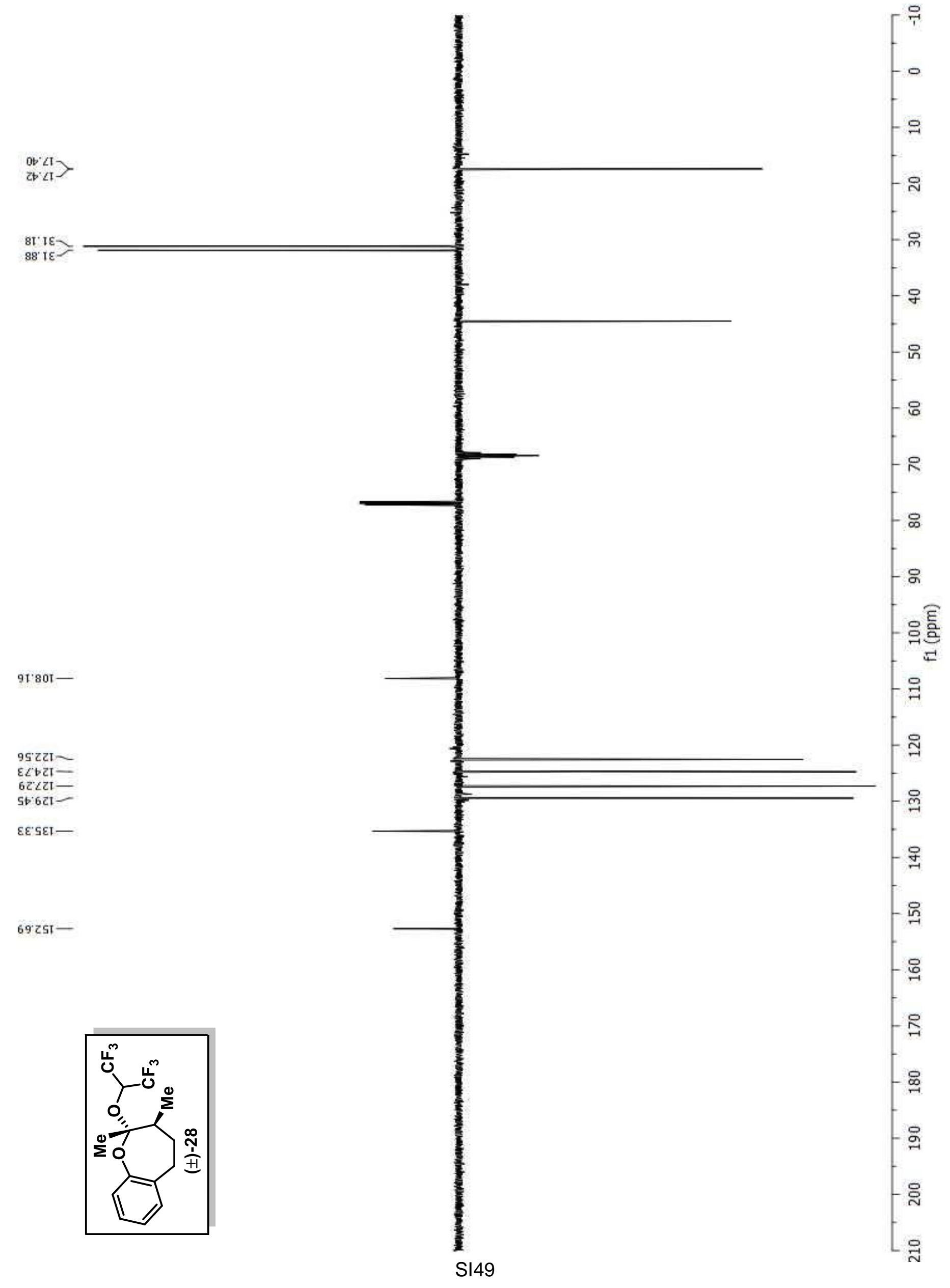




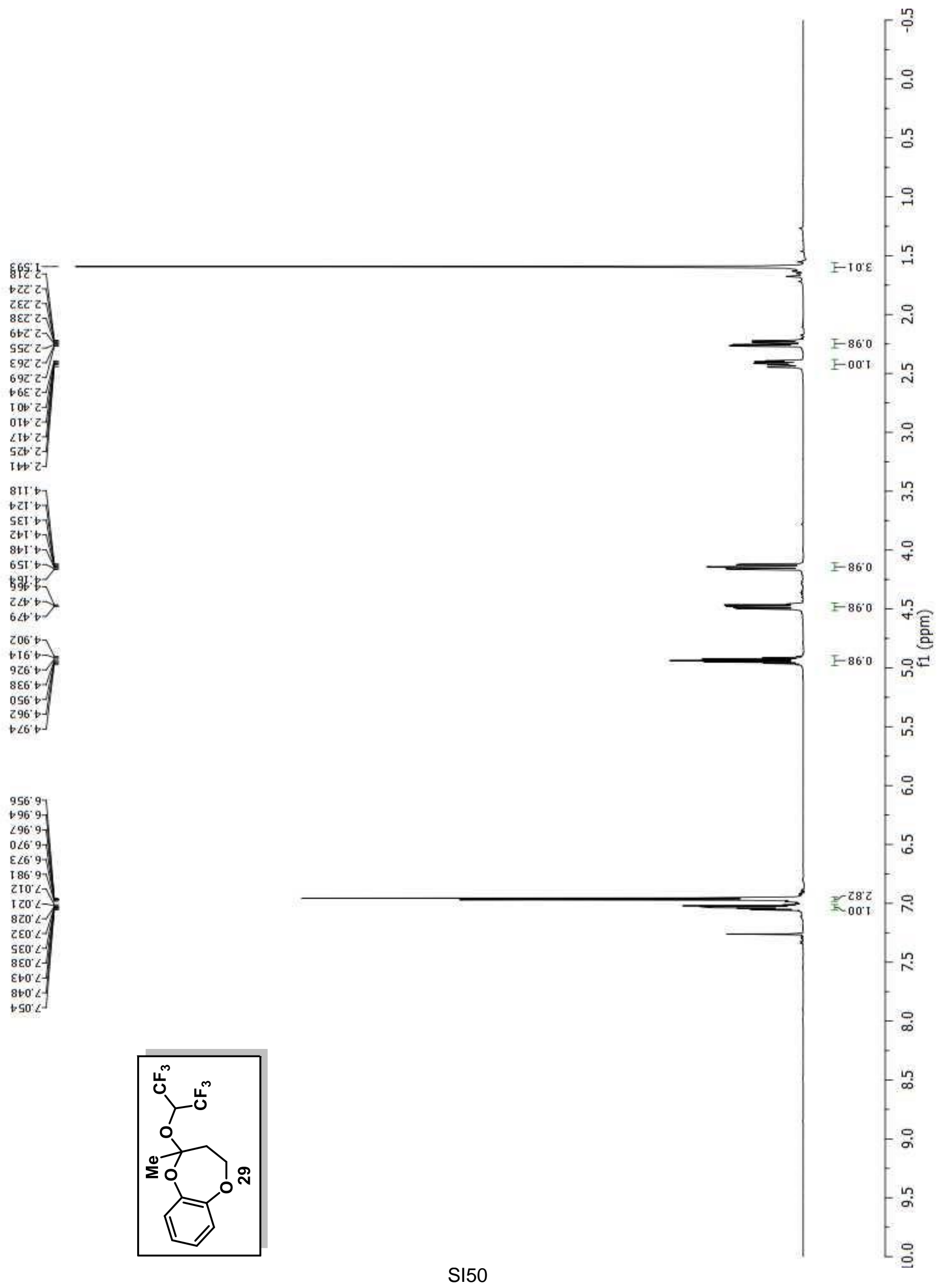




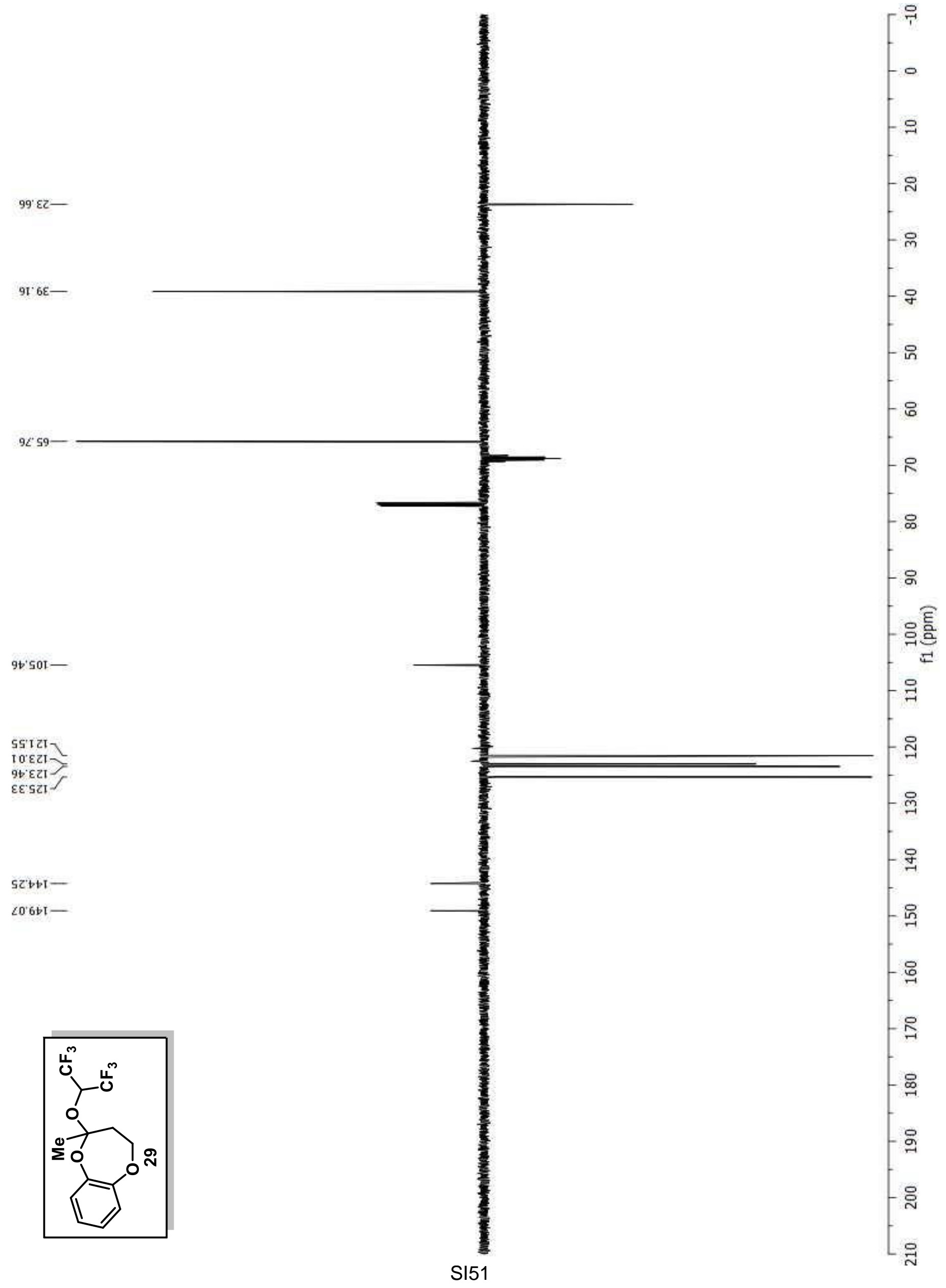


$\varepsilon \measuredangle L \varepsilon-$

$856: b$

$0 \angle 6^{\circ} b$

$\varepsilon 86^{\circ} \mathrm{b}$

566.

$\angle 00^{\circ} \mathrm{S}-$

$610^{\prime} \mathrm{s}-$

I 80 '

$\angle \circ 9 \cdot 9$

ES9:9-

659 9

699.9-7.

$5 \angle 9^{\circ} 9^{-}$

ZS8 9-

ES8: 9-

698.9-

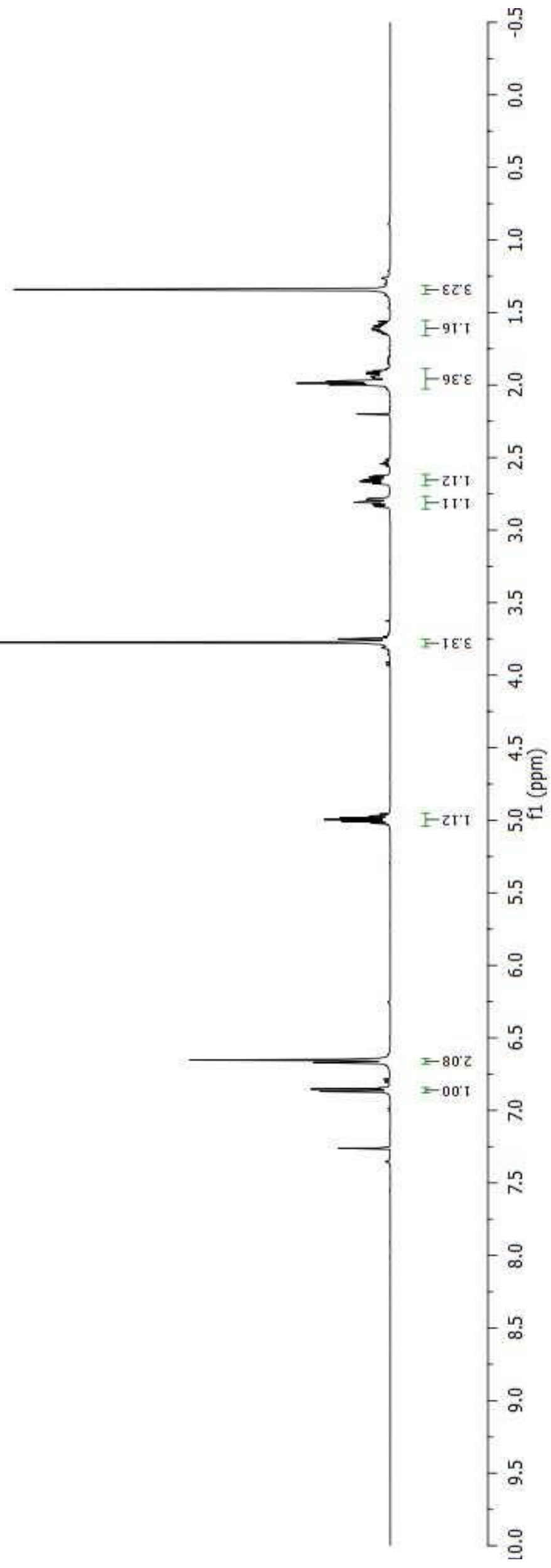




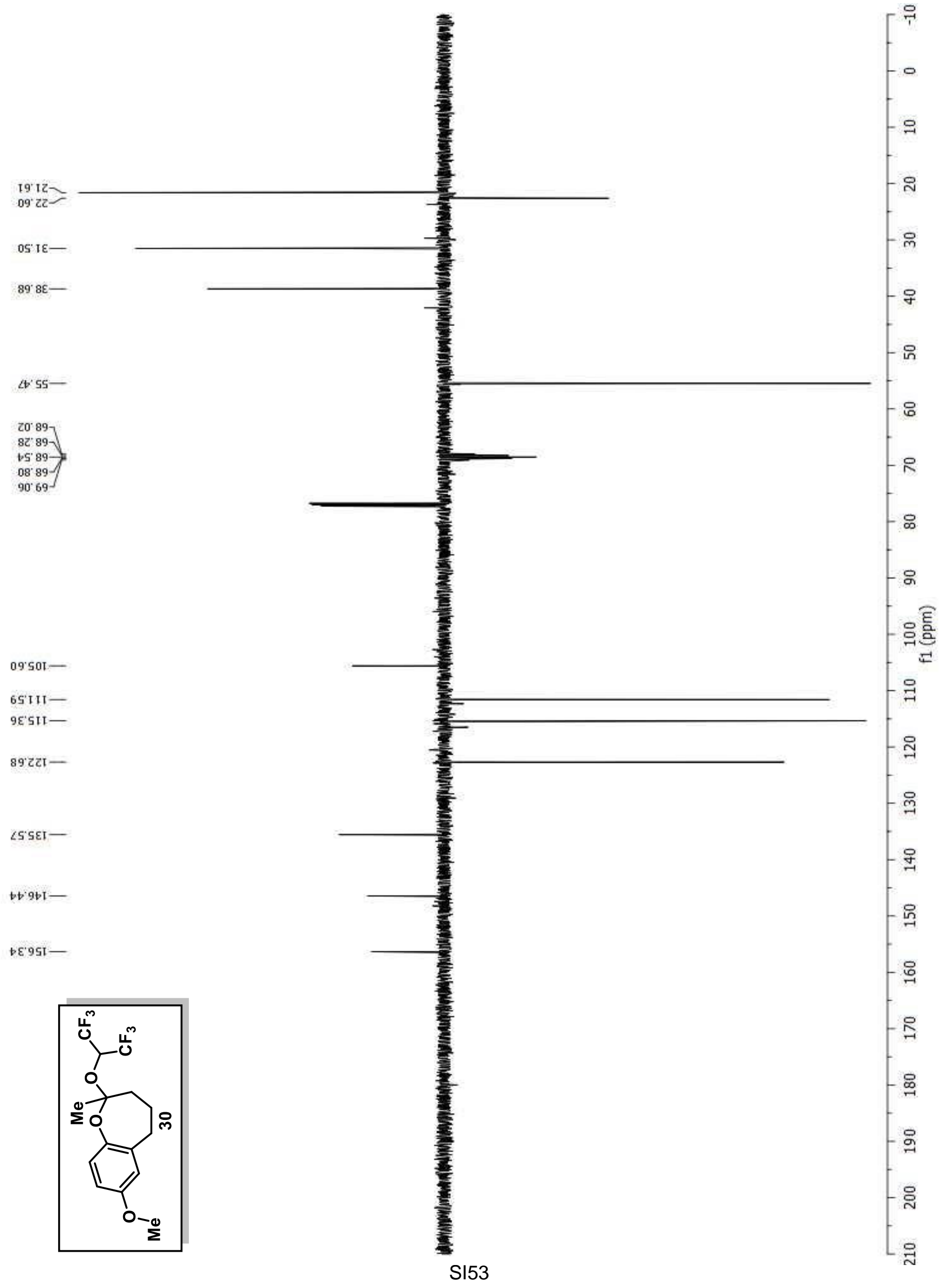




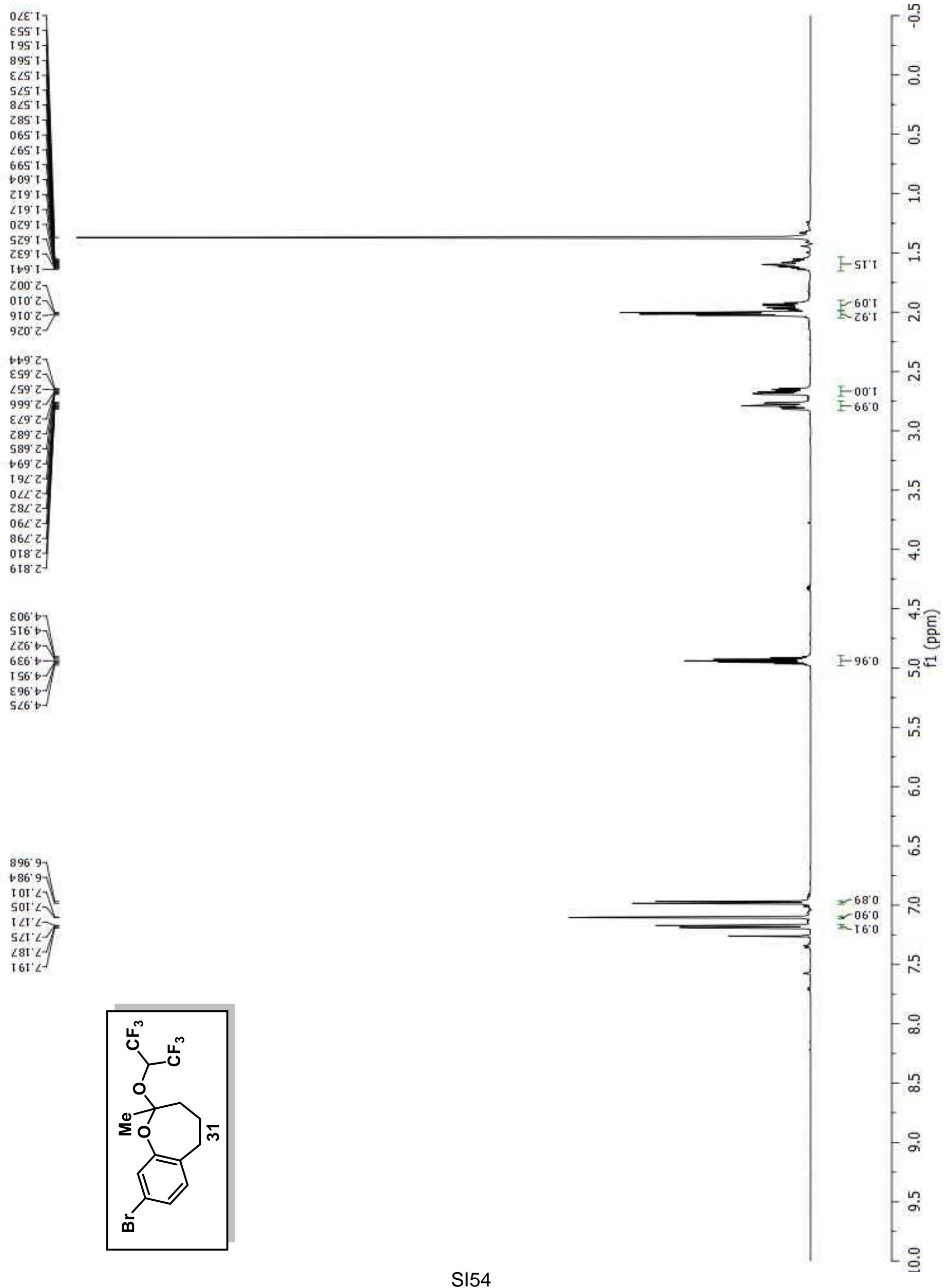




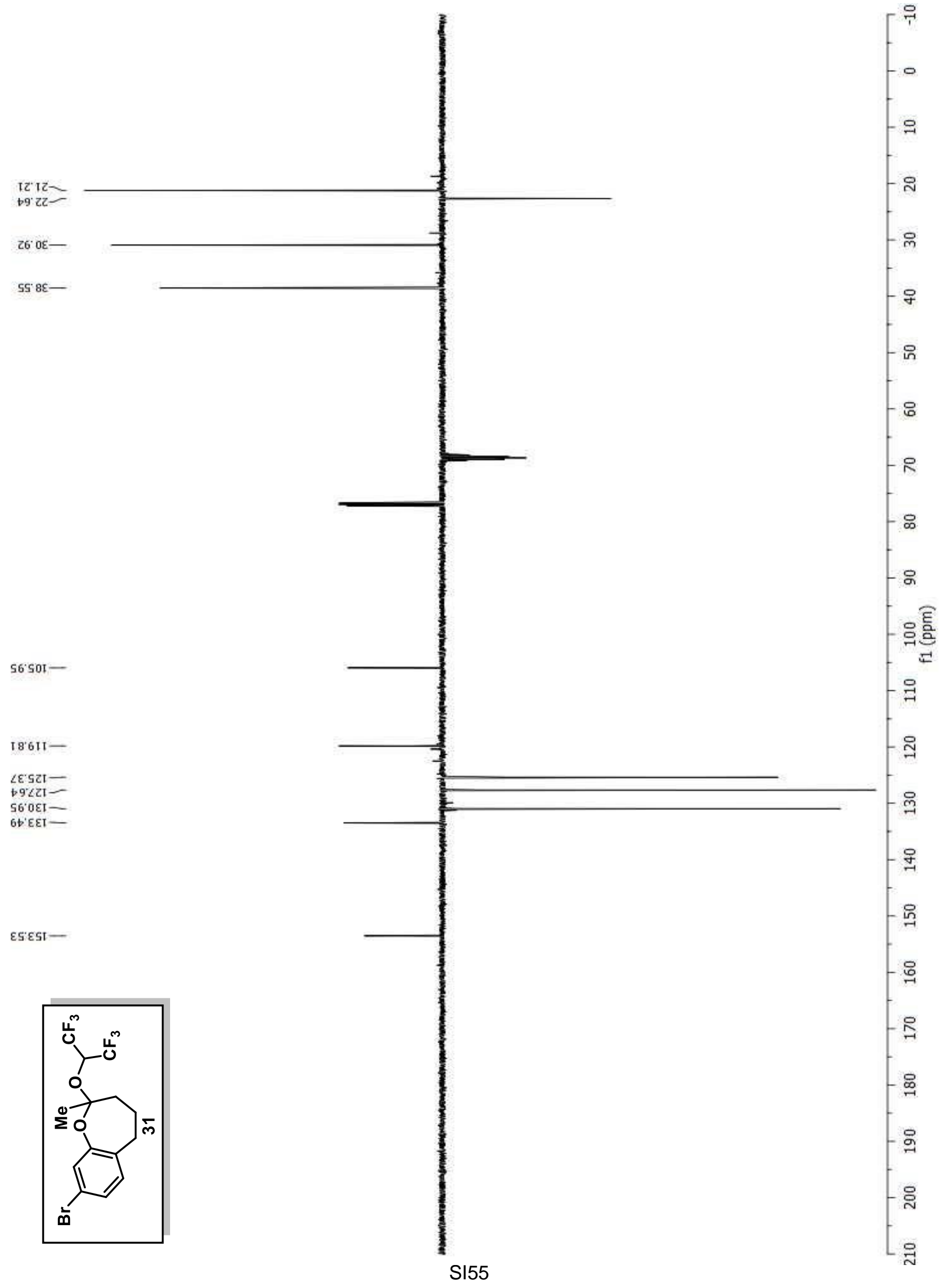




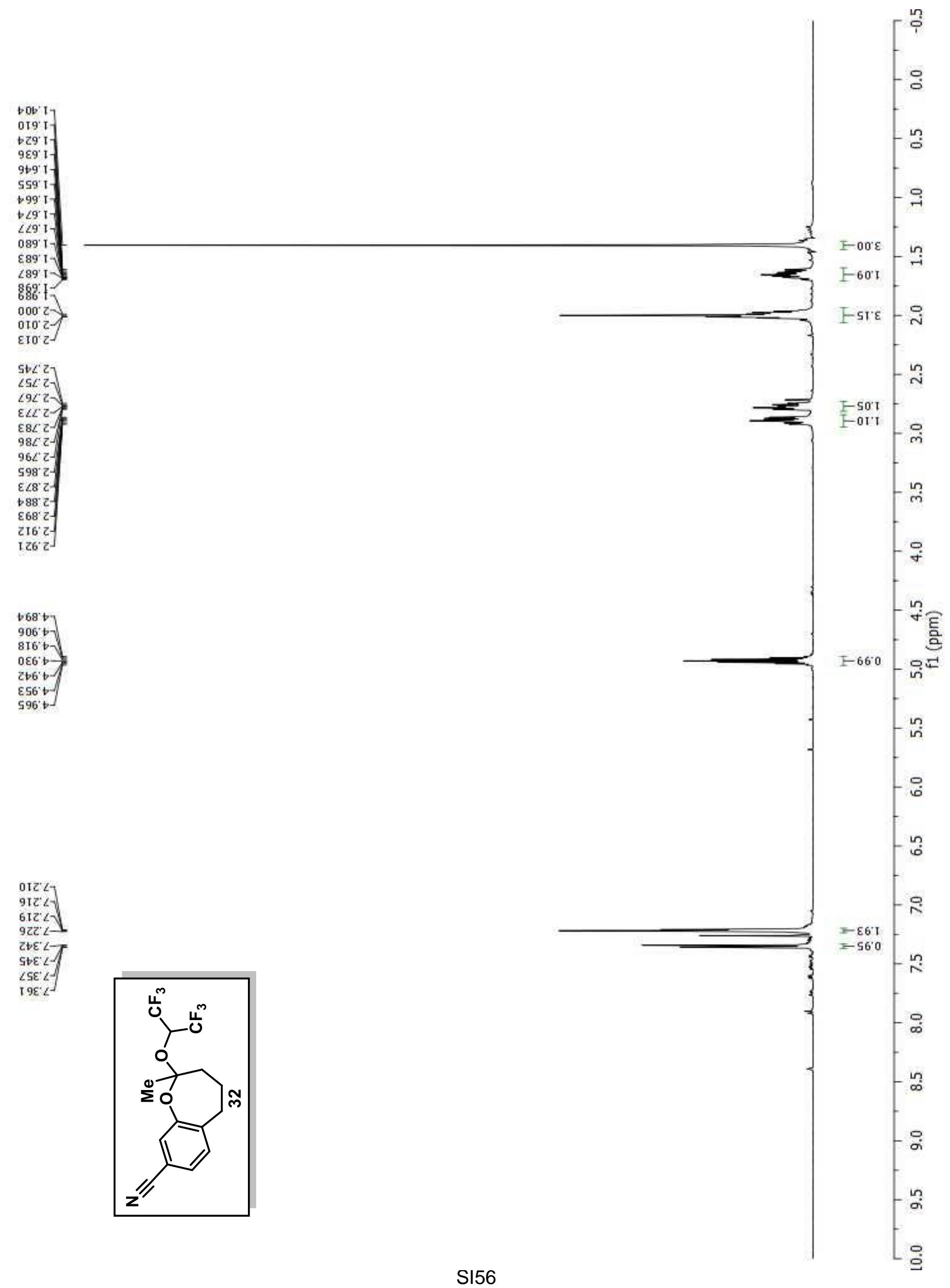




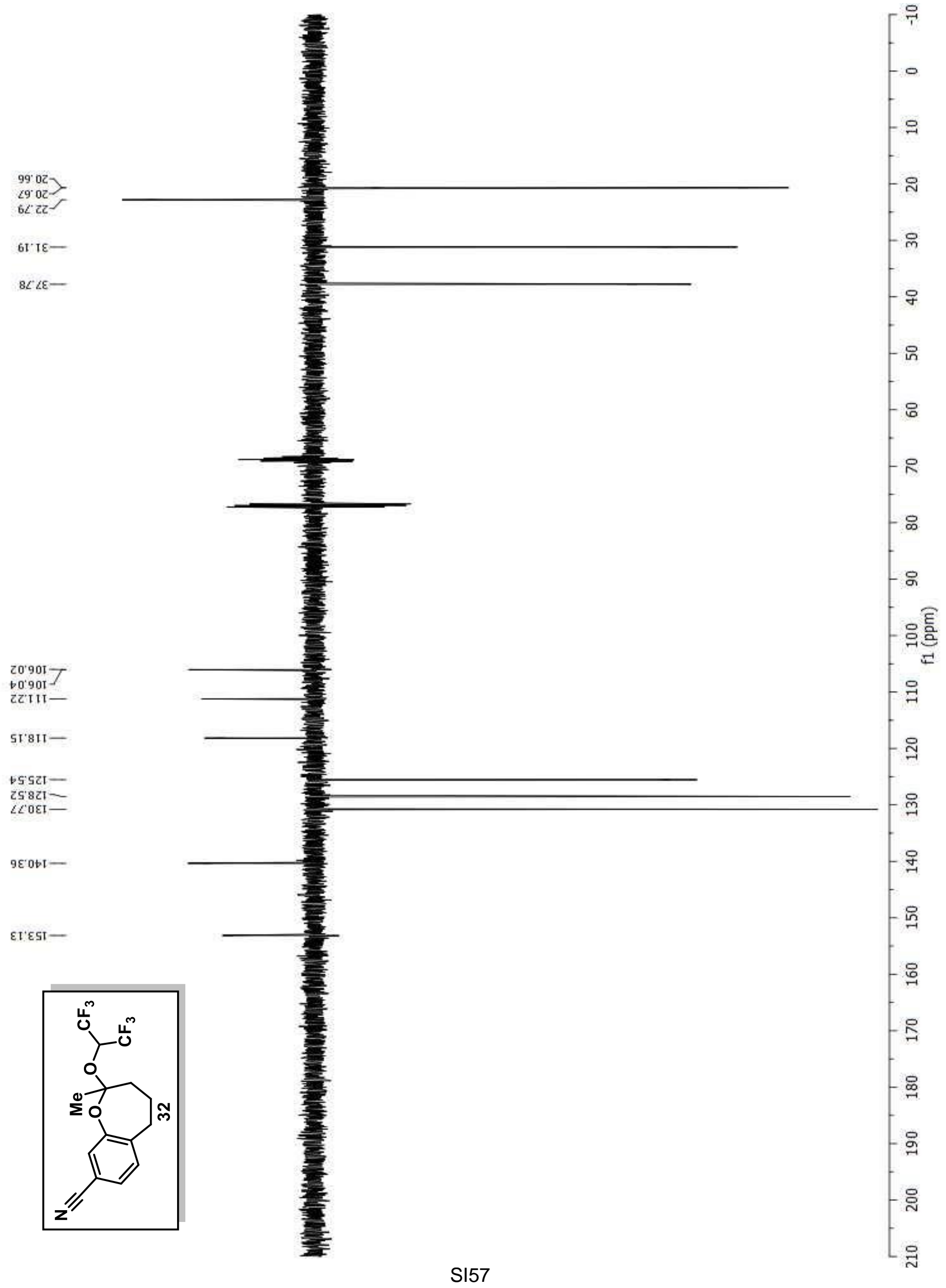



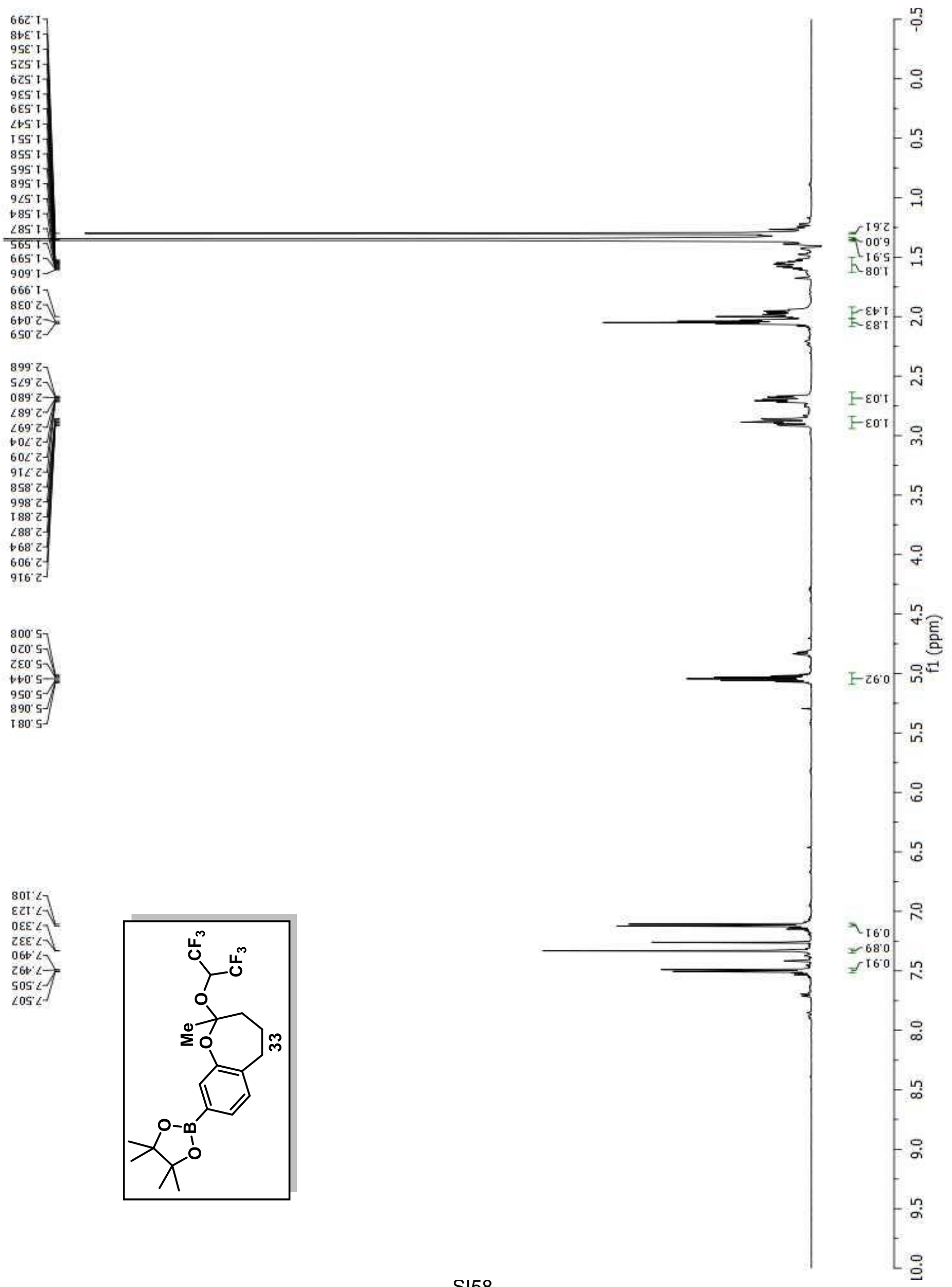


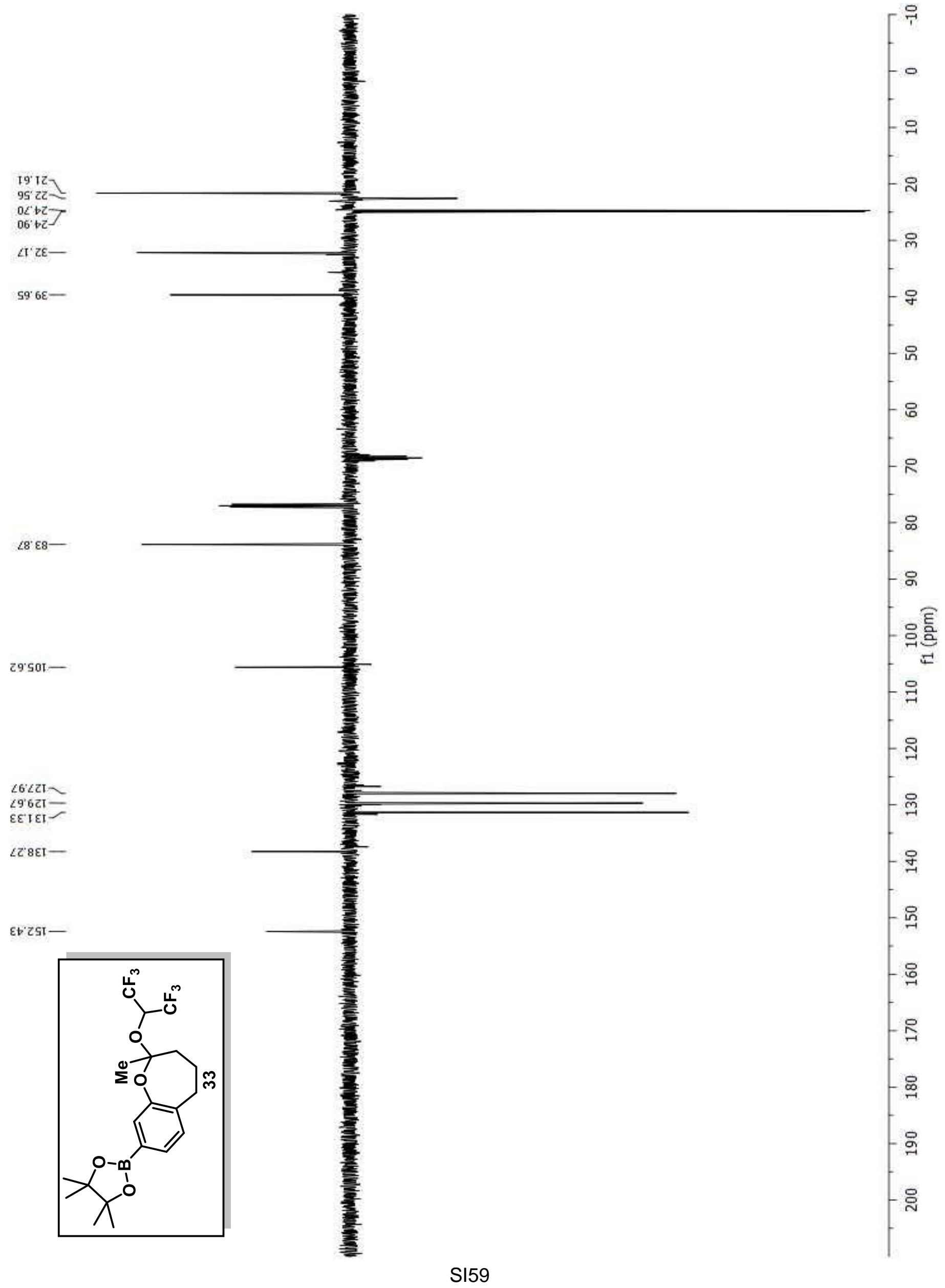




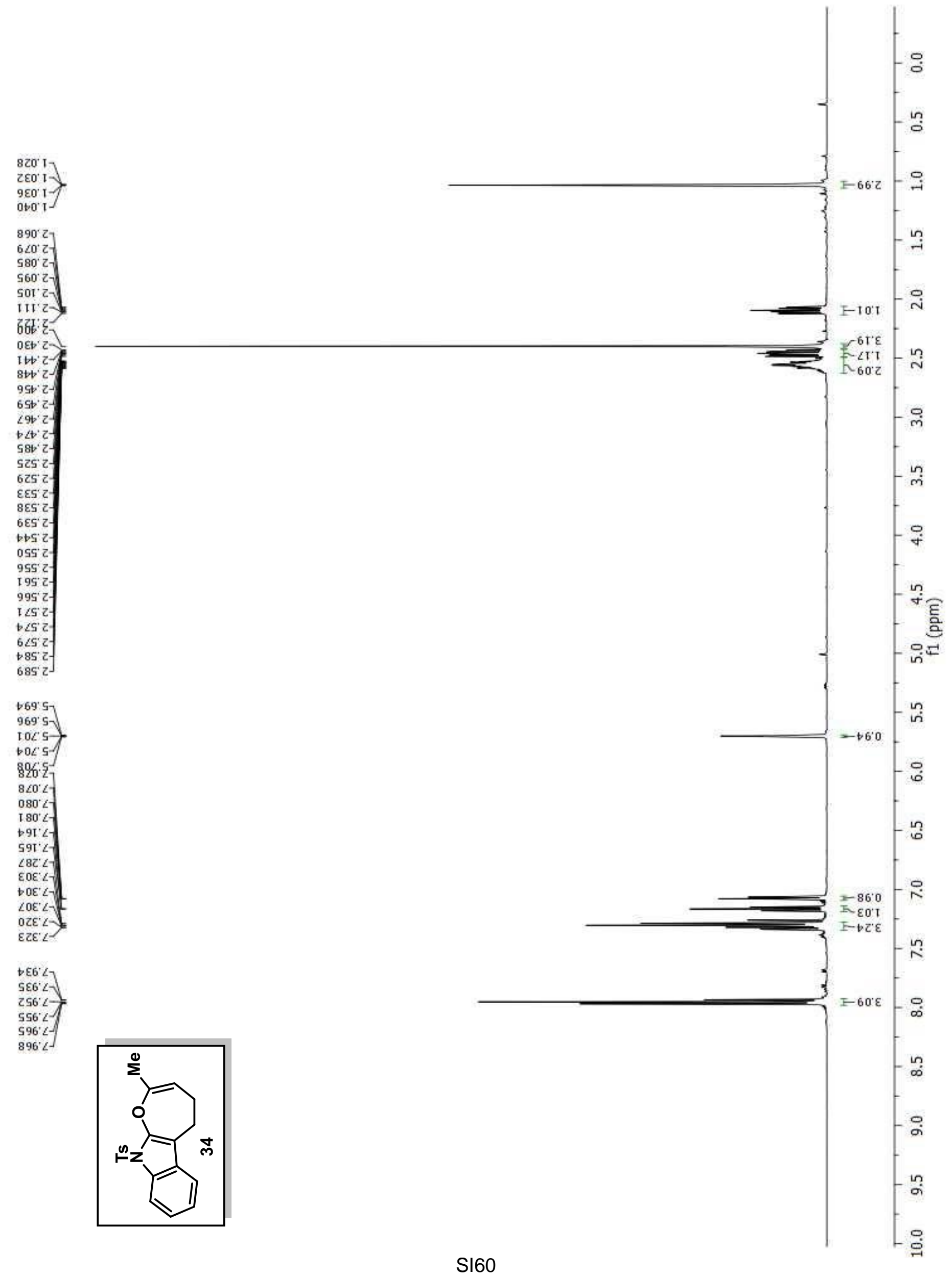




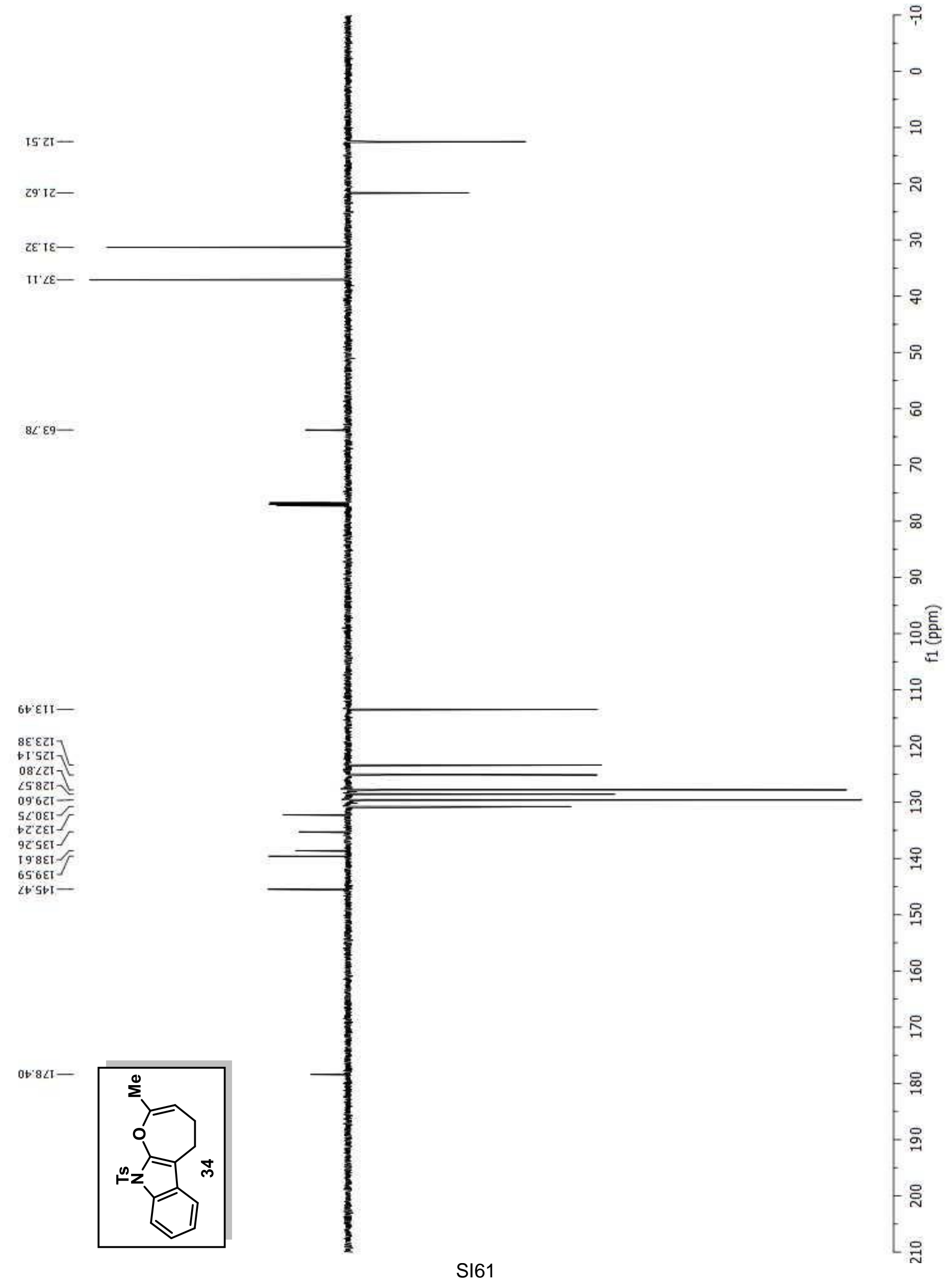




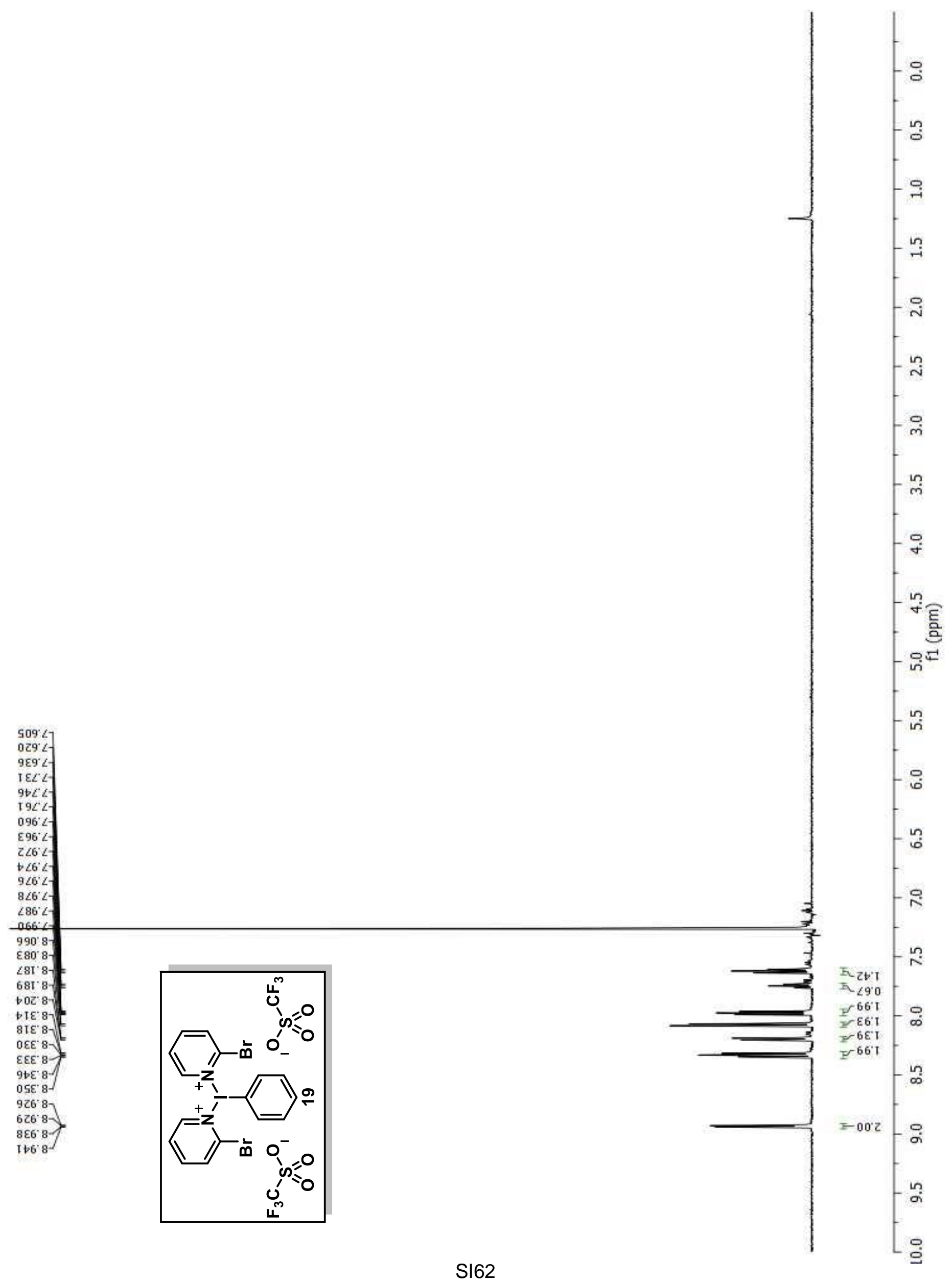




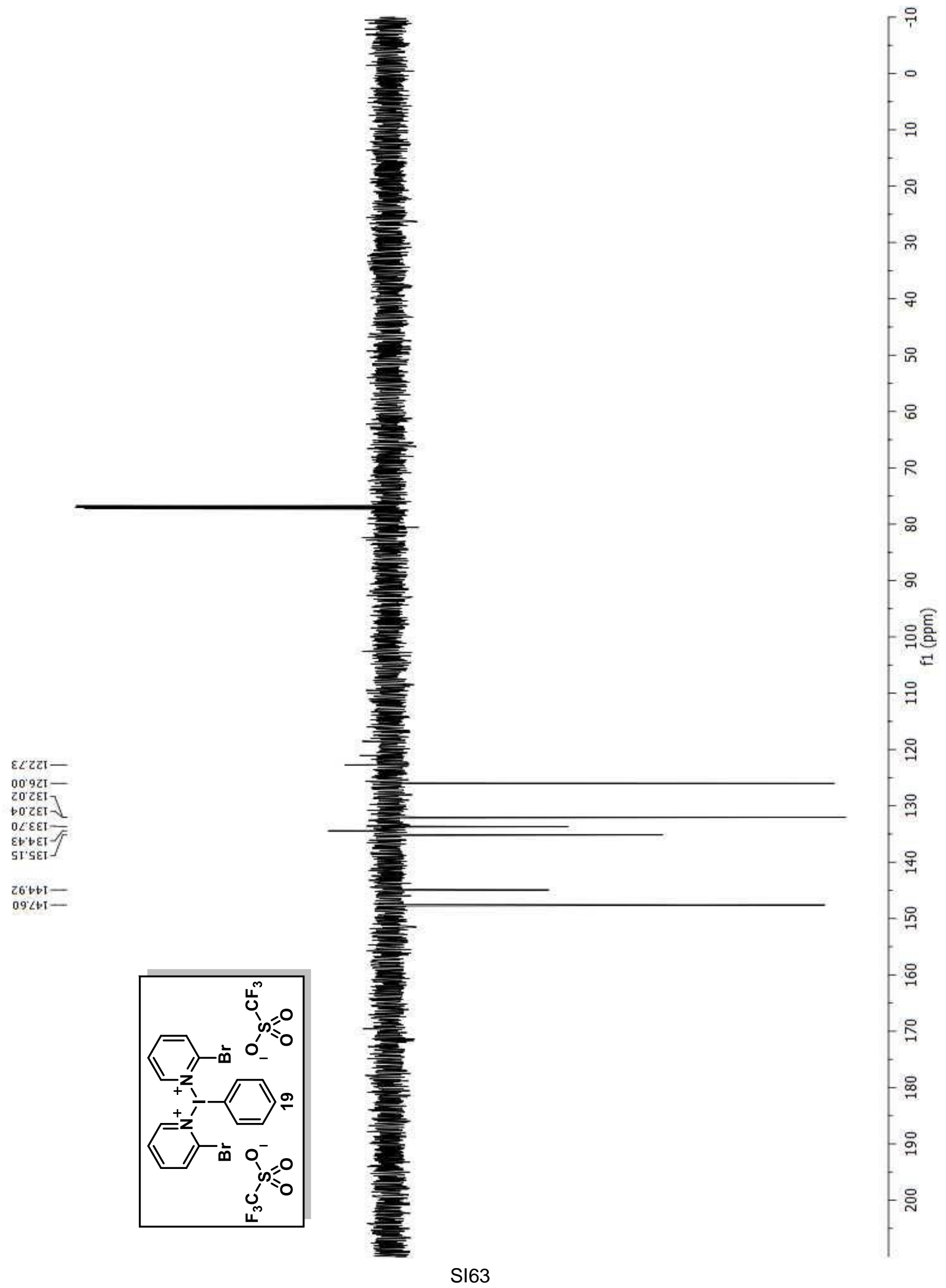


$9+8^{\circ} \mathrm{T}$

$878^{\circ} 1-$

$058 \mathrm{~T}-$

I S8: T-

¿ร8' I-

558' 1

$\angle E O^{\circ} \mathrm{Z}$

$0 \rightarrow 0$ Z

$z+0^{\circ} z$

$900 \mathrm{Z}$

$550 \mathrm{z}-\mathrm{y}$

$\angle S 0^{\circ} \mathrm{Z}$

$090^{\circ} \mathrm{Z}-$

$+90^{\circ} z-$

$890^{\circ} \mathrm{Z}$

$690^{\circ} \mathrm{Z}$

$\varepsilon \angle 0^{\circ} Z^{J}$

$\angle 8 \angle Z$

$66<2>$

I 18 '

$85 b$ b

$\angle 9 b^{\circ} b-$

$94 b^{\circ}$

$\angle b 8: 9$

058.

298:9-

$598 \cdot 9$

$5 \angle 8: 9$

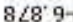

088' 9

068:97

$8+6.9$

606.9

C56.

$656.9-$

$596^{\circ} 9-$

$2 \angle 6: 9-$

$8<6.9-$

$I \rightarrow 0^{\circ} \angle$

$\varepsilon+0^{\circ}<$

$650^{\circ}<$

$09 \mathrm{I}^{\circ} \mathrm{C}$

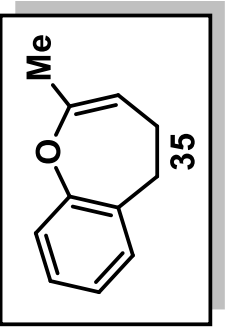

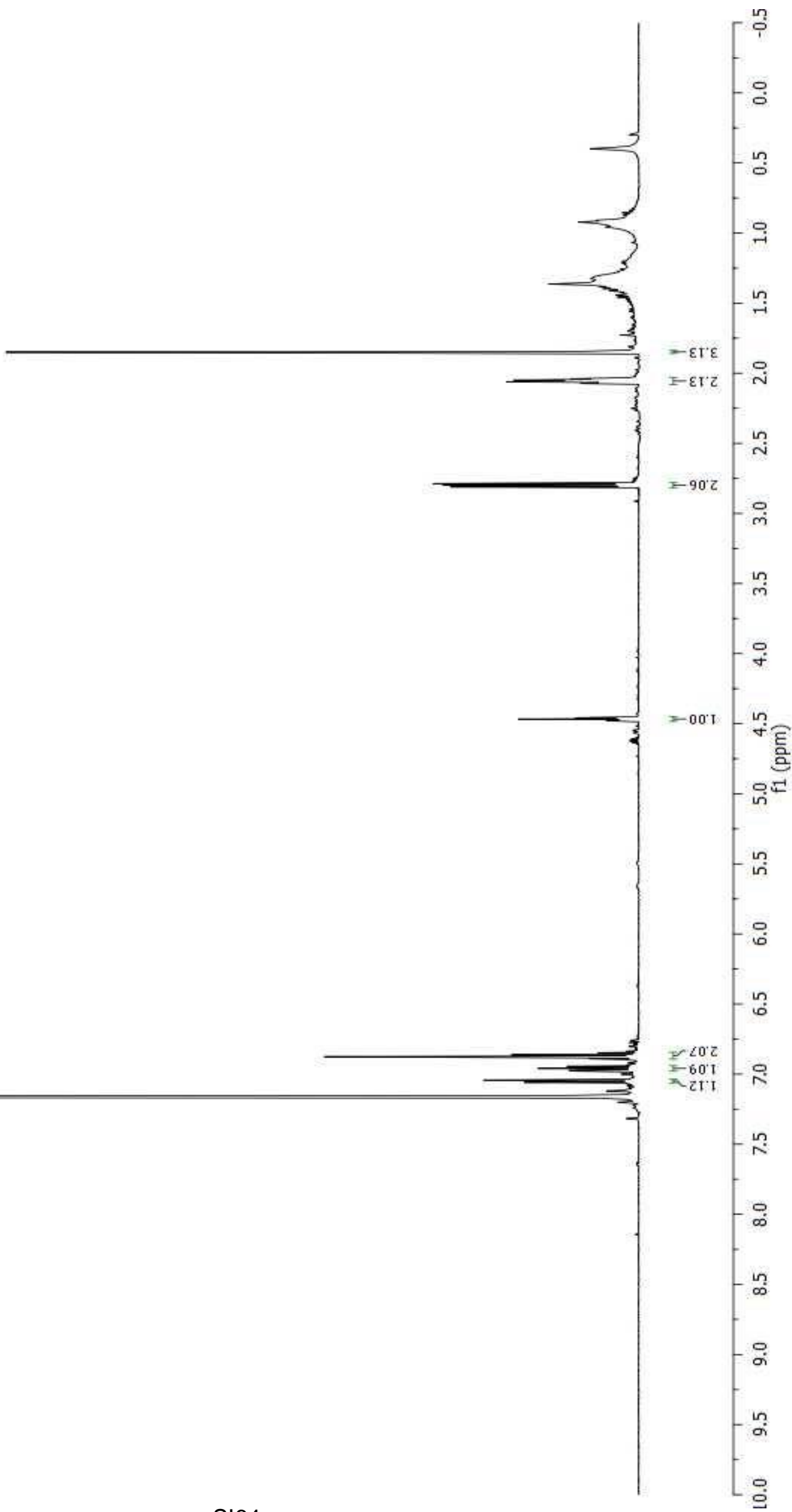




$$
\text { it }
$$




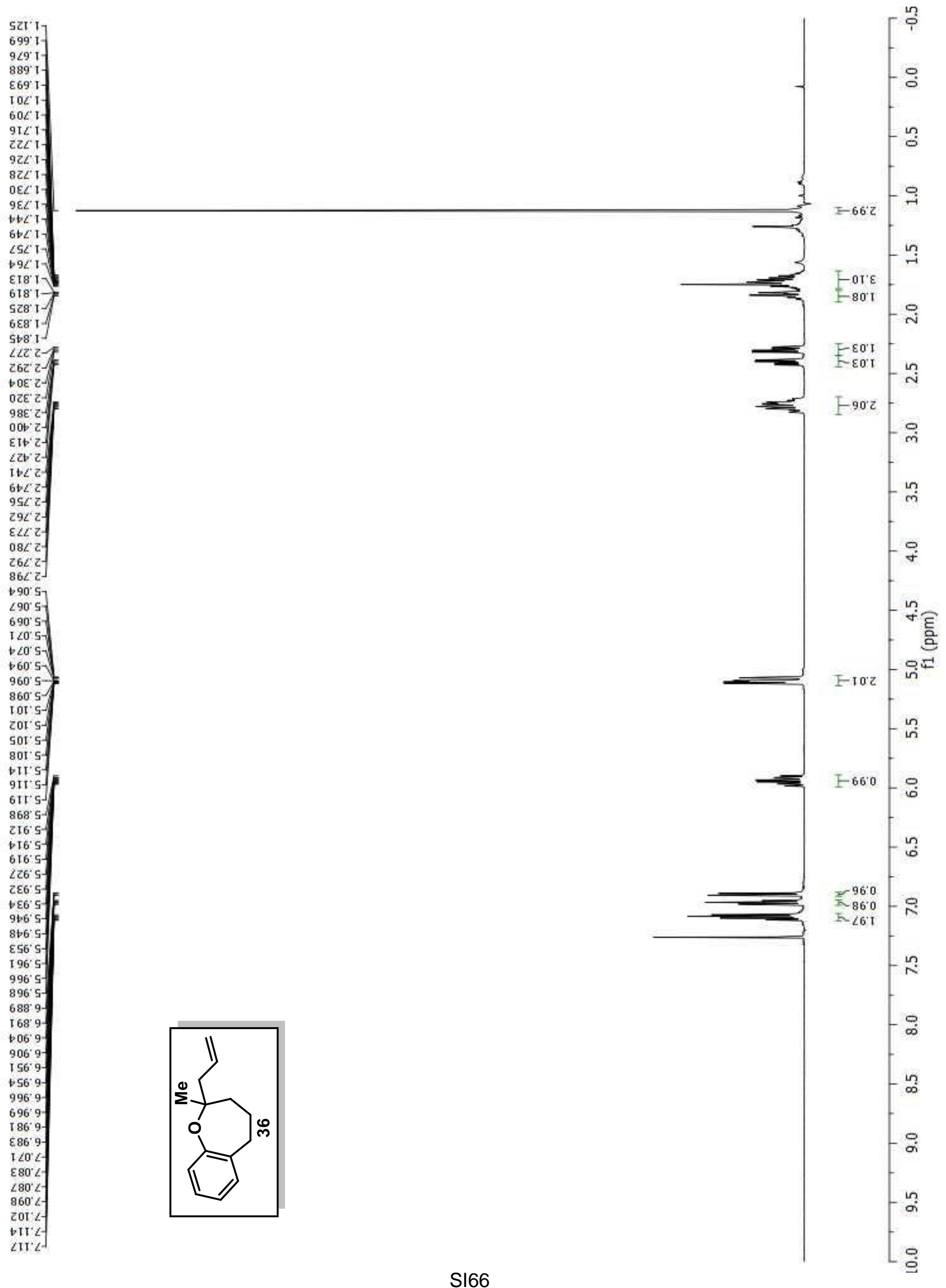




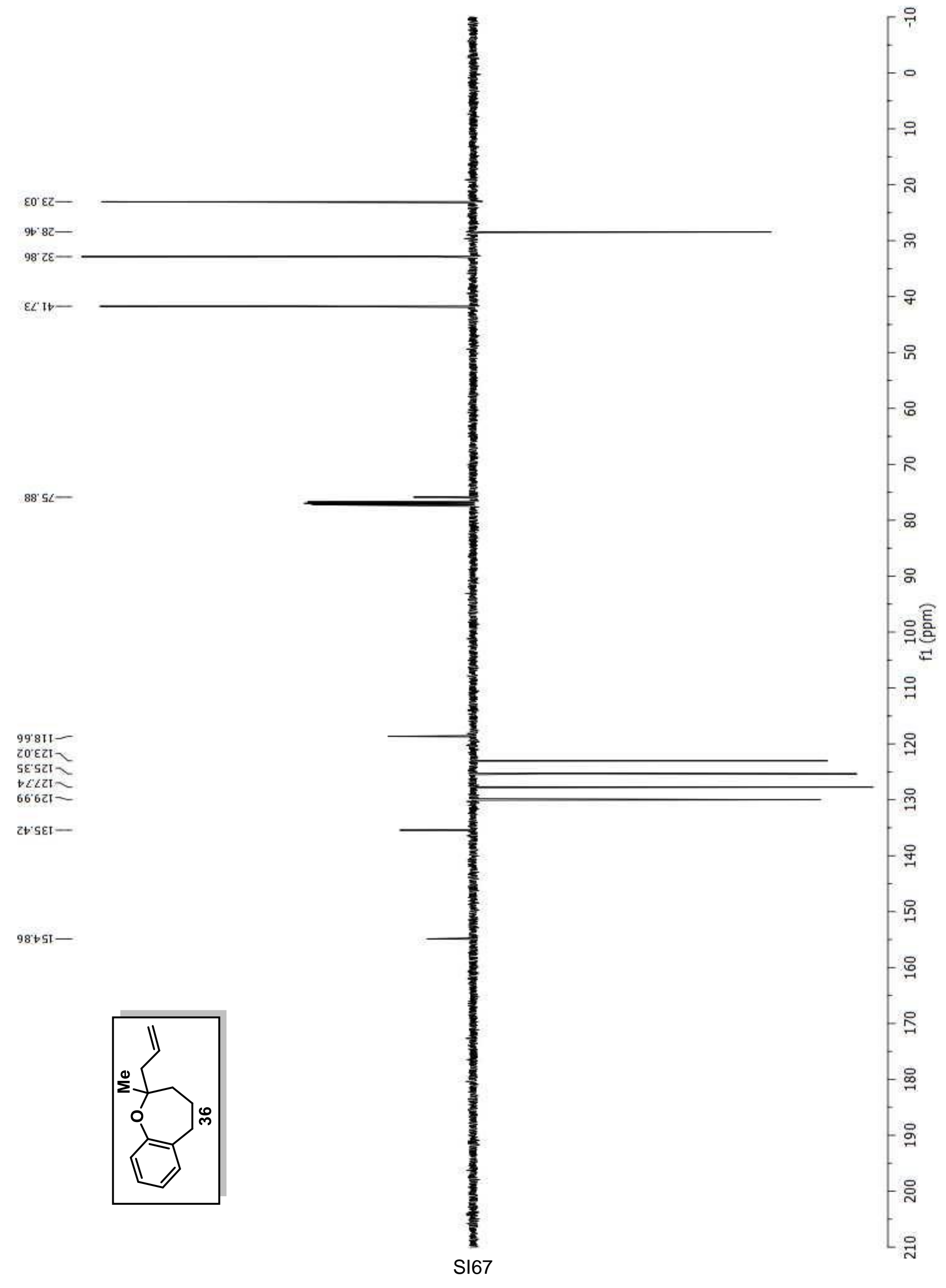




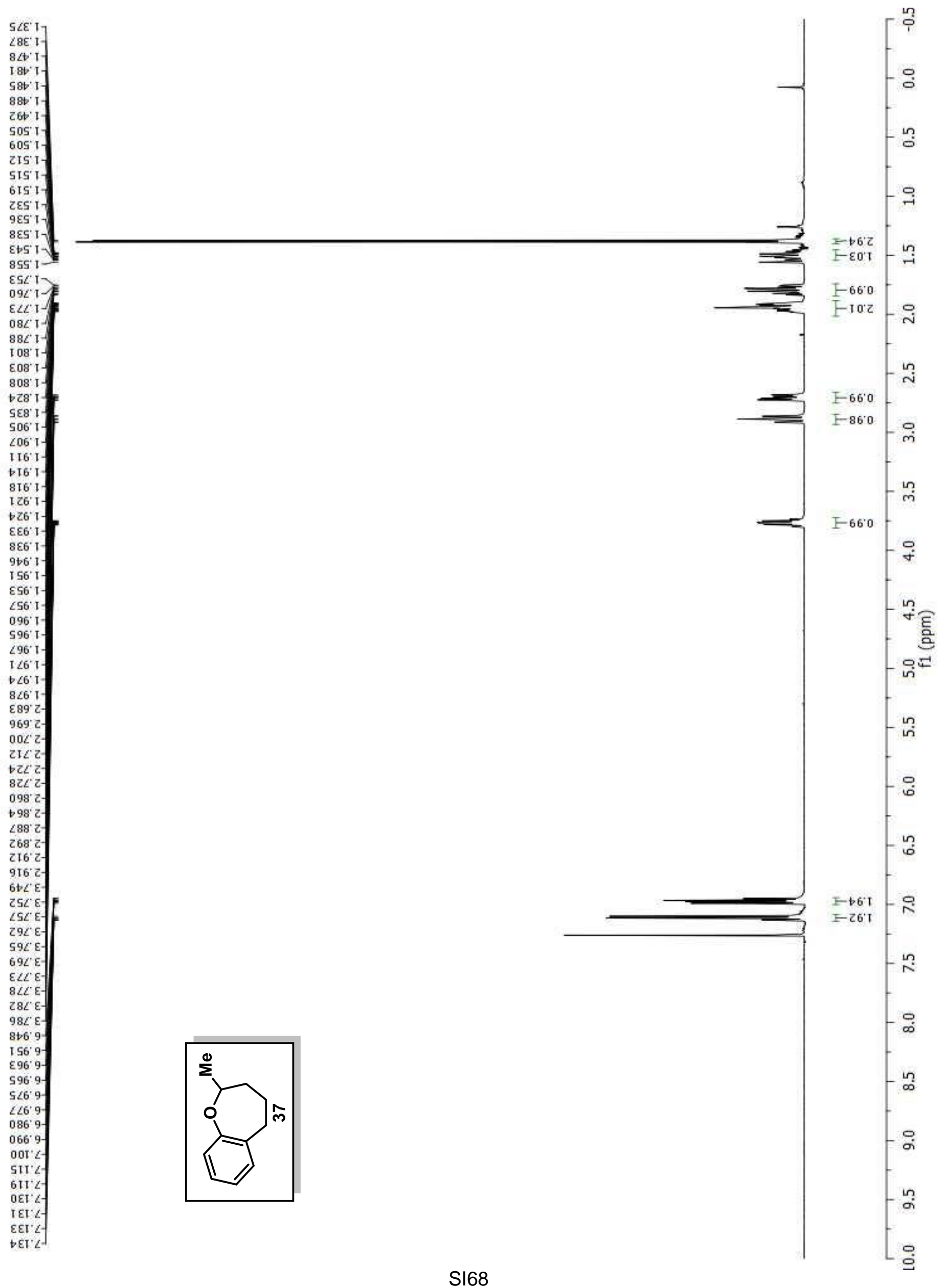



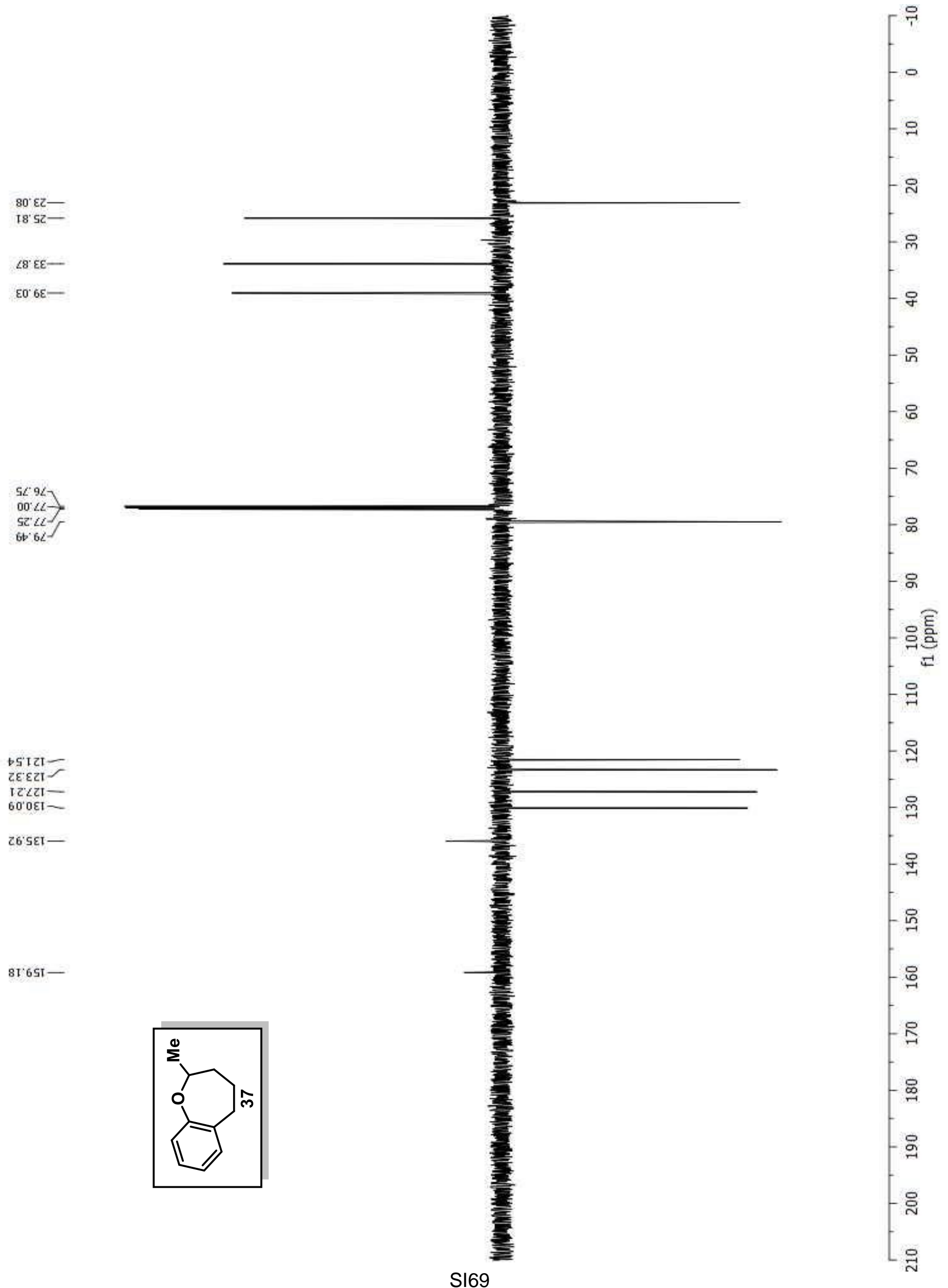


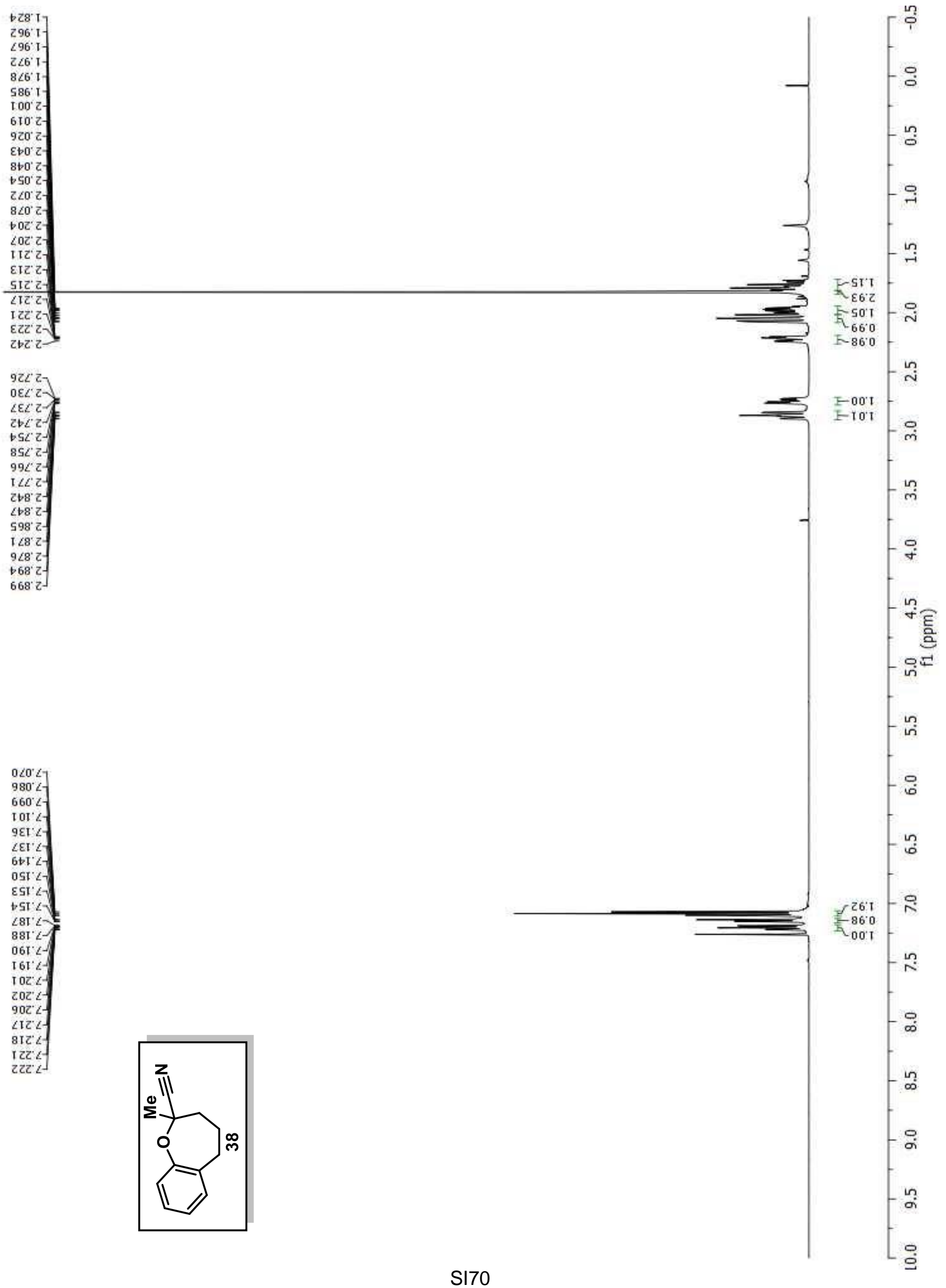




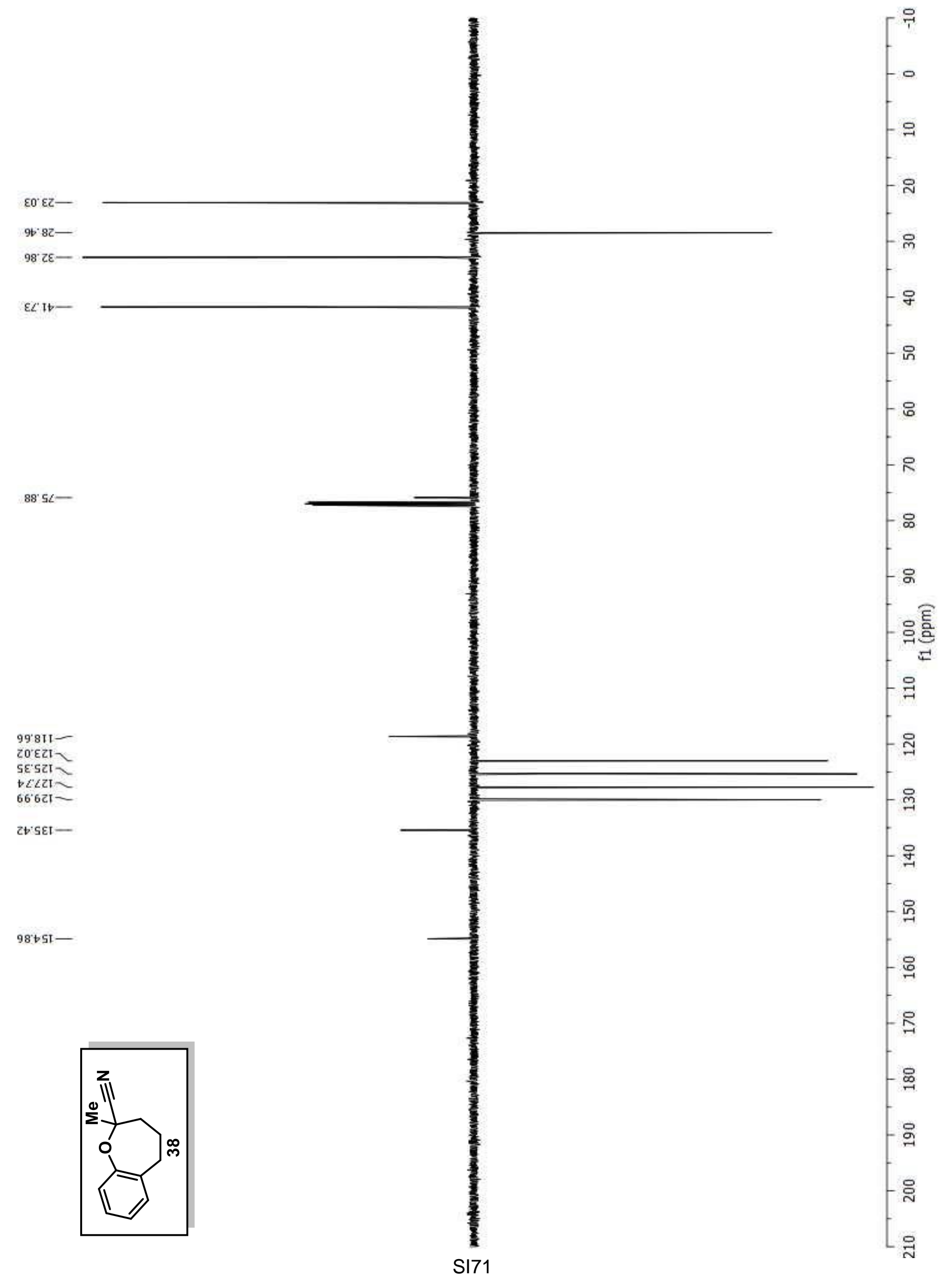




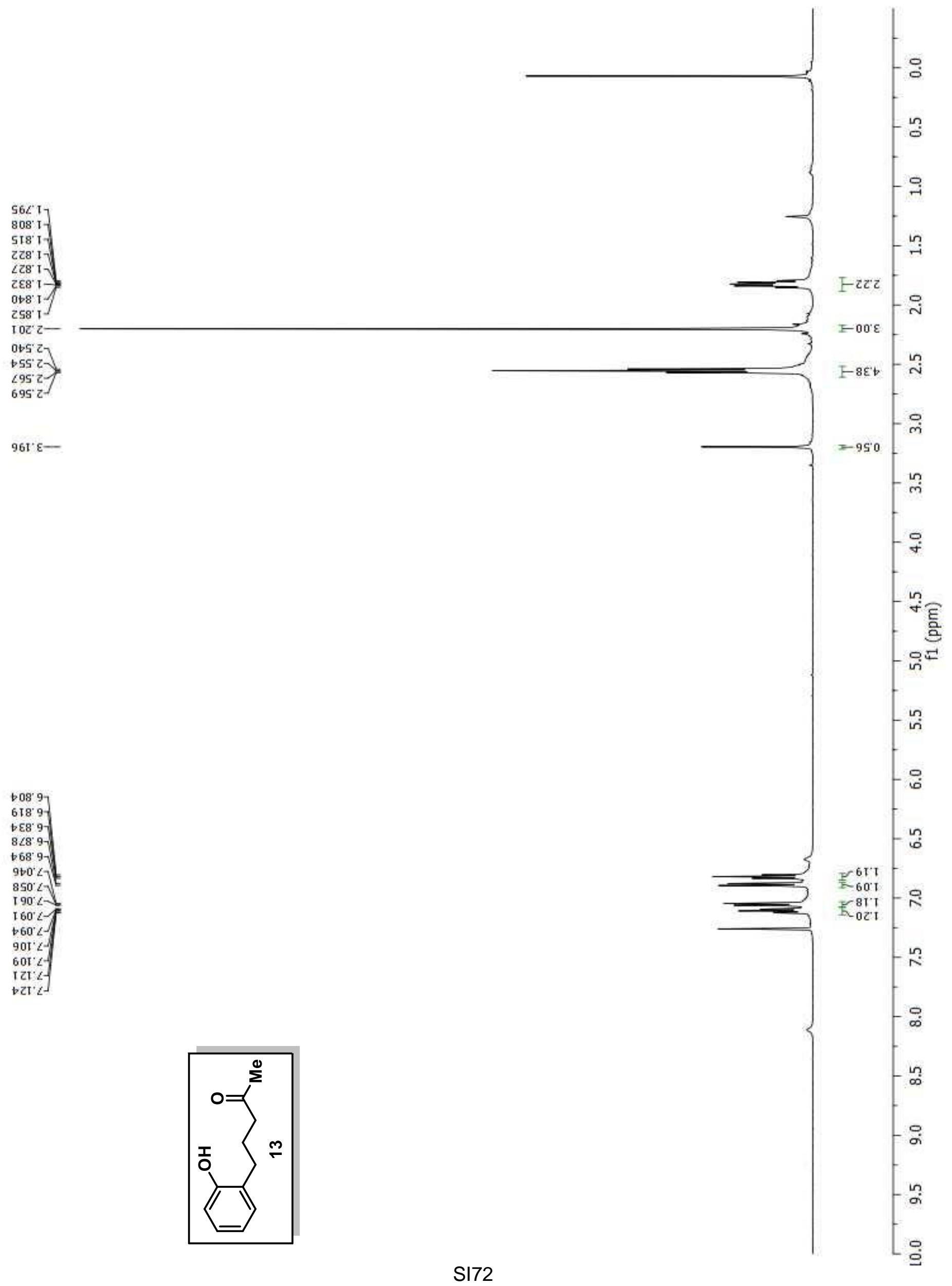




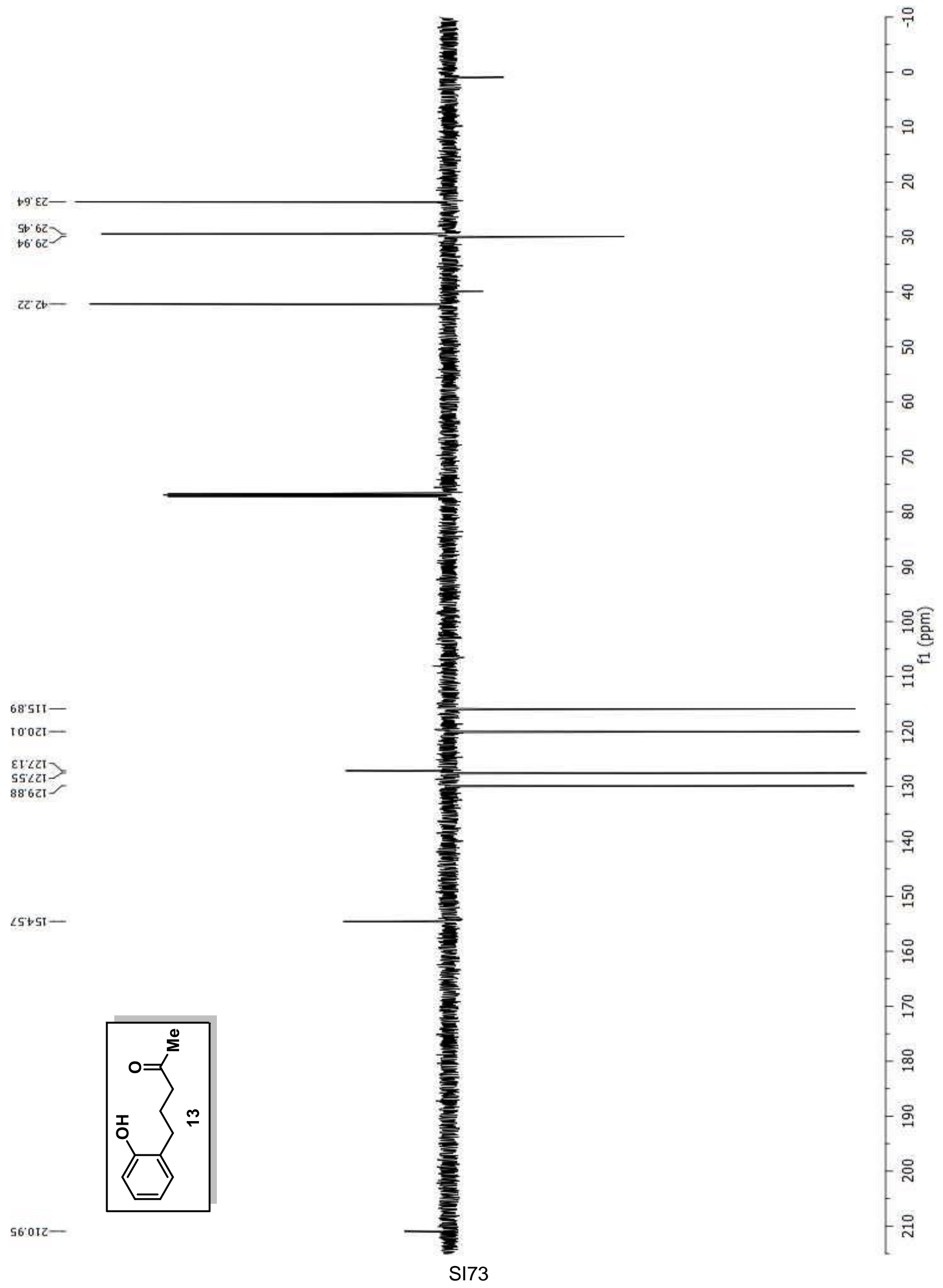




\section{Crystal Structure Report for 22}

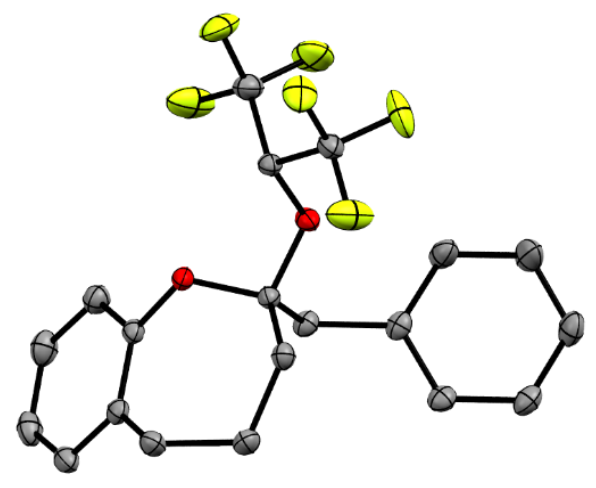

Figure 1. Crystal structure of $\mathrm{C}_{20} \mathrm{H}_{18} \mathrm{~F}_{6} \mathrm{O}_{2}$. Hydrogens have been omitted for clarity. Ellipsoids are shown as $30 \%$ occupancy.

A specimen of $\mathrm{C}_{20} \mathrm{H}_{18} \mathrm{~F}_{6} \mathrm{O}_{2}$ was used for the $\mathrm{X}$-ray crystallographic analysis. The X-ray intensity data were measured. The integration of the data using a triclinic unit cell yielded a total of 16257 reflections to a maximum $\theta$ angle of $27.89^{\circ}$ $\left(0.76 \AA\right.$ resolution), of which 4394 were independent (average redundancy 3.700 , completeness $=99.2 \%$, $R_{\text {int }}=$ $\left.3.33 \%, R_{\text {sig }}=2.97 \%\right)$ and $3623(82.45 \%)$ were greater than $2 \sigma\left(F^{2}\right)$. The final cell constants of $\underline{a}=9.2002(9) \AA$ 9.5076(9) $\AA, \underline{c}=11.4762(11) \AA, \alpha=101.253(2)^{\circ}, \beta=101.453(2)^{\circ}, \gamma=103.530(2)^{\circ}$, volume $=925.30(15) \AA^{3}$, are based upon the refinement of the XYZ-centroids of reflections above $20 \sigma(\mathrm{I})$.

The final anisotropic full-matrix least-squares refinement on $\mathrm{F}^{2}$ with 253 variables converged at $\mathrm{R} 1=3.92 \%$, for the observed data and $w R 2=9.77 \%$ for all data. The goodness-of-fit was 1.053 . The largest peak in the final difference electron density synthesis was $0.354 \mathrm{e}^{-} / \AA^{3}$ and the largest hole was $-0.370 \mathrm{e}^{-/} / \AA^{3}$ with an RMS deviation of $0.045 \mathrm{e}^{-/} \AA^{3}$. On the basis of the final model, the calculated density was $1.451 \mathrm{~g} / \mathrm{cm}^{3}$ and $F(000), 416 \mathrm{e}^{-}$.

\section{Table 1. Sample and crystal data for 22.}

Identification code
Chemical formula
Formula weight
Temperature
Wavelength
Crystal system
Space group
Unit cell dimensions

Volume

Z

Density (calculated)

Absorption coefficient

$F(000)$
2938

$\mathrm{C}_{20} \mathrm{H}_{18} \mathrm{~F}_{6} \mathrm{O}_{2}$

$404.34 \mathrm{~g} / \mathrm{mol}$

$100(2) \mathrm{K}$

$0.71073 \AA$

triclinic

$\mathrm{P}-1$

$\mathrm{a}=9.2002(9) \AA$

$\alpha=101.253(2)^{\circ}$

$\mathrm{b}=9.5076(9) \AA$

$\beta=101.453(2)^{\circ}$

$\mathrm{c}=11.4762(11) \AA$

925.30(15) $\AA^{3}$

2

$1.451 \mathrm{~g} / \mathrm{cm}^{3}$

$0.133 \mathrm{~mm}^{-1}$

416 


\section{Table 2. Data collection and structure refinement for 22.}

Theta range for data collection

Index ranges

Reflections collected

Independent reflections

Refinement method

Refinement program

Function minimized

Data / restraints / parameters

Goodness-of-fit on $\mathrm{F}^{2}$

Final $\mathbf{R}$ indices

\section{Weighting scheme}

Largest diff. peak and hole

R.M.S. deviation from mean
1.87 to $27.89^{\circ}$

$-12<=\mathrm{h}<=12,-11<=\mathrm{k}<=12,-15<=\mathrm{k}<=15$

16257

$4394[\mathrm{R}$ (int) $=0.0333]$

Full-matrix least-squares on $\mathrm{F}^{2}$

SHELXL-2014/7 (Sheldrick, 2014)

$\Sigma \mathrm{w}\left(\mathrm{F}_{\mathrm{o}}{ }^{2}-\mathrm{F}_{\mathrm{c}}{ }^{2}\right)^{2}$

4394 / 0 / 253

1.053

3623 data; $\mid>2 \sigma(I) \quad R 1=0.0392, w R 2=0.0916$

all data

$\mathrm{R} 1=0.0489, \mathrm{wR} 2=0.0977$

$w=1 /\left[\sigma^{2}\left(F_{o}^{2}\right)+(0.0372 P)^{2}+0.3757 P\right]$

where $\mathrm{P}=\left(\mathrm{F}_{\mathrm{o}}{ }^{2}+2 \mathrm{~F}_{\mathrm{c}}{ }^{2}\right) / 3$

0.354 and $-0.370 \mathrm{e}^{-3}$

$0.045 \mathrm{e}^{-3}$

\section{Table 3. Atomic coordinates and equivalent isotropic atomic displacement parameters $\left(\AA^{2}\right)$ for 22.}

$\mathrm{U}(\mathrm{eq})$ is defined as one third of the trace of the orthogonalized $\mathrm{U}_{\mathrm{ij}}$ tensor.

\begin{tabular}{lllll} 
& \multicolumn{1}{c}{$\mathbf{x} / \mathbf{a}$} & \multicolumn{1}{c}{$\mathbf{y} / \mathbf{b}$} & $\mathbf{z} / \mathbf{c}$ & $\mathbf{U}(\mathbf{e q})$ \\
F1 & $0.16350(10)$ & $0.98914(9)$ & $0.04806(8)$ & $0.0339(2)$ \\
F2 & $0.29144(12)$ & $0.98786(10)$ & $0.22478(9)$ & $0.0420(3)$ \\
F3 & $0.40529(11)$ & $0.00659(10)$ & $0.08148(10)$ & $0.0434(3)$ \\
F4 & $0.07970(9)$ & $0.21697(11)$ & $0.15925(9)$ & $0.0380(2)$ \\
F5 & $0.22276(11)$ & $0.23584(13)$ & $0.33639(9)$ & $0.0429(3)$ \\
F6 & $0.25388(11)$ & $0.42168(10)$ & $0.25480(11)$ & $0.0477(3)$ \\
O1 & $0.48439(10)$ & $0.27335(10)$ & $0.25708(8)$ & $0.01802(19)$ \\
O2 & $0.54088(9)$ & $0.47700(10)$ & $0.18017(8)$ & $0.01713(19)$ \\
C1 & $0.34076(13)$ & $0.22012(14)$ & $0.16990(11)$ & $0.0165(2)$ \\
C2 & $0.29947(15)$ & $0.04956(15)$ & $0.13128(12)$ & $0.0212(3)$ \\
C3 & $0.22370(15)$ & $0.27385(16)$ & $0.23089(13)$ & $0.0244(3)$ \\
C4 & $0.60991(13)$ & $0.37503(14)$ & $0.23240(11)$ & $0.0156(2)$ \\
C5 & $0.67924(14)$ & $0.29177(14)$ & $0.14126(11)$ & $0.0179(3)$ \\
C6 & $0.81645(14)$ & $0.38836(15)$ & $0.10930(12)$ & $0.0202(3)$ \\
C7 & $0.77816(15)$ & $0.50807(15)$ & $0.04629(12)$ & $0.0202(3)$ \\
C8 & $0.75079(14)$ & $0.63902(14)$ & $0.12805(11)$ & $0.0179(3)$ \\
C9 & $0.63427(14)$ & $0.61958(14)$ & $0.19020(11)$ & $0.0168(2)$ \\
C10 & $0.60038(16)$ & $0.73972(15)$ & $0.25772(12)$ & $0.0224(3)$
\end{tabular}




\begin{tabular}{lllll} 
& \multicolumn{1}{c}{$\mathbf{x} / \mathbf{a}$} & $\mathbf{y} / \mathbf{b}$ & $\mathbf{z} / \mathbf{c}$ & $\mathbf{U}(\mathbf{e q})$ \\
$\mathrm{C} 11$ & $0.68609(17)$ & $0.88465(16)$ & $0.26730(13)$ & $0.0264(3)$ \\
$\mathrm{C} 12$ & $0.80547(16)$ & $0.90738(15)$ & $0.21016(13)$ & $0.0262(3)$ \\
$\mathrm{C} 13$ & $0.83705(15)$ & $0.78601(15)$ & $0.14169(12)$ & $0.0223(3)$ \\
$\mathrm{C} 14$ & $0.71923(14)$ & $0.45250(14)$ & $0.36040(11)$ & $0.0179(3)$ \\
$\mathrm{C} 15$ & $0.76713(14)$ & $0.34486(14)$ & $0.43121(11)$ & $0.0183(3)$ \\
$\mathrm{C} 16$ & $0.67263(16)$ & $0.27869(17)$ & $0.49835(12)$ & $0.0262(3)$ \\
$\mathrm{C} 17$ & $0.71623(18)$ & $0.18125(19)$ & $0.56471(13)$ & $0.0317(3)$ \\
$\mathrm{C} 18$ & $0.85632(17)$ & $0.14930(17)$ & $0.56614(13)$ & $0.0271(3)$ \\
$\mathrm{C} 19$ & $0.95154(15)$ & $0.21392(15)$ & $0.49991(12)$ & $0.0230(3)$ \\
$\mathrm{C} 20$ & $0.90686(14)$ & $0.31108(15)$ & $0.43278(12)$ & $0.0204(3)$
\end{tabular}

\section{Table 4. Bond lengths ( $(\AA)$ for 22.}

$\begin{array}{llll}\text { F1-C2 } & 1.3350(15) & \text { F2-C2 } & 1.3258(15) \\ \text { F3-C2 } & 1.3256(16) & \text { F4-C3 } & 1.3349(16) \\ \text { F5-C3 } & 1.3305(17) & \text { F6-C3 } & 1.3283(16) \\ \text { O1-C1 } & 1.4028(14) & \text { O1-C4 } & 1.4362(14) \\ \text { O2-C9 } & 1.3942(15) & \text { O2-C4 } & 1.4375(14) \\ \text { C1-C3 } & 1.5258(18) & \text { C1-C2 } & 1.5280(18) \\ \text { C1-H1 } & 1.0 & \text { C4-C5 } & 1.5184(18) \\ \text { C4-C14 } & 1.5349(16) & \text { C5-C6 } & 1.5311(18) \\ \text { C5-H5A } & 0.99 & \text { C5-H5B } & 0.99 \\ \text { C6-C7 } & 1.5330(18) & \text { C6-H6A } & 0.99 \\ \text { C6-H6B } & \text { C7-C8 } & 1.5095(18) \\ \text { C7-H7A } & 0.99 & \text { C7-H7B } & 0.99 \\ \text { C8-C13 } & 0.99 & \text { C8-C9 } & 1.3980(18) \\ \text { C9-C10 } & \text { C10-C11 } & 1.3895(19) \\ \text { C10-H10 } & 1.3978(18) & \mathrm{C} 11-\mathrm{C} 12 & 1.383(2) \\ \text { C11-H11 } & 1.3854(19) & \mathrm{C} 12-\mathrm{C} 13 & 1.388(2) \\ \text { C12-H12 } & 0.95 & \mathrm{C} 13-\mathrm{H} 13 & 0.95 \\ \mathrm{C} 14-\mathrm{C} 15 & \mathrm{C} 14-\mathrm{H} 14 \mathrm{~A} & 0.99 \\ \mathrm{C} 14-\mathrm{H} 14 \mathrm{~B} & 0.95 & \mathrm{C} 15-\mathrm{C} 20 & 1.3937(18) \\ \mathrm{C} 15-\mathrm{C} 16 & 0.95 & \mathrm{C} 16-\mathrm{C} 17 & 1.3883(19) \\ \mathrm{C} 16-\mathrm{H} 16 & 1.5150(17) & \mathrm{C} 17-\mathrm{C} 18 & 1.389(2) \\ \mathrm{C} 17-\mathrm{H} 17 & \mathrm{C} 18-\mathrm{C} 19 & 1.385(2) \\ \mathrm{C} 18-\mathrm{H} 18 & 1.3945(19) & \mathrm{C} 19-\mathrm{C} 20 & 1.3948(18) \\ \mathrm{C} 19-\mathrm{H} 19 & \mathrm{C} 20-\mathrm{H} 20 & 0.95 \\ & 0.95 & & \end{array}$

\section{Table 5. Bond angles $\left({ }^{\circ}\right)$ for 22.}

C1-O1-C4
O1-C1-C3
C3-C1-C2
C3-C1-H1
F3-C2-F2
F2-C2-F1

119.12(9)

107.21(10)

$111.89(10)$

109.8

107.03(11)

106.86(11)
$\mathrm{C} 9-\mathrm{O} 2-\mathrm{C} 4$

$\mathrm{O} 1-\mathrm{C} 1-\mathrm{C} 2$

$\mathrm{O} 1-\mathrm{C} 1-\mathrm{H} 1$

$\mathrm{C} 2-\mathrm{C} 1-\mathrm{H} 1$

$\mathrm{F} 3-\mathrm{C} 2-\mathrm{F} 1$

$\mathrm{F} 3-\mathrm{C} 2-\mathrm{C} 1$
118.61(9)

$108.15(10)$

109.8

109.8

$107.21(12)$

$110.38(10)$ 


\begin{tabular}{|c|c|c|c|}
\hline F2-C2-C1 & $112.86(11)$ & $\mathrm{F} 1-\mathrm{C} 2-\mathrm{C} 1$ & $112.19(10)$ \\
\hline F6-C3-F5 & $107.48(13)$ & F6-C3-F4 & $106.74(11)$ \\
\hline F5-C3-F4 & $106.84(11)$ & F6-C3-C1 & $111.20(11)$ \\
\hline F5-C3-C1 & $112.32(11)$ & F4-C3-C1 & $111.95(11)$ \\
\hline O1-C4-O2 & $104.69(9)$ & O1-C4-C5 & $110.57(10)$ \\
\hline O2-C4-C5 & $110.33(10)$ & $\mathrm{O} 1-\mathrm{C} 4-\mathrm{C} 14$ & $103.49(9)$ \\
\hline $\mathrm{O} 2-\mathrm{C} 4-\mathrm{C} 14$ & $111.56(10)$ & C5-C4-C14 & $115.45(10)$ \\
\hline C4-C5-C6 & $115.42(11)$ & $\mathrm{C} 4-\mathrm{C} 5-\mathrm{H} 5 \mathrm{~A}$ & 108.4 \\
\hline C6-C5-H5A & 108.4 & C4-C5-H5B & 108.4 \\
\hline C6-C5-H5B & 108.4 & H5A-C5-H5B & 107.5 \\
\hline $\mathrm{C} 5-\mathrm{C} 6-\mathrm{C} 7$ & $114.46(10)$ & $\mathrm{C} 5-\mathrm{C} 6-\mathrm{H} 6 \mathrm{~A}$ & 108.6 \\
\hline C7-C6-H6A & 108.6 & $\mathrm{C} 5-\mathrm{C} 6-\mathrm{H} 6 \mathrm{~B}$ & 108.6 \\
\hline C7-C6-H6B & 108.6 & $\mathrm{H} 6 \mathrm{~A}-\mathrm{C} 6-\mathrm{H} 6 \mathrm{~B}$ & 107.6 \\
\hline C8-C7-C6 & $115.43(10)$ & $\mathrm{C} 8-\mathrm{C} 7-\mathrm{H} 7 \mathrm{~A}$ & 108.4 \\
\hline $\mathrm{C} 6-\mathrm{C} 7-\mathrm{H} 7 \mathrm{~A}$ & 108.4 & $\mathrm{C} 8-\mathrm{C} 7-\mathrm{H} 7 \mathrm{~B}$ & 108.4 \\
\hline $\mathrm{C} 6-\mathrm{C} 7-\mathrm{H} 7 \mathrm{~B}$ & 108.4 & H7A-C7-H7B & 107.5 \\
\hline C13-C8-C9 & $116.79(12)$ & $\mathrm{C} 13-\mathrm{C} 8-\mathrm{C} 7$ & $121.55(12)$ \\
\hline $\mathrm{C} 9-\mathrm{C} 8-\mathrm{C} 7$ & $121.62(11)$ & C10-C9-O2 & $117.21(11)$ \\
\hline C10-C9-C8 & $122.01(12)$ & O2-C9-C8 & $120.66(11)$ \\
\hline C9-C10-C11 & $119.77(13)$ & $\mathrm{C} 9-\mathrm{C} 10-\mathrm{H} 10$ & 120.1 \\
\hline $\mathrm{C} 11-\mathrm{C} 10-\mathrm{H} 10$ & 120.1 & C12-C11-C10 & $119.56(13)$ \\
\hline $\mathrm{C} 12-\mathrm{C} 11-\mathrm{H} 11$ & 120.2 & $\mathrm{C} 10-\mathrm{C} 11-\mathrm{H} 11$ & 120.2 \\
\hline C11-C12-C13 & $120.05(13)$ & $\mathrm{C} 11-\mathrm{C} 12-\mathrm{H} 12$ & 120.0 \\
\hline $\mathrm{C} 13-\mathrm{C} 12-\mathrm{H} 12$ & 120.0 & C12-C13-C8 & $121.76(13)$ \\
\hline $\mathrm{C} 12-\mathrm{C} 13-\mathrm{H} 13$ & 119.1 & $\mathrm{C} 8-\mathrm{C} 13-\mathrm{H} 13$ & 119.1 \\
\hline C15-C14-C4 & $113.68(10)$ & C15-C14-H14A & 108.8 \\
\hline $\mathrm{C} 4-\mathrm{C} 14-\mathrm{H} 14 \mathrm{~A}$ & 108.8 & C15-C14-H14B & 108.8 \\
\hline $\mathrm{C} 4-\mathrm{C} 14-\mathrm{H} 14 \mathrm{~B}$ & 108.8 & $\mathrm{H} 14 \mathrm{~A}-\mathrm{C} 14-\mathrm{H} 14 \mathrm{~B}$ & 107.7 \\
\hline C20-C15-C16 & $118.28(12)$ & C20-C15-C14 & $121.28(12)$ \\
\hline C16-C15-C14 & $120.43(11)$ & C17-C16-C15 & $120.91(13)$ \\
\hline C17-C16-H16 & 119.5 & $\mathrm{C} 15-\mathrm{C} 16-\mathrm{H} 16$ & 119.5 \\
\hline C16-C17-C18 & $120.21(14)$ & $\mathrm{C} 16-\mathrm{C} 17-\mathrm{H} 17$ & 119.9 \\
\hline $\mathrm{C} 18-\mathrm{C} 17-\mathrm{H} 17$ & 119.9 & C19-C18-C17 & $119.64(13)$ \\
\hline C19-C18-H18 & 120.2 & $\mathrm{C} 17-\mathrm{C} 18-\mathrm{H} 18$ & 120.2 \\
\hline C18-C19-C20 & $119.95(12)$ & C18-C19-H19 & 120.0 \\
\hline C20-C19-H19 & 120.0 & C15-C20-C19 & $121.00(13)$ \\
\hline C15-C20-H20 & 119.5 & C19-C20-H20 & 119.5 \\
\hline
\end{tabular}

\section{Table 6. Torsion angles $\left({ }^{\circ}\right)$ for 22.}

C4-O1-C1-C3
O1-C1-C2-F3
O1-C1-C2-F2
O1-C1-C2-F1
O1-C1-C3-F6
O1-C1-C3-F5
O1-C1-C3-F4
C1-O1-C4-O2

$-117.76(11)$

$-60.59(14)$

59.12(14)

179.91(10)

$66.71(14)$

$-53.78(14)$

$-173.99(10)$

41.89(13)
$\mathrm{C} 4-\mathrm{O} 1-\mathrm{C} 1-\mathrm{C} 2$

$\mathrm{C} 3-\mathrm{C} 1-\mathrm{C} 2-\mathrm{F} 3$

$\mathrm{C} 3-\mathrm{C} 1-\mathrm{C} 2-\mathrm{F} 2$

$\mathrm{C} 3-\mathrm{C} 1-\mathrm{C} 2-\mathrm{F} 1$

$\mathrm{C} 2-\mathrm{C} 1-\mathrm{C} 3-\mathrm{F} 6$

$\mathrm{C} 2-\mathrm{C} 1-\mathrm{C} 3-\mathrm{F} 5$

$\mathrm{C} 2-\mathrm{C} 1-\mathrm{C} 3-\mathrm{F} 4$

C1-O1-C4-C5
121.43(11)

$-178.44(11)$

$-58.73(14)$

$62.05(14)$

$-174.87(11)$

64.63(14)

$-55.57(14)$

$-76.93(13)$ 


\begin{tabular}{llll} 
C1-O1-C4-C14 & $158.86(10)$ & C9-O2-C4-O1 & \multicolumn{1}{l}{$155.02(10)$} \\
C9-O2-C4-C5 & $-85.99(12)$ & C9-O2-C4-C14 & $43.74(14)$ \\
O1-C4-C5-C6 & $-178.98(9)$ & O2-C4-C5-C6 & $65.68(13)$ \\
C14-C4-C5-C6 & $-61.94(14)$ & C4-C5-C6-C7 & $-62.65(14)$ \\
C5-C6-C7-C8 & $72.36(14)$ & C6-C7-C8-C13 & $123.39(13)$ \\
C6-C7-C8-C9 & $-58.98(15)$ & C4-O2-C9-C10 & $-116.46(12)$ \\
C4-O2-C9-C8 & $67.28(14)$ & C13-C8-C9-C10 & $2.86(17)$ \\
C7-C8-C9-C10 & $-174.87(11)$ & C13-C8-C9-O2 & $178.93(10)$ \\
C7-C8-C9-O2 & $1.20(17)$ & O2-C9-C10-C11 & $-177.76(11)$ \\
C8-C9-C10-C11 & $-1.55(19)$ & C9-C10-C11-C12 & $-0.6(2)$ \\
C10-C11-C12-C13 & $1.3(2)$ & C11-C12-C13-C8 & $0.09(19)$ \\
C9-C8-C13-C12 & $-2.12(18)$ & C7-C8-C13-C12 & $175.61(11)$ \\
O1-C4-C14-C15 & $55.09(13)$ & O2-C4-C14-C15 & $167.13(10)$ \\
C5-C4-C14-C15 & $-65.87(14)$ & C4-C14-C15-C20 & $96.92(14)$ \\
C4-C14-C15-C16 & $-84.08(15)$ & C20-C15-C16-C17 & $-0.2(2)$ \\
C14-C15-C16-C17 & $-179.25(13)$ & C15-C16-C17-C18 & $0.7(2)$ \\
C16-C17-C18-C19 & $-0.7(2)$ & C17-C18-C19-C20 & $0.3(2)$ \\
C16-C15-C20-C19 & $-0.2(2)$ & C14-C15-C20-C19 & $178.86(12)$ \\
C18-C19-C20-C15 & $0.1(2)$ & &
\end{tabular}

\section{Table 7. Anisotropic atomic displacement parameters $\left(\AA^{2}\right)$ for 22.}

The anisotropic atomic displacement factor exponent takes the form: $-2 \pi^{2}\left[h^{2} a^{* 2} U_{11}+\ldots+2 h k a^{*} b^{*} U_{12}\right]$

$\mathrm{U}_{11}$

$\begin{array}{llll}\text { F1 } & 0.0276(4) & 0.0230(4) & 0.0375(5) \\ \text { F2 } & 0.0622(6) & 0.0260(5) & 0.0351(5) \\ \text { F3 } & 0.0348(5) & 0.0193(4) & 0.0751(7) \\ \text { F4 } & 0.0165(4) & 0.0473(6) & 0.0496(6) \\ \text { F5 } & 0.0384(5) & 0.0699(7) & 0.0328(5) \\ \text { F6 } & 0.0419(6) & 0.0239(5) & 0.0823(8) \\ \text { O1 } & 0.0145(4) & 0.0205(5) & 0.0165(4) \\ \text { O2 } & 0.0140(4) & 0.0161(4) & 0.0218(4) \\ \text { C1 } & 0.0135(5) & 0.0174(6) & 0.0173(6) \\ \text { C2 } & 0.0189(6) & 0.0200(6) & 0.0241(6) \\ \text { C3 } & 0.0193(6) & 0.0239(7) & 0.0306(7) \\ \text { C4 } & 0.0141(5) & 0.0152(6) & 0.0175(6) \\ \text { C5 } & 0.0197(6) & 0.0155(6) & 0.0181(6) \\ \text { C6 } & 0.0187(6) & 0.0213(7) & 0.0223(6) \\ \text { C7 } & 0.0192(6) & 0.0231(7) & 0.0201(6) \\ \text { C8 } & 0.0163(6) & 0.0200(6) & 0.0173(6) \\ \text { C9 } & 0.0148(5) & 0.0159(6) & 0.0184(6) \\ \text { C10 } & 0.0236(6) & 0.0218(7) & 0.0235(6) \\ \text { C11 } & 0.0315(7) & 0.0184(7) & 0.0274(7) \\ \text { C12 } & 0.0276(7) & 0.0170(6) & 0.0284(7) \\ \text { C13 } & 0.0183(6) & 0.0236(7) & 0.0240(6) \\ \text { C14 } & 0.0168(6) & 0.0170(6) & 0.0172(6)\end{array}$

$\mathrm{U}_{23}$

$0.0051(4)$

$0.0195(4)$

$-0.0014(5)$

$0.0108(5)$

$0.0201(5)$

$0.0025(5)$

$0.0071(4)$

$0.0080(4)$

$0.0062(5)$

$0.0087(5)$

$0.0074(6)$

$0.0063(5)$

$0.0044(5)$

$0.0057(5)$

$0.0078(5)$

$0.0075(5)$

$0.0066(5)$

$0.0063(5)$

$0.0038(6)$

$0.0083(6)$

$0.0114(6)$

$0.0022(5)$
$\mathrm{U}_{13}$ $-0.0056(4)$ $0.0061(5)$

$0.0291(5)$

$0.0060(4)$

$0.0228(4)$

$0.0368(6)$

$0.0011(3)$

$0.0025(3)$

$0.0025(4)$

$0.0051(5)$

$0.0089(5)$

$0.0028(4)$

$0.0038(5)$

$0.0068(5)$

$0.0074(5)$

$0.0013(5)$

$0.0007(4)$

$0.0063(5)$

$0.0030(6)$

$-0.0015(6)$

$0.0025(5)$

$0.0011(4)$
$\mathrm{U}_{12}$ $-0.0048(4)$ $0.0031(4)$ $0.0040(4)$ $0.0113(4)$ $0.0216(5)$ $0.0101(4)$ $0.0008(3)$ $0.0043(3)$ $0.0020(5)$ $0.0024(5)$ $0.0050(5)$ $0.0033(5)$ $0.0052(5)$ $0.0078(5)$ $0.0055(5)$ $0.0054(5)$ $0.0032(5)$ $0.0090(5)$ $0.0091(6)$ $0.0009(5)$ $0.0019(5)$ $0.0041(5)$ 


\begin{tabular}{|c|c|c|c|c|c|c|}
\hline & $U_{11}$ & $\mathrm{U}_{22}$ & $\mathrm{U}_{33}$ & $\mathrm{U}_{23}$ & $\mathrm{U}_{13}$ & $U_{12}$ \\
\hline C15 & $0.0186(6)$ & $0.0185(6)$ & $0.0139(6)$ & $0.0005(5)$ & $-0.0008(4)$ & $0.0051(5)$ \\
\hline C16 & $0.0253(7)$ & $0.0394(8)$ & $0.0210(6)$ & $0.0117(6)$ & $0.0080(5)$ & $0.0172(6)$ \\
\hline C17 & $0.0344(8)$ & $0.0478(10)$ & $0.0246(7)$ & $0.0203(7)$ & $0.0134(6)$ & $0.0197(7)$ \\
\hline C18 & $0.0312(7)$ & $0.0329(8)$ & $0.0204(6)$ & $0.0107(6)$ & $0.0026(5)$ & $0.0149(6)$ \\
\hline C19 & $0.0184(6)$ & $0.0232(7)$ & $0.0241(7)$ & $0.0021(5)$ & $-0.0004(5)$ & $0.0079(5)$ \\
\hline $\mathrm{C} 20$ & $0.0165(6)$ & $0.0205(6)$ & $0.0203(6)$ & $0.0027(5)$ & $0.0011(5)$ & $0.0027(5)$ \\
\hline
\end{tabular}

\section{Table 8. Hydrogen atomic coordinates and isotropic atomic displacement parameters $\left(\AA^{2}\right)$ for 22.}

\begin{tabular}{|c|c|c|c|c|}
\hline & & & & \\
\hline $\mathrm{H} 1$ & 0.3473 & 0.2614 & 0.0967 & 0.02 \\
\hline $\mathrm{H} 5 \mathrm{~A}$ & 0.5971 & 0.2410 & 0.0644 & 0.021 \\
\hline H5B & 0.7132 & 0.2133 & 0.1752 & 0.021 \\
\hline $\mathrm{H} 6 \mathrm{~A}$ & 0.8573 & 0.3223 & 0.0546 & 0.024 \\
\hline $\mathrm{H} 6 \mathrm{~B}$ & 0.8994 & 0.4382 & 0.1858 & 0.024 \\
\hline $\mathrm{H} 7 \mathrm{~A}$ & 0.8643 & 0.5466 & 0.0107 & 0.024 \\
\hline H7B & 0.6845 & 0.4601 & -0.0226 & 0.024 \\
\hline $\mathrm{H} 10$ & 0.5188 & 0.7231 & 0.2973 & 0.027 \\
\hline $\mathrm{H} 11$ & 0.6628 & 0.9676 & 0.3128 & 0.032 \\
\hline $\mathrm{H} 12$ & 0.8659 & 1.0061 & 0.2178 & 0.031 \\
\hline $\mathrm{H} 13$ & 0.9195 & 0.8033 & 0.1031 & 0.027 \\
\hline $\mathrm{H} 14 \mathrm{~A}$ & 0.8132 & 0.5209 & 0.3513 & 0.022 \\
\hline $\mathrm{H} 14 \mathrm{~B}$ & 0.6676 & 0.5138 & 0.4086 & 0.022 \\
\hline H16 & 0.5771 & 0.3006 & 0.4987 & 0.031 \\
\hline $\mathrm{H} 17$ & 0.6501 & 0.1363 & 0.6093 & 0.038 \\
\hline $\mathrm{H} 18$ & 0.8866 & 0.0835 & 0.6123 & 0.033 \\
\hline $\mathrm{H} 19$ & 1.0472 & 0.1921 & 0.5002 & 0.028 \\
\hline $\mathrm{H} 2 \mathrm{O}$ & 0.9727 & 0.3549 & 0.3874 & 0.025 \\
\hline
\end{tabular}




\section{Crystal Structure Report for 18}
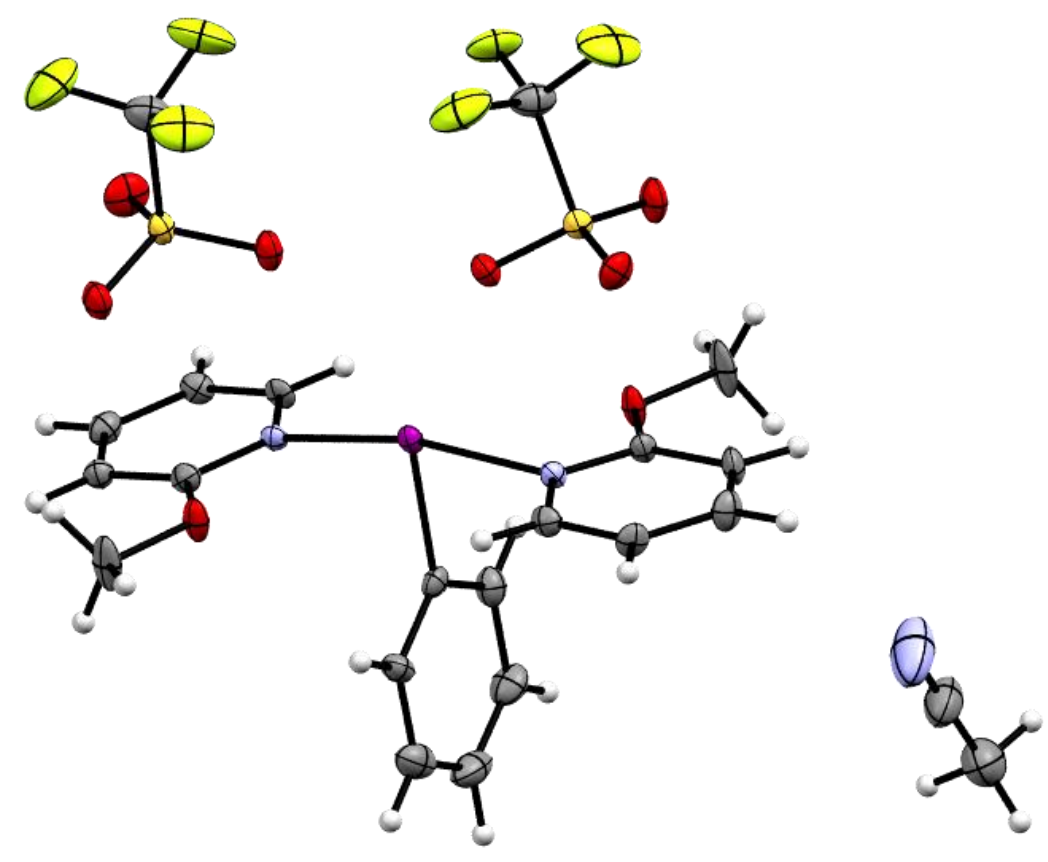

Figure 2. Crystal structure of $\left(\mathrm{C}_{18} \mathrm{H}_{19} \mathrm{~N}_{2} \mathrm{O}_{2} \mathrm{l}\right)\left(\mathrm{CF}_{3} \mathrm{SO}_{3}\right)_{2} \cdot\left(\mathrm{CH}_{3} \mathrm{CN}\right)$. Ellipsoids shown at $30 \%$ probability.

A specimen of $\mathrm{C}_{22} \mathrm{H}_{22} \mathrm{~F}_{6} \mathrm{IN}_{3} \mathrm{O}_{8} \mathrm{~S}_{2}$, approximate dimensions $0.020 \mathrm{~mm} \times 0.094 \mathrm{~mm} \times 0.216 \mathrm{~mm}$, was used for the $\mathrm{X}$-ray crystallographic analysis. The $\mathrm{X}$-ray intensity data were measured.

The integration of the data using a triclinic unit cell yielded a total of 7030 reflections to a maximum $\theta$ angle of $27.89^{\circ}(0.76 \AA$ A resolution), of which 7030 were independent (average redundancy 1.000 , completeness $=$ $\left.99.8 \%, R_{\text {sig }}=4.59 \%\right)$ and $5904(83.98 \%)$ were greater than $2 \sigma\left(\mathrm{F}^{2}\right)$. The final cell constants of $\mathrm{a}=$

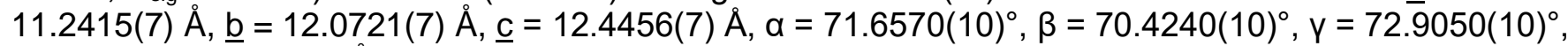
volume $=1476.55(15) \AA^{3}$, are based upon the refinement of the XYZ-centroids of reflections above $20 \sigma(\mathrm{I})$. The calculated minimum and maximum transmission coefficients (based on crystal size) are 0.6931 and 0.7456 .

The structure was solved and refined using the Bruker SHELXTL Software Package, using the space group $P-1$, with $Z=2$ for the formula unit, $\mathrm{C}_{22} \mathrm{H}_{22} \mathrm{~F}_{6} \mathrm{IN}_{3} \mathrm{O}_{8} \mathrm{~S}_{2}$. The final anisotropic full-matrix least-squares refinement on $\mathrm{F}^{2}$ with 383 variables converged at $\mathrm{R} 1=2.99 \%$, for the observed data and $\mathrm{wR} 2=6.83 \%$ for all data. The goodness-of-fit was 1.043. The largest peak in the final difference electron density synthesis was $1.019 \mathrm{e}^{-} / \AA^{3}$ and the largest hole was $-0.480 \mathrm{e}^{-} / \AA^{3}$ with an RMS deviation of $0.103 \mathrm{e}^{-} / \AA^{3}$. On the basis of the final model, the calculated density was $1.713 \mathrm{~g} / \mathrm{cm}^{3}$ and $F(000), 756 \mathrm{e}^{-}$.

\section{Table 9. Sample and crystal data for 18.}

\section{Identification code \\ Chemical formula \\ Formula weight \\ Temperature \\ Wavelength \\ Crystal size \\ Crystal system \\ Space group \\ Unit cell dimensions}

Volume
2894

$\mathrm{C}_{22} \mathrm{H}_{22} \mathrm{~F}_{6} \mathrm{IN}_{3} \mathrm{O}_{8} \mathrm{~S}_{2}$

$761.44 \mathrm{~g} / \mathrm{mol}$

$100(2) \mathrm{K}$

$0.71073 \AA$

$0.020 \times 0.094 \times 0.216 \mathrm{~mm}$

triclinic

$\mathrm{P}-1$

$\mathrm{a}=11.2415(7) \AA$

$\mathrm{b}=12.0721(7) \AA$

$c=12.4456(7) \AA$

1476.55(15) $\AA^{3}$ $\alpha=71.6570(10)^{\circ}$

$\beta=70.4240(10)^{\circ}$

$Y=72.9050(10)^{\circ}$ 
Density (calculated)

Absorption coefficient

$\mathbf{F}(000)$
$1.713 \mathrm{~g} / \mathrm{cm}^{3}$

$1.312 \mathrm{~mm}^{-1}$

756

\section{Table 10. Data collection and structure refinement for 18.}

Theta range for data collection

Reflections collected

Max. and min. transmission

Structure solution technique

Structure solution program

Refinement method

Refinement program

Function minimized

Data / restraints / parameters

Goodness-of-fit on $\mathrm{F}^{2}$

$\Delta / \sigma_{\max }$

Final $\mathbf{R}$ indices

Weighting scheme

Largest diff. peak and hole

R.M.S. deviation from mean
1.79 to $27.89^{\circ}$

7030

0.7456 and 0.6931

direct methods

SHELXS-97 (Sheldrick, 2008)

Full-matrix least-squares on $\mathrm{F}^{2}$

SHELXL-2014/7 (Sheldrick, 2014)

$\Sigma w\left(F_{o}^{2}-F_{c}^{2}\right)^{2}$

7030 / 0 / 383

1.043

0.002

5904 data; $\mathrm{I}>2 \sigma(\mathrm{I}) \quad \mathrm{R} 1=0.0299, \mathrm{wR} 2=0.0652$

all data

$\mathrm{R} 1=0.0433, \mathrm{wR} 2=0.0683$ $w=1 /\left[\sigma^{2}\left(F_{o}^{2}\right)+(0.0372 P)^{2}\right]$

where $\mathrm{P}=\left(\mathrm{F}_{\mathrm{o}}{ }^{2}+2 \mathrm{~F}_{\mathrm{c}}{ }^{2}\right) / 3$

1.019 and $-0.480 \mathrm{e}^{-3}$

$0.103 \mathrm{e}^{-3}$

\section{Table 11. Atomic coordinates and equivalent isotropic atomic displacement parameters $\left(\AA^{2}\right)$ for 18 .}

$\mathrm{U}(\mathrm{eq})$ is defined as one third of the trace of the orthogonalized $\mathrm{U}_{\mathrm{ij}}$ tensor.

\begin{tabular}{lllll} 
& \multicolumn{1}{c}{$\mathbf{x / a}$} & \multicolumn{1}{c}{$\mathbf{y} / \mathbf{b}$} & \multicolumn{1}{c}{$\mathbf{z} / \mathbf{c}$} & \multicolumn{1}{c}{$\mathbf{U}(\mathbf{e q})$} \\
I1 & $0.47593(2)$ & $0.60529(2)$ & $0.72543(2)$ & $0.01265(5)$ \\
N1 & $0.6094(3)$ & $0.6275(3)$ & $0.5456(3)$ & $0.0128(7)$ \\
N2 & $0.3327(3)$ & $0.6268(3)$ & $0.8956(3)$ & $0.0132(7)$ \\
N3 & $0.9209(5)$ & $0.8608(4)$ & $0.1554(5)$ & $0.0680(14)$ \\
S1 & $0.77172(10)$ & $0.32436(10)$ & $0.59240(8)$ & $0.0158(2)$ \\
S2 & $0.31220(10)$ & $0.32458(10)$ & $0.98632(9)$ & $0.0150(2)$ \\
O1 & $0.7525(2)$ & $0.6364(3)$ & $0.6276(2)$ & $0.0210(7)$ \\
O2 & $0.1857(2)$ & $0.6367(3)$ & $0.8095(2)$ & $0.0186(7)$ \\
O3 & $0.8728(2)$ & $0.3620(3)$ & $0.6098(2)$ & $0.0230(7)$ \\
O4 & $0.7793(3)$ & $0.3324(4)$ & $0.4725(3)$ & $0.0294(10)$ \\
O5 & $0.6440(2)$ & $0.3659(2)$ & $0.66199(19)$ & $0.0211(6)$ \\
O6 & $0.4153(2)$ & $0.3751(2)$ & $0.89522(19)$ & $0.0182(5)$ \\
O7 & $0.1931(2)$ & $0.3519(3)$ & $0.9548(2)$ & $0.0250(7)$ \\
O8 & $0.3000(3)$ & $0.3347(3)$ & $0.1028(2)$ & $0.0229(9)$ \\
F1 & $0.9220(3)$ & $0.1117(3)$ & $0.6025(3)$ & $0.0548(8)$ \\
F2 & $0.7948(3)$ & $0.1388(2)$ & $0.7696(2)$ & $0.0448(7)$ \\
F3 & $0.7200(3)$ & $0.1130(2)$ & $0.6436(2)$ & $0.0510(7)$
\end{tabular}




\begin{tabular}{|c|c|c|c|c|}
\hline & $\mathbf{x} / \mathbf{a}$ & $y / b$ & $\mathrm{z} / \mathrm{C}$ & $\mathrm{U}(\mathrm{eq}$ \\
\hline F4 & $0.4832(2)$ & $0.1271(2)$ & $0.0236(2)$ & $0.0457(7)$ \\
\hline F5 & $0.3889(2)$ & $0.1393(2)$ & $0.89421(18)$ & $0.0355(6)$ \\
\hline F6 & $0.2884(3)$ & $0.1024(2)$ & $0.0784(2)$ & $0.0544(8)$ \\
\hline $\mathrm{C} 1$ & $0.5678(3)$ & $0.6294(4)$ & $0.4541(3)$ & $0.0144(8)$ \\
\hline $\mathrm{C} 2$ & $0.6481(4)$ & $0.6387(4)$ & $0.3440(3)$ & $0.0173(9)$ \\
\hline C3 & $0.7754(4)$ & $0.6461(4)$ & $0.3257(4)$ & $0.0201(10)$ \\
\hline $\mathrm{C} 4$ & $0.8177(4)$ & $0.6453(4)$ & $0.4182(3)$ & $0.0164(10)$ \\
\hline C5 & $0.7314(4)$ & $0.6361(4)$ & $0.5291(3)$ & $0.0168(9)$ \\
\hline C6 & $0.8806(3)$ & $0.6399(5)$ & $0.6260(3)$ & $0.0302(11)$ \\
\hline $\mathrm{C7}$ & $0.3749(4)$ & $0.6279(4)$ & $0.9853(3)$ & $0.0171(8)$ \\
\hline $\mathrm{C} 8$ & $0.2902(4)$ & $0.6359(4)$ & $0.0929(3)$ & $0.0174(10)$ \\
\hline $\mathrm{C9}$ & $0.1594(4)$ & $0.6426(5)$ & $0.1077(4)$ & $0.0210(11)$ \\
\hline C10 & $0.1168(4)$ & $0.6442(4)$ & $0.0159(4)$ & $0.0197(11)$ \\
\hline C11 & $0.2079(4)$ & $0.6354(4)$ & $0.9084(3)$ & $0.0148(8)$ \\
\hline $\mathrm{C} 12$ & $0.0567(3)$ & $0.6388(5)$ & $0.8109(3)$ & $0.0317(12)$ \\
\hline C13 & $0.4358(4)$ & $0.7920(2)$ & $0.6822(3)$ & $0.0153(5)$ \\
\hline C14 & $0.3387(3)$ & $0.8519(4)$ & $0.6272(3)$ & $0.0222(9)$ \\
\hline C15 & $0.3109(4)$ & $0.9781(4)$ & $0.6024(3)$ & $0.0270(9)$ \\
\hline C16 & $0.3793(5)$ & $0.0352(3)$ & $0.6319(4)$ & $0.0322(8)$ \\
\hline C17 & $0.4767(4)$ & $0.9709(4)$ & $0.6884(3)$ & $0.0286(9)$ \\
\hline C18 & $0.5048(3)$ & $0.8497(4)$ & $0.7138(3)$ & $0.0187(8)$ \\
\hline C19 & $0.8041(4)$ & $0.1643(4)$ & $0.6543(3)$ & $0.0308(9)$ \\
\hline $\mathrm{C} 20$ & $0.3713(4)$ & $0.1653(4)$ & $0.9957(3)$ & $0.0274(9)$ \\
\hline $\mathrm{C} 21$ & $0.8659(4)$ & $0.9510(4)$ & $0.1127(4)$ & $0.0435(11)$ \\
\hline $\mathrm{C} 22$ & $0.7944(4)$ & $0.0631(4)$ & $0.0576(4)$ & $0.0451(12)$ \\
\hline
\end{tabular}

\section{Table 12. Bond lengths (Å) for 18.}

$\begin{array}{ll}\text { I1-C13 } & 2.091(3) \\ \text { I1-N2 } & 2.227(3) \\ \text { N1-C1 } & 1.361(4) \\ \text { N2-C7 } & 1.356(4) \\ \text { S1-O4 } & 1.440(3) \\ \text { S1-O5 } & 1.443(2) \\ \text { S2-O7 } & 1.438(2) \\ \text { S2-O8 } & 1.450(3) \\ \text { O1-C5 } & 1.325(4) \\ \text { O2-C11 } & 1.330(4) \\ \text { F1-C19 } & 1.328(5) \\ \text { F3-C19 } & 1.333(4) \\ \text { F5-C20 } & 1.333(4) \\ \text { C1-C2 } & 1.356(5) \\ \text { C2-C3 } & 1.397(5) \\ \text { C3-C4 } & 1.382(5) \\ \text { C4-C5 } & 1.388(5) \\ \text { C6-H6A } & 0.98 \\ \text { C6-H6C } & 0.98 \\ \text { C7-H7 } & 0.95\end{array}$

$\begin{array}{ll}\text { I1-N1 } & 2.223(3) \\ \text { N1-C5 } & 1.348(5) \\ \text { N2-C11 } & 1.334(5) \\ \text { N3-C21 } & 1.145(5) \\ \text { S1-O3 } & 1.440(3) \\ \text { S1-C19 } & 1.816(4) \\ \text { S2-O6 } & 1.449(2) \\ \text { S2-C20 } & 1.820(4) \\ \text { O1-C6 } & 1.446(4) \\ \text { O2-C12 } & 1.438(4) \\ \text { F2-C19 } & 1.343(4) \\ \text { F4-C20 } & 1.329(4) \\ \text { F6-C20 } & 1.337(4) \\ \text { C1-H1 } & 0.95 \\ \text { C2-H2 } & 0.95 \\ \text { C3-H3 } & 0.95 \\ \text { C4-H4 } & 0.95 \\ \text { C6-H6B } & 0.98 \\ \text { C7-C8 } & 1.372(5) \\ \text { C8-C9 } & 1.400(5)\end{array}$




$\begin{array}{llll}\mathrm{C} 8-\mathrm{H} 8 & 0.95 & \mathrm{C}-\mathrm{C} 10 & 1.372(5) \\ \mathrm{C} 9-\mathrm{H} 9 & 0.95 & \mathrm{C} 10-\mathrm{C} 11 & 1.398(5) \\ \mathrm{C} 10-\mathrm{H} 10 & 0.95 & \mathrm{C} 12-\mathrm{H} 12 \mathrm{~A} & 0.98 \\ \mathrm{C} 12-\mathrm{H} 12 \mathrm{~B} & 0.98 & \mathrm{C} 12-\mathrm{H} 12 \mathrm{C} & 0.98 \\ \mathrm{C} 13-\mathrm{C} 14 & 1.380(5) & \mathrm{C} 13-\mathrm{C} 18 & 1.392(5) \\ \mathrm{C} 14-\mathrm{C} 15 & 1.415(6) & \mathrm{C} 14-\mathrm{H} 14 & 0.95 \\ \mathrm{C} 15-\mathrm{C} 16 & 1.365(6) & \mathrm{C} 15-\mathrm{H} 15 & 0.95 \\ \mathrm{C} 16-\mathrm{C} 17 & 1.403(6) & \mathrm{C} 16-\mathrm{H} 16 & 0.95 \\ \mathrm{C} 17-\mathrm{C} 18 & 1.358(6) & \mathrm{C} 17-\mathrm{H} 17 & 0.95 \\ \mathrm{C} 18-\mathrm{H} 18 & 0.95 & \mathrm{C} 21-\mathrm{C} 22 & 1.446(6) \\ \mathrm{C} 22-\mathrm{H} 22 \mathrm{~A} & 0.98 & \mathrm{C} 22-\mathrm{H} 22 \mathrm{~B} & 0.98 \\ \mathrm{C} 22-\mathrm{H} 22 \mathrm{C} & 0.98 & & \end{array}$

\section{Table 13. Bond angles $\left({ }^{\circ}\right)$ for 18.}

\begin{tabular}{|c|c|c|c|}
\hline C13-I1-N1 & $83.52(13)$ & C13-I1-N2 & $83.85(13)$ \\
\hline $\mathrm{N} 1-11-\mathrm{N} 2$ & $167.37(8)$ & C5-N1-C1 & $120.8(3)$ \\
\hline C5-N1-I1 & 119.6(3) & C1-N1-I1 & 119.6(2) \\
\hline C11-N2-C7 & $121.3(3)$ & C11-N2-I1 & 119.6(3) \\
\hline C7-N2-I1 & $119.1(2)$ & $\mathrm{O} 4-\mathrm{S} 1-\mathrm{O} 3$ & $115.93(17)$ \\
\hline O4-S1-O5 & $114.49(17)$ & O3-S1-O5 & $114.37(15)$ \\
\hline O4-S1-C19 & 102.7(2) & O3-S1-C19 & $103.16(19)$ \\
\hline O5-S1-C19 & $103.83(18)$ & O7-S2-O6 & $114.67(16)$ \\
\hline O7-S2-O8 & $115.70(15)$ & O6-S2-O8 & $114.17(17)$ \\
\hline O7-S2-C20 & 104.09(19) & O6-S2-C20 & $102.48(17)$ \\
\hline O8-S2-C20 & 103.4(2) & C5-O1-C6 & $118.9(3)$ \\
\hline C11-O2-C12 & $118.7(3)$ & $\mathrm{C} 2-\mathrm{C} 1-\mathrm{N} 1$ & $121.1(3)$ \\
\hline $\mathrm{C} 2-\mathrm{C} 1-\mathrm{H} 1$ & 119.5 & $\mathrm{~N} 1-\mathrm{C} 1-\mathrm{H} 1$ & 119.5 \\
\hline C1-C2-C3 & $118.8(4)$ & $\mathrm{C} 1-\mathrm{C} 2-\mathrm{H} 2$ & 120.6 \\
\hline C3-C2-H2 & 120.6 & C4-C3-C2 & $120.4(4)$ \\
\hline $\mathrm{C} 4-\mathrm{C} 3-\mathrm{H} 3$ & 119.8 & $\mathrm{C} 2-\mathrm{C} 3-\mathrm{H} 3$ & 119.8 \\
\hline C3-C4-C5 & $118.5(4)$ & C3-C4-H4 & 120.7 \\
\hline C5-C4-H4 & 120.7 & O1-C5-N1 & $112.0(3)$ \\
\hline O1-C5-C4 & $127.6(4)$ & $\mathrm{N} 1-\mathrm{C} 5-\mathrm{C} 4$ & $120.4(4)$ \\
\hline $\mathrm{O} 1-\mathrm{C} 6-\mathrm{H} 6 \mathrm{~A}$ & 109.5 & O1-C6-H6B & 109.5 \\
\hline H6A-C6-H6B & 109.5 & $\mathrm{O} 1-\mathrm{C} 6-\mathrm{H} 6 \mathrm{C}$ & 109.5 \\
\hline H6A-C6-H6C & 109.5 & H6B-C6-H6C & 109.5 \\
\hline N2-C7-C8 & $120.7(3)$ & $\mathrm{N} 2-\mathrm{C} 7-\mathrm{H} 7$ & 119.6 \\
\hline $\mathrm{C} 8-\mathrm{C} 7-\mathrm{H} 7$ & 119.6 & C7-C8-C9 & $118.3(4)$ \\
\hline $\mathrm{C} 7-\mathrm{C} 8-\mathrm{H} 8$ & 120.8 & C9-C8-H8 & 120.8 \\
\hline C10-C9-C8 & $120.7(4)$ & C10-C9-H9 & 119.6 \\
\hline C8-C9-H9 & 119.6 & C9-C10-C11 & $118.3(4)$ \\
\hline $\mathrm{C} 9-\mathrm{C} 10-\mathrm{H} 10$ & 120.9 & $\mathrm{C} 11-\mathrm{C} 10-\mathrm{H} 10$ & 120.9 \\
\hline O2-C11-N2 & $112.1(3)$ & $\mathrm{O} 2-\mathrm{C} 11-\mathrm{C} 10$ & $127.3(4)$ \\
\hline $\mathrm{N} 2-\mathrm{C} 11-\mathrm{C} 10$ & $120.7(3)$ & $\mathrm{O} 2-\mathrm{C} 12-\mathrm{H} 12 \mathrm{~A}$ & 109.5 \\
\hline $\mathrm{O} 2-\mathrm{C} 12-\mathrm{H} 12 \mathrm{~B}$ & 109.5 & $\mathrm{H} 12 \mathrm{~A}-\mathrm{C} 12-\mathrm{H} 12 \mathrm{~B}$ & 109.5 \\
\hline $\mathrm{O} 2-\mathrm{C} 12-\mathrm{H} 12 \mathrm{C}$ & 109.5 & $\mathrm{H} 12 \mathrm{~A}-\mathrm{C} 12-\mathrm{H} 12 \mathrm{C}$ & 109.5 \\
\hline $\mathrm{H} 12 \mathrm{~B}-\mathrm{C} 12-\mathrm{H} 12 \mathrm{C}$ & 109.5 & C14-C13-C18 & $123.1(3)$ \\
\hline C14-C13-I1 & $118.2(3)$ & C18-C13-I1 & $118.6(3)$ \\
\hline
\end{tabular}




$\begin{array}{llll}\text { C13-C14-C15 } & 117.0(3) & \mathrm{C} 13-\mathrm{C} 14-\mathrm{H} 14 & 121.5 \\ \mathrm{C} 15-\mathrm{C} 14-\mathrm{H} 14 & 121.5 & \mathrm{C} 16-\mathrm{C} 15-\mathrm{C} 14 & 120.1(3) \\ \mathrm{C} 16-\mathrm{C} 15-\mathrm{H} 15 & 120.0 & \mathrm{C} 14-\mathrm{C} 15-\mathrm{H} 15 & 120.0 \\ \mathrm{C} 15-\mathrm{C} 16-\mathrm{C} 17 & 121.1(3) & \mathrm{C} 15-\mathrm{C} 16-\mathrm{H} 16 & 119.4 \\ \mathrm{C} 17-\mathrm{C} 16-\mathrm{H} 16 & 119.4 & \mathrm{C} 18-\mathrm{C} 17-\mathrm{C} 16 & 119.9(4) \\ \mathrm{C} 18-\mathrm{C} 17-\mathrm{H} 17 & 120.0 & \mathrm{C} 16-\mathrm{C} 17-\mathrm{H} 17 & 120.0 \\ \mathrm{C} 17-\mathrm{C} 18-\mathrm{C} 13 & 118.7(3) & \mathrm{C} 17-\mathrm{C} 18-\mathrm{H} 18 & 120.6 \\ \mathrm{C} 13-\mathrm{C} 18-\mathrm{H} 18 & 120.6 & \mathrm{~F} 1-\mathrm{C} 19-\mathrm{F} 3 & 107.8(4) \\ \text { F1-C19-F2 } & 107.4(3) & \mathrm{F} 3-\mathrm{C} 19-\mathrm{F} 2 & 106.7(3) \\ \text { F1-C19-S1 } & 111.7(3) & \mathrm{F} 3-\mathrm{C} 19-\mathrm{S} 1 & 111.6(3) \\ \text { F2-C19-S1 } & 111.4(3) & \mathrm{F} 4-\mathrm{C} 20-\mathrm{F} 5 & 108.0(3) \\ \text { F4-C20-F6 } & 107.2(3) & \mathrm{F} 5-\mathrm{C} 20-\mathrm{F} 6 & 107.7(3) \\ \text { F4-C20-S2 } & 110.8(3) & \mathrm{F} 5-\mathrm{C} 20-\mathrm{S} 2 & 111.6(3) \\ \text { F6-C20-S2 } & 111.2(3) & \mathrm{N} 3-\mathrm{C} 21-\mathrm{C} 22 & 178.3(6) \\ \text { C21-C22-H22A } & 109.5 & \mathrm{C} 21-\mathrm{C} 22-\mathrm{H} 22 \mathrm{~B} & 109.5 \\ \mathrm{H} 22 \mathrm{~A}-\mathrm{C} 22-\mathrm{H} 22 \mathrm{~B} & 109.5 & \mathrm{C} 21-\mathrm{C} 22-\mathrm{H} 22 \mathrm{C} & 109.5 \\ \mathrm{H} 22 \mathrm{~A}-\mathrm{C} 22-\mathrm{H} 22 \mathrm{C} & 109.5 & \mathrm{H} 22 \mathrm{~B}-\mathrm{C} 22-\mathrm{H} 22 \mathrm{C} & 109.5\end{array}$

\section{Table 14. Torsion angles $\left({ }^{\circ}\right)$ for 18.}
C5-N1-C1-C2
$\mathrm{N} 1-\mathrm{C} 1-\mathrm{C} 2-\mathrm{C} 3$
$\mathrm{C} 2-\mathrm{C} 3-\mathrm{C} 4-\mathrm{C} 5$
C6-O1-C5-C4
I1-N1-C5-O1
I1-N1-C5-C4
C3-C4-C5-N1
I1-N2-C7-C8
$\mathrm{C} 7-\mathrm{C} 8-\mathrm{C} 9-\mathrm{C} 10$
C12-O2-C11-N2
C7-N2-C11-O2
C7-N2-C11-C10
$\mathrm{C} 9-\mathrm{C} 10-\mathrm{C} 11-\mathrm{O} 2$
C18-C13-C14-C15
C13-C14-C15-C16
C15-C16-C17-C18
C14-C13-C18-C17
O4-S1-C19-F1
O5-S1-C19-F1
O3-S1-C19-F3
O4-S1-C19-F2
O5-S1-C19-F2
O6-S2-C20-F4
O7-S2-C20-F5
O8-S2-C20-F5
O6-S2-C20-F6

$0.7(6)$

$0.1(6)$

$0.5(7)$

$-3.8(7)$

$-3.6(5)$

177.5(3)

$0.3(7)$

$-177.2(3)$

$-1.6(7)$

176.5(4)

177.9(4)

$-0.9(6)$

$-179.2(4)$

$0.4(6)$

$0.4(5)$

$0.5(6)$

$-0.8(6)$

$62.9(3)$

$-177.5(3)$

$-178.7(3)$

$-176.9(3)$

$-57.4(3)$

$59.7(3)$

$59.0(3)$

$-179.7(3)$

178.9(3)
I1-N1-C1-C2

C1-C2-C3-C4

C6-O1-C5-N1

C1-N1-C5-O1

C1-N1-C5-C4

C3-C4-C5-O1

C11-N2-C7-C8

N2-C7-C8-C9

C8-C9-C10-C11

C12-O2-C11-C10

I1-N2-C11-O2

I1-N2-C11-C10

C9-C10-C11-N2

I1-C13-C14-C15

C14-C15-C16-C17

C16-C17-C18-C13

I1-C13-C18-C17

O3-S1-C19-F1

O4-S1-C19-F3

O5-S1-C19-F3

O3-S1-C19-F2

O7-S2-C20-F4

O8-S2-C20-F4

O6-S2-C20-F5

O7-S2-C20-F6

O8-S2-C20-F6
$-177.7(3)$

$-0.7(7)$

$177.4(4)$

$178.0(4)$

$-1.0(6)$

$-178.4(4)$

$1.1(6)$

$0.1(6)$

$1.8(7)$

$-4.8(7)$

$-3.8(5)$

177.4(4)

$-0.6(7)$

178.0(3)

$-0.9(6)$

$0.3(5)$

$-178.4(3)$

$-57.9(3)$

$-57.8(3)$

$61.7(3)$

62.2(3)

$179.4(3)$

$-59.3(3)$

$-60.8(3)$

$-61.4(3)$

$59.9(3)$ 


\section{Table 15. Anisotropic atomic displacement parameters $\left(\AA^{2}\right)$ for 18.}

The anisotropic atomic displacement factor exponent takes the form: $-2 \pi^{2}\left[h^{2} a^{* 2} U_{11}+\ldots+2 h k a^{*} b^{*} U_{12}\right]$

\begin{tabular}{|c|c|c|c|c|c|c|}
\hline & $\mathrm{U}_{11}$ & $\mathbf{U}_{22}$ & $\mathbf{U}_{33}$ & $\mathbf{U}_{23}$ & $\mathbf{U}_{13}$ & $\mathbf{U}_{12}$ \\
\hline 11 & $0.01188(9)$ & $0.01701(9)$ & $0.01056(8)$ & $-0.00365(9)$ & $-0.00291(6)$ & $-0.00495(10)$ \\
\hline $\mathrm{N} 1$ & $0.0124(17)$ & $0.0155(19)$ & $0.0133(15)$ & $-0.0053(14)$ & $-0.0040(14)$ & $-0.0042(14)$ \\
\hline N2 & $0.0139(17)$ & $0.0163(19)$ & $0.0110(15)$ & $-0.0042(14)$ & $-0.0019(14)$ & $-0.0059(15)$ \\
\hline N3 & $0.039(2)$ & $0.052(3)$ & $0.073(3)$ & $0.006(3)$ & $0.002(2)$ & $0.007(3)$ \\
\hline S1 & $0.0148(5)$ & $0.0218(6)$ & $0.0119(4)$ & $-0.0046(4)$ & $-0.0024(3)$ & $-0.0061(4)$ \\
\hline $\mathrm{S} 2$ & $0.0153(5)$ & $0.0187(6)$ & $0.0124(4)$ & $-0.0044(4)$ & $-0.0039(4)$ & $-0.0047(4)$ \\
\hline 01 & $0.0116(14)$ & $0.0400(19)$ & $0.0159(13)$ & $-0.0112(12)$ & $-0.0039(13)$ & $-0.0068(14)$ \\
\hline $\mathrm{O} 2$ & $0.0097(14)$ & $0.0366(19)$ & $0.0129(13)$ & $-0.0071(12)$ & $-0.0042(12)$ & $-0.0073(13)$ \\
\hline O3 & $0.0145(14)$ & $0.0309(19)$ & $0.0258(14)$ & $-0.0089(13)$ & $-0.0039(11)$ & $-0.0074(12)$ \\
\hline O4 & $0.041(2)$ & $0.036(2)$ & $0.0127(14)$ & $-0.0071(14)$ & $-0.0081(15)$ & $-0.0079(17)$ \\
\hline O5 & $0.0136(12)$ & $0.0329(16)$ & $0.0188(12)$ & $-0.0084(11)$ & $-0.0036(10)$ & $-0.0061(12)$ \\
\hline O6 & $0.0160(12)$ & $0.0221(14)$ & $0.0180(11)$ & $-0.0036(11)$ & $-0.0034(10)$ & $-0.0086(11)$ \\
\hline $\mathrm{O} 7$ & $0.0120(13)$ & $0.040(2)$ & $0.0251(14)$ & $-0.0107(13)$ & $-0.0056(11)$ & $-0.0045(13)$ \\
\hline O8 & $0.0255(17)$ & $0.028(2)$ & $0.0141(14)$ & $-0.0099(13)$ & $-0.0017(13)$ & $-0.0032(14)$ \\
\hline $\mathrm{F} 1$ & $0.0534(17)$ & $0.0332(16)$ & $0.0665(18)$ & $-0.0193(15)$ & $-0.0165(15)$ & $0.0157(13)$ \\
\hline $\mathrm{F} 2$ & $0.0725(18)$ & $0.0320(15)$ & $0.0308(13)$ & $0.0071(11)$ & $-0.0258(12)$ & $-0.0140(13)$ \\
\hline F3 & $0.0763(19)$ & $0.0355(16)$ & $0.0596(17)$ & $-0.0033(13)$ & $-0.0321(15)$ & $-0.0309(15)$ \\
\hline $\mathrm{F} 4$ & $0.0585(17)$ & $0.0276(15)$ & $0.0580(16)$ & $-0.0164(12)$ & $-0.0401(14)$ & $0.0157(12)$ \\
\hline F5 & $0.0571(16)$ & $0.0274(14)$ & $0.0309(12)$ & $-0.0156(10)$ & $-0.0165(11)$ & $-0.0070(12)$ \\
\hline F6 & $0.088(2)$ & $0.0350(15)$ & $0.0347(13)$ & $0.0040(12)$ & $-0.0025(14)$ & $-0.0351(15)$ \\
\hline $\mathrm{C} 1$ & $0.0133(19)$ & $0.020(2)$ & $0.0153(18)$ & $-0.0047(16)$ & $-0.0081(15)$ & $-0.0058(16)$ \\
\hline $\mathrm{C} 2$ & $0.020(2)$ & $0.019(2)$ & $0.0150(19)$ & $-0.0025(17)$ & $-0.007(2)$ & $-0.0058(19)$ \\
\hline C3 & $0.022(2)$ & $0.020(3)$ & $0.0159(19)$ & $-0.0049(18)$ & $-0.0041(17)$ & $-0.0025(19)$ \\
\hline C4 & $0.017(2)$ & $0.018(2)$ & $0.0159(19)$ & $-0.0057(18)$ & $-0.0042(19)$ & $-0.0046(19)$ \\
\hline C5 & $0.015(2)$ & $0.018(2)$ & $0.0210(19)$ & $-0.0067(17)$ & $-0.0081(18)$ & $-0.0020(18)$ \\
\hline C6 & $0.013(2)$ & $0.064(3)$ & $0.0220(19)$ & $-0.017(2)$ & $-0.0067(17)$ & $-0.010(2)$ \\
\hline C7 & $0.016(2)$ & $0.018(2)$ & $0.0192(19)$ & $-0.0043(17)$ & $-0.0083(16)$ & $-0.0028(17)$ \\
\hline C8 & $0.020(2)$ & $0.024(2)$ & $0.0110(18)$ & $-0.0073(17)$ & $-0.0055(19)$ & $-0.0037(19)$ \\
\hline C9 & $0.020(2)$ & $0.030(3)$ & $0.0120(18)$ & $-0.0057(17)$ & $-0.0021(16)$ & $-0.004(2)$ \\
\hline C10 & $0.010(2)$ & $0.030(3)$ & $0.019(2)$ & $-0.0078(18)$ & $0.0010(19)$ & $-0.007(2)$ \\
\hline C11 & $0.014(2)$ & $0.020(2)$ & $0.0119(16)$ & $-0.0062(16)$ & $-0.0025(16)$ & $-0.0054(18)$ \\
\hline C12 & $0.011(2)$ & $0.066(4)$ & $0.025(2)$ & $-0.011(2)$ & $-0.0071(17)$ & $-0.013(2)$ \\
\hline C13 & $0.0148(14)$ & $0.0163(14)$ & $0.0115(13)$ & $-0.0046(16)$ & $-0.0006(10)$ & $-0.0008(18)$ \\
\hline C14 & $0.019(2)$ & $0.028(2)$ & $0.0175(17)$ & $-0.0046(17)$ & $-0.0032(15)$ & $-0.0051(18)$ \\
\hline C15 & $0.027(2)$ & $0.023(2)$ & $0.0247(18)$ & $-0.0031(16)$ & $-0.0086(16)$ & $0.0028(17)$ \\
\hline C16 & $0.041(2)$ & $0.0183(17)$ & $0.0272(19)$ & $-0.0064(19)$ & $0.0032(16)$ & $-0.004(2)$ \\
\hline C17 & $0.038(2)$ & $0.031(2)$ & $0.0188(17)$ & $-0.0091(16)$ & $0.0007(15)$ & $-0.0165(19)$ \\
\hline C18 & $0.023(2)$ & $0.019(2)$ & $0.0170(16)$ & $-0.0052(15)$ & $-0.0043(14)$ & $-0.0096(17)$ \\
\hline C19 & $0.040(2)$ & $0.027(2)$ & $0.029(2)$ & $-0.0050(18)$ & $-0.0134(18)$ & $-0.011(2)$ \\
\hline C20 & $0.041(2)$ & $0.022(2)$ & $0.0207(18)$ & $-0.0050(16)$ & $-0.0090(16)$ & $-0.0083(18)$ \\
\hline C21 & $0.032(2)$ & $0.033(2)$ & $0.047(3)$ & $-0.005(2)$ & $0.0019(18)$ & $0.000(2)$ \\
\hline C22 & $0.040(3)$ & $0.044(3)$ & $0.047(3)$ & $-0.011(2)$ & $-0.002(2)$ & $-0.013(2)$ \\
\hline
\end{tabular}




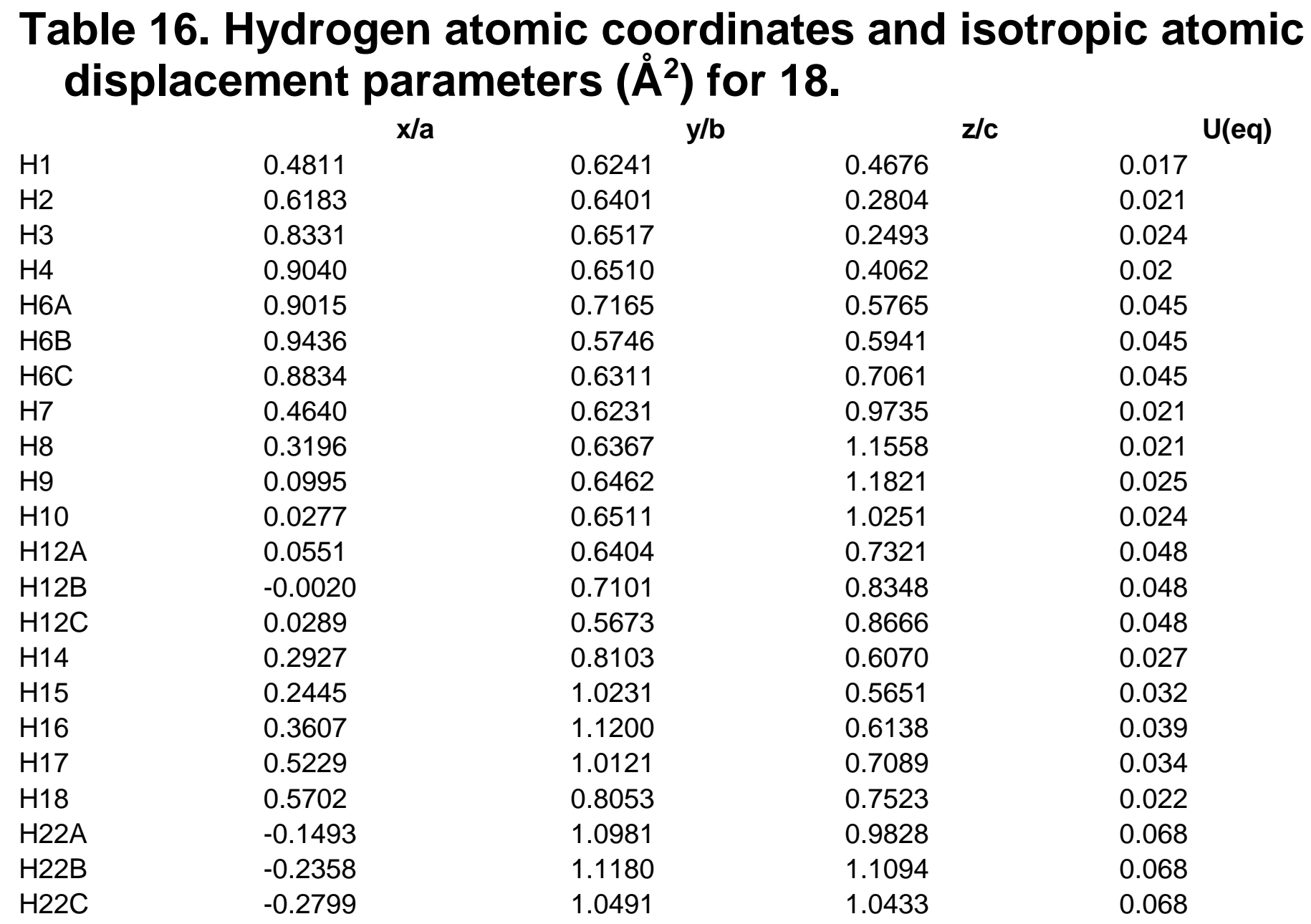

